\title{
Automorphisms of compact groups
}

\author{
BRUCE KITCHENS AND KLAUS SCHMIDT \\ Mathematical Sciences Department, IBM T. J. Watson Research Center, Yorktown \\ Heights, NY, USA \\ Mathematics Institute, University of Warwick, Coventry, UK
}

(Received 24 September 1987 and revised 9 August 1988)

\begin{abstract}
We study finitely generated, abelian groups $\Gamma$ of continuous automorphisms of a compact, metrizable group $X$ and introduce the descending chain condition for such pairs $(X, \Gamma)$. If $\Gamma$ acts expansively on $X$ then $(X, \Gamma)$ satisfies the descending chain condition, and $(X, \Gamma)$ satisfies the descending chain condition if and only if it is algebraically and topologically isomorphic to a closed, shift-invariant subgroup of $G^{\mathbf{r}}$, where $G$ is a compact Lie group. Furthermore every such subgroup of $G^{\Gamma}$ is a (higher dimensional) Markov shift whose alphabet is a compact Lie group. By using the descending chain condition we prove, for example, that the set of $\Gamma$-periodic points is dense in $X$ whenever $\Gamma$ acts expansively on $X$. Furthermore, if $X$ is a compact group and $(X, \Gamma)$ satisfies the descending chain condition, then every ergodic element of $\Gamma$ has a dense set of periodic points. Finally we give an algebraic description of pairs $(X, \Gamma)$ satisfying the descending chain condition under the assumption that $X$ is abelian.
\end{abstract}

\section{Introduction}

This paper is motivated by the following questions:

(i) Let $\Gamma$ be a finitely generated, abelian group of continuous automorphisms of a compact group $X$. When does $\Gamma$ have a dense set of periodic (i.e. finite) orbits in $X$ ?

(ii) If the group $\Gamma$ acts expansively on $X$, what are the algebraic and topological properties of the dynamical system $(X, \Gamma)$ ?

We show that expansiveness of $\Gamma$ implies a weaker notion, the descending chain condition on closed, $\Gamma$-invariant subgroups of $X$, which in turn has two remarkable consequences: the system $(X, \Gamma)$ is algebraically and topologically isomorphic to a (higher dimensional) subshift of finite type whose alphabet is a compact Lie group. Furthermore, if $G$ is a compact Lie group, then the closed, shift invariant subgroups of $G^{\prime}$ automatically satisfy the descending chain condition and present an interesting class of examples of higher dimensional Markov shifts which lack many of the pathologies traditionally associated with such shifts (cf. [Ro] and [Ru]).

The contents of this paper are organized as follows: in $\S 2$ we assume that $X$ is a (metrizable) compact group and $\Gamma$ a countable (and always infinite) group of continuous automorphisms of $X$, and discuss some general properties of the dynamical system $(X, \Gamma)$. Lemma 2.2 shows that $\Gamma$ is nonergodic if and only if there exists 
a closed, normal, $\Gamma$-invariant subgroup $V$ of $X$ such that the action of $\Gamma$ on the quotient group $X / V$ is equicontinuous. From this one concludes easily (a special case of) a result due to $D$. Berend that $(X, \Gamma)$ is ergodic (with respect to Haar measure on $X$ ) if and only if $\Gamma$ is topologically transitive on $X$ (Theorem 2.1). Lemma 2.2 allows us to analyze the structure of nonergodic pairs $(X, \Gamma)$ (cf. Theorem 2.3). Finally, $\Gamma$ is mixing if and only if every element $\gamma \in \Gamma$ of infinite order is ergodic (Theorem 2.4).

In $\S \S 3-12$ we assume that $\Gamma$ is a finitely generated, abelian group of automorphisms of a compact group $X$. Theorem 3.2 states that $(X, \Gamma)$ satisfies the descending chain condition if and only if there exists a compact Lie group $G$ such that $(X, \Gamma)$ is algebraically and topologically isomorphic to a closed, shift-invariant subgroup of $G^{\Gamma}$. Furthermore every closed, shift-invariant subgroup $Y \subset G^{\Gamma}$ is a subshift of finite type whose alphabet is a compact Lie group (Corollary 3.8$)$. If $(X, \Gamma)$ satisfies the descending chain condition and $\Gamma$ is nonergodic on $X$ then there exists a closed, normal, $\Gamma$-invariant subgroup $X^{\prime} \subset X$ such that $\Gamma$ is ergodic on $X^{\prime}$ and $X / X^{\prime}$ is a Lie group (Theorem 3.15). Theorem 3.16 shows that every pair $(X, \Gamma)$ is a projective limit of pairs $\left(X_{n}, \Gamma_{n}\right), n \geq 1$, satisfying the descending chain condition.

$\S 4$ contains some technical results which, under certain conditions, reduce the study of pairs $(X, \Gamma)$ satisfying the descending chain condition to that of closed, shift-invariant subgroups of $G^{r}$, where $G$ is a compact Lie group which is either finite or has finite centre. In particular, if the connected component $X^{0}$ of the identity in $X$ is abelian then there exists an increasing sequence $\left(X_{n}, n \geq 1\right)$ of closed, normal, zero dimensional, $\Gamma$-invariant subgroups of $X$ such that $\bigcup_{n} X_{n}$ is dense in $X$ (Corollary 4.8 ).

In $\S \S 5$ and 6 we analyze expansive automorphisms of compact groups. Theorem 5.2 shows that, if $X$ is a compact group and $\Gamma$ is expansive, then $(X, \Gamma)$ satisfies the descending chain condition. The structure of expansive automorphisms of compact groups is discussed in detail in $\$ 6$, where we extend and combine earlier results by Arov, Lawton, Miles, Thomas, and many others to obtain a complete description of such maps (Theorem 6.7).

$\$ 7$ again deals with periodic points. If $X$ is a compact group such that $X^{0}$ is abelian and $(X, \Gamma)$ satisfies the descending chain condition, then $\Gamma$ has a dense set of periodic points (Theorem 7.2). If $X$ is a compact group, and if $\Gamma \subset$ Aut $(X)$ acts expansively on $X$, then $X^{0}$ must be abelian (cf. [La]), and the set of $\Gamma$-periodic points is dense in $X$ (Theorem 7.3 and Corollary 7.4). Finally, if $X$ is a compact group, if $\alpha \in$ Aut $(X)$ is an ergodic (or, equivalently, topologically transitive) automorphism which commutes with $\Gamma \subset$ Aut $(X)$, and if $(X, \Gamma)$ satisfies the descending chain condition, then the set of periodic points of $\alpha$ is dense in $X$ (Theorem 7.5 and Corollary 7.6). Neither the descending chain condition nor the assumption of ergodicity of $\alpha$ can be removed in general (Examples 7.7). Furthermore, if $(X, \Gamma)$ satisfies the descending chain condition and $\Gamma$ is ergodic on $X$, the set of $\Gamma$-periodic points is - in general - not dense (Example 7.8).

$\S \S 8-10$ contain the proofs of the Theorems $7.2,7.3$ and 7.5 , respectively. Although Theorem 7.3 is a special case of Theorem 3.2 in [La] we include a proof based on 
the descending and ascending chain conditions which gives some insight into the dynamics of closed, shift invariant subgroups of $G^{\mathbf{I}}$, where $G$ is a compact, connected Lie group with trivial centre.

The last two sections (11 and 12) deal with the case where $X$ is abelian group and $(X, \Gamma)$ satisfies the descending chain condition. The main result in $\S 11$ (Theorem 11.4) states that such a dynamical system is determined by a finitely generated module over a ring of Laurent polynomials with integral coefficients in finitely many variables. The ergodicity and mixing properties of the dynamical system $(X, \Gamma)$ can be described in terms of these modules in a very straightforward manner (Theorem 11.2). The last section presents a number of examples.

This paper has benefited from conversations with a number of colleagues, and we would particularly like to acknowledge our indebtedness to Don Coppersmith (for alerting us to the connection between the dynamical systems in $\$ 11$ and polynomial rings), Brian Hartley (for allowing us to include his proof of Theorem 11.3), Mike Keane (for a number of discussions when we first got involved with these problems), François Ledrappier (for pointing out to us his paper [Le] and the examples therein), Doug Lind (for discussions about solenoids), and the referee (for alerting us to the reference [La]). There is some overlap between this paper and several of the earlier references: e.g. $\$ \S 5$ and 6 are closely related to the results in [MT] (where the descending chain condition is used implicitly to derive the Markov property for a single group automorphism), and Theorems 5.3 and 7.3 are strictly contained in Theorem 3.2 in [La] (where $\Gamma$ is allowed to be an arbitrary semigroup in Aut $(X)$ ). The reason for including some of these earlier results in this paper is to present a coherent picture of the theory of automorphisms of compact groups as (higher dimensional) Markov shifts, and to provide the basis for further analysis of automorphisms of compact, abelian groups in subsequent papers (cf. $[$ S2, S3, LSW]).

\section{Topological transitivity, ergodicity, and mixing}

If $X$ is a group the identity element of $X$ will usually be denoted by 1 (or $1_{X}$, if there is any danger of confusion). Throughout this paper the term compact group will denote a compact, second countable (i.e. metrizable) group, and a compact Lie group will be a (possibly finite) compact subgroup of some finite dimensional matrix group over the complex numbers. Let $X$ be a compact group. The centre of $X$ is denoted by $C(X)$, and $X^{0}$ stands for the connected component of the identity in $X$. Any metric $d$ on $X$ is assumed to be invariant, i.e. $d\left(x, x^{\prime}\right)=d\left(x u, x^{\prime} u\right)=d\left(u x, u x^{\prime}\right)$ for all $x, x^{\prime}, u \in X$. If $Y$ is a second compact group and $\alpha: X \rightarrow Y$ a continuous homomorphism we denote by $\operatorname{ker}(\alpha)$ the kernel of $\alpha$.

We write Aut $(X)$ for the group of continuous automorphisms of $X$ and denote by $\operatorname{id}_{X}=\mathbf{1}_{\text {Aut }(X)}$ the trivial automorphism of $X$. Let $W \subset V \subset X$ be subgroups of $X$ such that $W$ is normal in $V$, and let $\alpha \in$ Aut $(X)$ be an automorphism with $\alpha(V)=V$ and $\alpha(W)=W$. Then $\alpha_{V}$ and $\alpha_{V / W}$ denote the automorphisms induced by $\alpha$ on $V$ and $V / W$, respectively. If $\Gamma \subset$ Aut $(X)$ and the groups $W$ and $V$ are $\Gamma$-invariant (i.e. invariant under every $\gamma \in \Gamma$ ) we set $\Gamma_{v}=\left\{\gamma_{v}: \gamma \in \Gamma\right\}$ and $\Gamma_{v / w}=\left\{\gamma_{v / w}: \gamma \in \Gamma\right\}$. 
Let $X_{i}, i=1,2$, be compact groups and let $\alpha_{i} \in$ Aut $\left(X_{i}\right)$. The pairs $\left(X_{1}, \alpha_{1}\right)$ and $\left(X_{2}, \alpha_{2}\right)$ are conjugate if there exists a continuous group isomorphism $\varphi: X_{1} \rightarrow X_{2}$ such that

$$
\varphi \cdot \alpha_{1}=\alpha_{2} \cdot \varphi
$$

More generally, if $\Gamma_{i} \subset$ Aut $\left(X_{i}\right)$ are countable groups, we say that $\left(X_{1}, \Gamma_{1}\right)$ and $\left(X_{2}, \Gamma_{2}\right)$ are conjugate if there exist continuous group isomorphisms $\varphi: X_{1} \rightarrow X_{2}$ and $\theta: \Gamma_{1} \rightarrow \Gamma_{2}$ such that $\varphi \cdot \gamma=\theta(\gamma) \cdot \varphi$ for all $\gamma \in \Gamma$.

Let $G$ be a compact group, $D$ a countable set, and let $G^{D}$ be the compact group of maps $x: D \rightarrow G$. For every $E \subset D$ we denote by $\pi_{E}: G^{D} \rightarrow G^{E}$ the projection

$$
\pi_{E}(x)=\left.x\right|_{E}, \quad x \in G^{D},
$$

where $\left.x\right|_{E}$ is the restriction of the map $x: D \rightarrow G$ to $E$. A subgroup $V \subset G^{D}$ will be called full if it is closed, and if $\pi_{\{d\}}(V)=G$ for every $d \in D$.

Now assume that $\Gamma$ is a countable group and consider the shift (or Bernoulli) action of $\Gamma$ on $G^{\Gamma}$ given by

$$
\left(T_{\gamma} x\right)\left(\gamma^{\prime}\right)=x\left(\gamma^{\prime} \gamma\right)
$$

for every $x \in G^{\mathrm{r}}, \gamma, \gamma^{\prime} \in \Gamma$. Clearly

$$
T_{\mathrm{\Gamma}}=\left\{T_{\gamma}: \gamma \in \Gamma\right\} \subset \operatorname{Aut}\left(G^{\mathrm{I}}\right) \text {. }
$$

A closed subgroup $V \subset G^{\Gamma}$ is shift-invariant if $T_{\gamma} V=V$ for all $\gamma \in \Gamma$. If $V \subset G^{\Gamma}$ is a closed, shift-invariant subgroup and $\gamma \in \Gamma$, the restriction of $T_{\gamma}$ to $V$ will again be denoted by $T_{\gamma}$.

If $X$ is a compact group and $\Gamma \subset$ Aut $(X)$ a countable group, then $(X, \Gamma)$ is conjugate to a closed, shift-invariant subgroup $Y \subset G^{\Gamma}$ if there exists a continuous group isomorphism $\varphi: X \rightarrow Y$ such that

$$
\varphi \cdot \gamma=T_{\gamma} \cdot \varphi
$$

for all $\gamma \in \Gamma$. The map $\varphi$ in $(2.5)$ is a conjugacy of $(X, \Gamma)$ into $G^{l}$.

Let $X$ be a compact group and let $\Gamma \subset \operatorname{Aut}(X)$ be a countable subgroup. The Haar measure $\lambda_{X}$ of $X$ is obviously invariant under $\Gamma$. We call $\Gamma$ ergodic if it acts ergodically on the probability space $\left(X, \lambda_{X}\right)$, and topologically transitive if there exists a point $x \in X$ such that the orbit $\Gamma x=\{\gamma(x): \gamma \in \Gamma\}$ is dense in $X$. If $G$ is a compact group and $V \subset G^{r}$ a closed, shift invariant subgroup, then $V$ is ergodic or topologically transitive if the action of $T_{\mathrm{I}}$ on $V$ is ergodic or topologically transitive. The following result was proved by $\mathrm{D}$. Berend in [Be] under the more general assumption that $\Gamma$ is an arbitrary semigroup of continuous epimorphisms of $X$.

THEOREM 2.1. Let $X$ be a compact group and let $\Gamma \subset$ Aut $(X)$ be a countable group. Then $\Gamma$ is ergodic if and only if it is topologically transitive.

Theorem 2.1 is a consequence of the following lemma.

LeMMA 2.2. Let $X$ be a compact group with Haar measure $\lambda_{X}$, and let $\Gamma \subset$ Aut $(X)$ be a countable group. The following conditions are equivalent.

(1) $\Gamma$ is nonergodic on $X$; 
(2) there exists a nontrivial, continuous, irreducible, unitary representation $\sigma$ of $X$ on a (finite dimensional) complex Hilbert space $\mathscr{K}$ such that the group

$$
\Gamma_{\sigma}=\{\gamma \in \Gamma: \text { the representation } \sigma \cdot \gamma \text { is unitarily equivalent to } \sigma\}
$$

has finite index in $\Gamma$;

(3) there exists a compact Lie group $Y \neq\{1\}$, a continuous, surjective homomorphism $\eta: X \rightarrow Y$, and a homomorphism $\kappa: \Gamma \rightarrow$ Aut $(Y)$ such that

$$
\eta \cdot \gamma=\kappa(\gamma) \cdot \eta
$$

for every $\gamma \in \Gamma$, and for which the normal subgroup

$$
\Delta=\{\gamma \in \Gamma: \kappa(\gamma) \text { is an inner automorphism of } \gamma\}
$$

has finite index in $\Gamma$;

(4) there exists a closed, normal, $\Gamma$-invariant subgroup $V \subsetneq X$ and a metric on $X / V$ which is invariant under $\Gamma_{x / v}$.

Proof. Assume that $\Gamma \subset \operatorname{Aut}(X)$ is nonergodic. We put $\mathscr{H}=$ $\left\{f \in L^{2}\left(X, \lambda_{X}\right): \int f d \lambda_{X}=0\right\}$ and denote by $\rho$ the right regular representation of $X$ on $\mathscr{H}$ defined by $(\rho(x) f)\left(x^{\prime}\right)=f\left(x^{\prime} x\right)$ for $f \in \mathscr{H}, x^{\prime}, x \in X$. Choose a nonzero $\Gamma$ invariant function $f \in \mathscr{H}$ and an irreducible $\rho$-invariant subspace $\mathscr{K} \subset \mathscr{H}$ such that the projection $P_{\mathscr{K}} f$ of $f$ onto $\mathscr{K}$ is nonzero. If $\sigma=P_{\mathscr{K}} \cdot \rho$, consider the collection $\left\{\sigma \cdot \gamma^{-1}: \gamma \in \Gamma\right\}$ of irreducible, unitary representations of $X$ on $\mathscr{K}$ given by $\sigma \cdot \gamma^{-1}(x)=\sigma\left(\gamma^{-1}(x)\right), x \in X, \gamma \in \Gamma$. For every $\gamma \in \Gamma, \sigma \cdot \gamma^{-1}$ is unitarily equivalent to the restriction $\rho_{\gamma}$ of $\rho$ to $\mathscr{K}(\gamma)=\left\{f \cdot \gamma^{-1}: f \in \mathscr{K}\right\} \subset \mathscr{H}$. If $\sigma \cdot \gamma^{-1}$ is unitarily inequivalent to $\sigma, \mathscr{K}(\gamma)$ must be orthogonal to $\mathscr{K}$, but the $\Gamma$-invariance of $f$ implies that $\left\|P_{\mathscr{H}(\gamma)} f\right\|=\left\|P_{\mathscr{J}} f\right\| \neq 0$ for every $\gamma \in \Gamma$, where $\|\cdot\|$ denotes the norm in $\mathscr{H}$. Hence the group $\Gamma_{\sigma}=\left\{\gamma \in \Gamma: \sigma \cdot \gamma^{-1}\right.$ is unitarily equivalent to $\left.\sigma\right\}$ has finite index in $\Gamma$. This proves (2).

Now assume that (2) is satisfied. Since every nontrivial, continuous, irreducible, unitary representation $\sigma$ of $X$ occurs as a subrepresentation of $\rho$ on $\mathscr{H}$, we may assume that we are in the situation described at the end of the first part of this proof and use the same notation. For every $\gamma \in \Gamma_{\sigma}$ there exists a unitary operator $V_{\gamma}: \mathscr{K} \rightarrow \mathscr{K}$ such that $V_{\gamma}^{-1} \sigma(x) V_{\gamma}=\sigma \cdot \gamma^{-1}(x)$ for every $x \in X$.

We write $F=\Gamma / \Gamma_{\sigma}$ for the space of left cosets of $\Gamma_{\sigma}$, denote by $\pi: \Gamma \rightarrow F$ the quotient map, and choose a map $c: F \rightarrow \Gamma$ with $\pi \cdot c=\mathrm{id}_{F}$ and $c\left(\Gamma_{\sigma}\right)=\mathbf{1}_{\Gamma}$. The restriction of $\rho$ to $\mathscr{L}=\bigoplus_{\delta \in c(F}, \mathscr{K}(\delta)$ is denoted by $\eta$, and a typical element $f \in \mathscr{L}$ is written as $f=\left(f_{\delta} \cdot \delta^{-1}, \delta \in c(F)\right)$ with $f_{\delta} \in \mathscr{K}$ for every $\delta \in c(F)$. For every $\gamma \in \Gamma$ we define a unitary operator $U_{\gamma}$ on $\mathscr{L}$ by setting $U_{\gamma} f=\left(\left(V_{a(\gamma, \delta)} f_{\delta}\right) \cdot c\left(\pi\left(\gamma^{-1} \delta\right)\right)^{-1}\right.$, $\delta \in c(F))$ for every $f=\left(f_{\delta} \cdot \delta^{-1}, \delta \in c(F)\right) \in \mathscr{L}$, where $a(\gamma, \delta)=\gamma^{-1} \delta c\left(\pi\left(\gamma^{-1} \delta\right)\right)^{-1} \in$ $\Gamma_{\sigma}$ for every $\gamma \in \Gamma, \delta \in c(F)$. It is clear that, for every $\gamma \in \Gamma$ and $x \in X$,

$$
U_{\gamma}^{-1} \eta(x) U_{\gamma}=\eta \cdot \gamma^{-1}(x) .
$$

Put $Y=\eta(X) \subset \mathscr{U}(\mathscr{L})$, where $U(\mathscr{L})$ denotes the group of unitary operators on the finite dimensional Hilbert space $\mathscr{L}$, observe that $Y$ is a compact Lie group, and define a homomorphism $\kappa: \Gamma \rightarrow$ Aut $(Y)$ by setting $\kappa(\gamma)(y)=U_{\gamma}^{-1} y U_{\gamma}$ for every $\eta \in Y$ and $\gamma \in \Gamma$ (cf. (2.7)). We furnish $Y$ with the metric induced by the operator norm on $U(\mathscr{L})$ and note that $\kappa(\gamma)$ is an isometry of $Y$ for every $\gamma \in \Gamma$. Since the group 
of isometric automorphisms of the compact Lie group $Y$ (with respect to a fixed metric on $Y$ ) is again a compact Lie group, and since the group Inn $(Y)$ of inner automorphisms of $Y$ is an open subgroup of the group of isometric automorphisms of $Y$, we conclude that the subgroup $\Delta=\{\gamma \in \Gamma: \kappa(\gamma) \in \operatorname{Inn}(Y)\}$ has finite index in $\Gamma$. This shows that (3) is a consequence of (2).

If we can find $Y, \eta$ and $\kappa$ as in (3), there exists a metric $d$ on $Y$ which is invariant under every $\kappa(\gamma), \gamma \in \Gamma$, and we obtain a $\Gamma_{X / V}$-invariant metric on $X / V \cong Y$ where $V=\operatorname{ker}(\eta)$, as claimed in (4).

Finally, if (4) is satisfied, we write $\eta: X \rightarrow X / V$ for the quotient map, choose $\varepsilon>0$ such that $B=\left\{x \in X: d\left(1_{X / v}, \eta(x)\right) \leq \varepsilon\right\} \neq X$, and note that $B$ is $\Gamma$-invariant. Hence $\Gamma$ is not ergodic.

Proof of Theorem 2.1. If $\Gamma$ is ergodic it is topologically transitive. Conversely, if $\Gamma$ is nonergodic, Lemma 2.2 implies the existence of a closed, normal, $\Gamma$-invariant subgroup $V \subsetneq X$ such that $\Gamma_{X / V}$ acts isometrically on $X / V$ for some suitable metric on $X / V$. The automorphism group $\Gamma_{X / V}$ is thus not topologically transitive (cf the last part of the proof of Lemma 2.2), which implies that $\Gamma$ is not topologically transitive on $X$.

THEOREM 2.3. Let $X$ be a compact group with Haar measure $\lambda_{X}$, and let $\Gamma \subset$ Aut $(X)$ be a countable group. Then there exists a countable ordinal $\omega=\{\alpha: \alpha<\omega\}$ and $a$ collection $\left\{V_{\alpha}: \alpha<\omega\right\}$ of closed, $\Gamma$-invariant subgroups of $X$ with the following properties:

(1) $V_{0}=X$;

(2) if $0 \leq \alpha<\alpha+1<\omega$ then $V_{\alpha+1}$ is a proper normal subgroup of $V_{\alpha}, V_{\alpha} / V_{\alpha+1}$ is a Lie group, and there exists a metric on $V_{\alpha} / V_{\alpha+1}$ which is invariant under $\Gamma_{V_{\alpha} / V_{\alpha+1}}$;

(3) if $\alpha$ is a limit ordinal, then $V_{\alpha}=\bigcap_{0 \leq \beta<\alpha} V_{\beta}$;

(4) the action of $\Gamma$ on $X^{\prime}=\bigcap_{0 \leq \alpha<\omega} V_{\alpha}$ is ergodic, and $X^{\prime}$ is a maximal ergodic subgroup of $X$.

Proof. The subgroups $\left\{V_{\alpha}: \alpha \in \omega\right\}$ satisfying (1)-(3) are obtained from Lemma 2.2 by a (necessarily countable) transfinite induction argument. In order to prove (4) we assume that $W$ is a closed, $\Gamma$-invariant subgroup of $X$ with $W \supsetneq X^{\prime}$, and that $\Gamma_{W}$ is ergodic. Then $\lambda_{W}\left(X^{\prime}\right)=0$, and we set

$$
\alpha_{0}=\min \left\{\alpha: \alpha<\omega \text { and } \lambda_{W}\left(W \cap V_{\alpha}\right)=0\right\} \quad \text { if this is nonempty, }
$$

and

$$
\alpha_{0}=\omega \text { otherwise. }
$$

If $\alpha_{0}$ is a limit ordinal then there exists a $\beta<\alpha_{0}$ with $0<\lambda_{W}\left(W \cap V_{\beta}\right)<1$, contrary to the ergodicity of $\Gamma$ on $W$. Hence $\alpha_{0}$ is not a limit ordinal, and the ergodicity of $\Gamma$ implies that $W \cap V_{\alpha_{0}-1}$ is an open, $\Gamma$-invariant subgroup of $W$ and hence equal to $W \subset V_{\alpha_{0}-1}$. From the definition of $\alpha_{0}$ we know that $W$ is not contained in $V_{\alpha_{0}}$, and (2) implies that $W$ has nontrivial open, $\Gamma$-invariant subsets, which is absurd. Hence $\Gamma_{W}$ cannot be ergodic, as claimed in [4].

If $\Gamma$ is a countable group and $(X, \mathscr{S}, \mu)$ a probability space, a measure preserving action $(\gamma, x) \rightarrow R_{\gamma} x$ of $\Gamma$ on $(X, \mathscr{S}, \mu)$ is (strongly) mixing if $\lim _{\gamma \rightarrow \infty} \mu\left(B \cap R_{\gamma} C\right)=$ $\mu(B) \mu(C)$ for all $B, C \in \mathscr{S}$ (cf. e.g. [Dy, S1]). 
THEOREM 2.4. Let $X$ be a compact group with Haar measure $\lambda_{X}$ and let $\Gamma \subset$ Aut $(X)$ be a finitely generated, infinite, abelian group. If $\gamma \in \Gamma$ is an element of infinite order then $\gamma$ is mixing if and only if it is ergodic, and the action of $\Gamma$ on $\left(X, \lambda_{X}\right)$ is mixing if and only if every element $\gamma \in \Gamma$ of infinite order is ergodic (or mixing).

Proof. If $\sigma$ is a nontrivial, continuous, irreducible, unitary representation of $X$ we define $\Gamma_{\sigma}$ by (2.6). From the proof of Lemma 2.2 we see that $\Gamma$ is mixing if and only if $\Gamma_{\sigma}$ is a finite subgroup of $\Gamma$ for every such $\sigma$. The assertion of the theorem is an obvious consequence of this observation.

Remark 2.5. The connection between ergodicity, mixing, and orbits of irreducible representations for automorphisms of compact groups was first pointed out in [Ha] (in the abelian case) and [Ka] (in the nonabelian case).

\section{The descending chain condition}

We leave the discussion of general groups of automorphisms of a compact group $X$ and impose a further condition on the pair $(X, \Gamma)$ which turns out to have some interesting consequences.

Definition 3.1. Let $X$ be a compact group and let $\Gamma \subset$ Aut $(X)$ be a countable group. The pair $(X, \Gamma)$ satisfies the descending chain condition (or, equivalently, $X$ satisfies the descending chain condition for $\Gamma$-invariant subgroups) if there exists, for every sequence $X \supset V(1) \supset V(2) \supset \cdots \supset V(n) \supset \cdots$ of closed, $\Gamma$-invariant subgroups, an integer $N \geq 1$ with $V(n)=V(N)$ for all $n \geq N$.

The main aim of this section is to prove the following result.

THEOREM 3.2. Let $X$ be a compact group and let $\Gamma \subset$ Aut $(X)$ be a finitely generated, abelian group. Then $(X, \Gamma)$ satisfies the descending chain condition if and only if there exists a compact Lie group $G$ such that $(X, \Gamma)$ is conjugate to a shift-invariant subgroup $Y \subset G^{r}$.

For the proof of Theorem 3.2 we require four lemmas, the first of which is obvious.

LeMmA 3.3. Let $X$ be a compact group, $\Gamma \subset \operatorname{Aut}(X)$ a countable group, and let $D$ be a countable set. We define $\Gamma^{D}=\left\{\gamma^{D}: \gamma \in \Gamma\right\} \subset$ Aut $\left(X^{D}\right)$ by setting, for every $\gamma \in \Gamma$, $y \in X^{D}$,

$$
\left(\gamma^{D}(y)\right)(i)=\gamma(y(i)), i \in D .
$$

If $D$ is finite and $(X, \Gamma)$ satisfies the descending chain condition, then $\left(X^{D}, \Gamma^{D}\right)$ again satisfies the descending chain condition. In other words, the descending chain condition is inherited by finite cartesian products.

For the next two lemmas assume that $X$ is a compact group and $\Gamma \subset \operatorname{Aut}(X)$ a countable group such that $(X, \Gamma)$ satisfies the descending chain condition. Put $Y=X^{\mathbb{Z}}$ and write $\Pi_{k}=\pi_{\{0, \ldots, k\}}: Y \rightarrow X^{k+1}, k \geq 1$, for the projection onto the coordinates $\{0, \ldots, k\}$ (cf. (2.2)). We define $\Gamma^{\mathbb{Z}}=\left\{\gamma^{\mathbb{Z}}: \gamma \in \Gamma\right\}$ by (3.1) and denote by $\left\{T_{n}: n \in\right.$ $\mathbb{Z}\}$ the shifts on $Y$ given as in (2.3) by $\left(T_{n} y\right)(m)=y(n+m), m, n \in \mathbb{Z}, y \in Y$. Clearly, $T_{n} \cdot \gamma^{\mathbb{Z}}=\gamma^{\mathbb{Z}} \cdot T_{n}$ for every $n \in \mathbb{Z}, \gamma \in \Gamma$, and we set

$$
\Delta=\left\{T_{n} \cdot \gamma^{\mathbb{Z}}: n \in \mathbb{Z}, \gamma \in \Gamma\right\} \subset \operatorname{Aut}(Y) .
$$


LEMMA 3.4. Let $V$ be a $\Delta$-invariant subgroup of $Y=X^{\mathbb{Z}}$. For every $k \geq 1$ consider the closed, $\Gamma$-invariant subgroup

$$
X(k)=\left\{y(k): y \in V \quad \text { and } \quad y(0)=y(1)=\cdots=y(k-1)=1_{X}\right\} \subset X
$$

(note that $X(k)$ is the usual follower set of $\left[1_{X}, \ldots, 1_{X}\right]$ ). Then $X(k) \supset X(k+1)$ for all $k \geq 1$, and there exists $a K \geq 1$ with $X(k)=X(K)$ for all $k \geq K$. Put

$$
H=\Pi_{K}(V) \subset X^{K+1} \text {. }
$$

Then

$$
V=\left\{x \in Y: \Pi_{K}\left(T_{m} x\right) \in H \quad \text { for all } m \in \mathbb{Z}\right\} .
$$

Proof. Since $(X, \Gamma)$ satisfies the descending chain condition, there exists a $K \geq 1$ with $X(k)=X(K)=X^{\prime}$, say, for all $k \geq K$. We set $H=\Pi_{K}(V)$ and observe that

$$
H^{\prime}=\Pi_{K}\left(\left\{y \in V: \Pi_{K-1}(y)=\left(1_{X}, \ldots, 1_{X}\right)\right\}\right)=\left\{\left(1_{X}, \ldots, 1_{X}, x\right): x \in X^{\prime}\right\} \subset X^{K+1}
$$

is a closed, normal subgroup of $H$, and that

$$
x_{K} \in x_{K}^{\prime} \cdot X^{\prime}
$$

whenever $\left(x_{0}, x_{1}, \ldots, x_{K}\right),\left(x_{0}^{\prime}, x_{1}^{\prime}, \ldots, x_{K}^{\prime}\right) \in H$ satisfy that $x_{i}=x_{i}^{\prime}$ for $0 \leq i<K$ (in other words, the follower set of $\left[x_{0}, \ldots, x_{K-1}\right]$ is a coset of the follower set $X^{\prime}$ of $\left.\left[\mathbf{1}_{X}, \ldots, \mathbf{1}_{X}\right]\right)$. Our choice of $X^{\prime}$ also implies that there exists, for every $x \in X^{\prime}, y \in V$, and $n \in \mathbb{Z}$, an element $z \in V$ with $z(m)=y(m)$ for $m<n$ and

$$
z(n)=y(n) \cdot x \text {. }
$$

Put

$$
Y_{H}=\left\{x \in Y: \Pi_{K}\left(T_{m} x\right) \in H \text { for all } m \in \mathbb{Z}\right\} .
$$

In order to verify that $V=Y_{H}$ we first note that $Y_{H}$ is shift-invariant, and that $V \subset Y_{H}$. Conversely, if $z \in Y_{H}$, there exists an element $y_{0} \in Y$ such that $\Pi_{K}(z)=$ $\Pi_{K}\left(y_{0}\right)$. From (3.3) and (3.4) we know that we can find $y_{1} \in Y$ with $y_{1}(m)=y_{0}(m)$ for all $m \leq K$ and $y_{1}(K+1)=z(K+1)$. By repeating this argument we obtain a sequence $\left(y_{1}, 1 \geq 0\right)$ in $Y$ such that $y_{1}(m)=z(m)$ for all $1 \geq 0$ and $m \leq K+1$. Since $V$ is closed there exists a $y \in V$ with $y(m)=z(m)$ for all $m \geq 0$.

We have proved that the set $P(z)=\{y \in Y: y(m)=z(m)$ for all $m \geq 0\}$ is a closed, nonempty subset for every $z \in Y_{H}$. For every $z \in Y_{H}$, the intersection $Q(z)=$ $\bigcap_{n \geq 0} T_{n}\left(P\left(T_{-n} z\right)\right) \subset Y$ is again nonempty, and $Q(z)=\{z\}$. This shows that $Y_{H} \subset V$, i.e. that $V=Y_{H}$.

Lемма 3.5. The group $Y=X^{\mathbb{Z}}$ satisfies the descending chain condition for $\Delta$-invariant subgroups.

Proof. We use the notation of Lemma 3.4. Let $(V(n), n \geq 1)$ be a decreasing sequence of closed, $\Delta$-invariant subgroups of $Y$. We fix $n$ for the moment and define a sequence of closed, $\Gamma$-invariant subgroups $(X(n, k), k \geq 1)$ of $X$ by (3.2) with $V(n)$ replacing $V$. Our assumptions imply that, for all $k, n \geq 1, X(n, k) \supset X(n+1, k)$ and $X(n, k) \supset$ $X(n, k+1)$. Since $(X, \Gamma)$ satisfies the descending chain condition there exists, for every $n \geq 1$, an integer $K(n) \geq 1$ with $X_{n}:=X(n, K(n))=X(n, k)$ for all $k \geq K(n)$. 
Since $X_{n} \supset X_{n+1}$ for all $n \geq 1$, we may apply the same argument once again to obtain an integer $N \geq 1$ with $X^{\prime}:=X_{N}=X_{n}$ for all $n \geq N$. Put $K=\max _{1 \leq n \leq N} K(n)$ and note that $X(n, k)=X^{\prime}$ for all $n \geq N$ and $k \geq K$. Let $D=\{0, \ldots, K\}$. For every $n \geq 1$, the group $H(n)=\Pi_{K}(V(n)) \subset X^{K+1}=X^{D}$ is $\Gamma^{D}$-invariant, and the sequence $(H(n), n \geq 1)$ decreases. By Lemma 3.3 there exists an $N^{\prime} \geq N$ with $H(n)=H\left(N^{\prime}\right)=$ $H$ for all $n \geq N^{\prime}$. From Lemma 3.4 we know that $V(n)=Y_{H(n)}$ for all $n \geq N$ (cf. (3.5)) and conclude that $V(n)=V\left(N^{\prime}\right)$ for all $n \geq N^{\prime}$. This shows that the sequence $(V(n), n \geq 1)$ is eventually constant and hence that $(Y, \Delta)$ satisfies the descending chain condition.

LEMMA 3.6. If $G$ is a compact Lie group and $d \geq 1$ then $G^{\mathbb{Z}^{4}}$ satisfies the descending chain condition for shift-invariant subgroups.

Proof. This lemma is proved by induction. Since $G$ is a Lie group, every decreasing sequence of closed subgroups of $G$ eventually becomes constant, so that we may apply Lemma 3.4 with $X=G$ and $\Gamma=\left\{\mathrm{id}_{G}\right\}$ to conclude that $G^{\mathbb{Z}}$ satisfies the chain condition for shift-invariant subgroups. Now assume that $m \geq 1$, and that the group $G^{\mathbb{Z}^{\prime \prime}}$ satisfies the descending chain condition for shift-invariant subgroups for every compact Lie group $G$. Since $G^{\mathbb{Z}^{m+1}}=\left(G^{\mathbb{Z}^{m}}\right)^{\mathbb{Z}}$, Lemma 3.4 (with $X=G^{\mathbb{Z}^{m}}$ and $\Gamma=T_{\mathbb{Z}^{m}}$ ) implies that $G^{\mathbb{Z}^{m+1}}$ satisfies the descending chain condition for shift-invariant subgroups. This proves the assertion of the lemma.

Proof of Theorem 3.2. First assume that $(X, \Gamma)$ satisfies the descending chain condition. Since $X$ is compact there exists a sequence of (finite dimensional) continuous, irreducible, unitary representations $\left(\rho_{n}, n \geq 1\right)$ of $X$ which together separate the points of $X$. For every $n \geq 1$ we put $\sigma_{n}=\rho_{1} \oplus \rho_{2} \oplus \cdots \oplus \rho_{n}$, denote by $G_{n}$ the compact Lie group $\sigma_{n}(X)=\left\{\sigma_{n}(x): x \in X\right\}$, and define a continuous group homomorphism $\varphi_{n}: X \rightarrow G_{n}^{\Gamma}$ by setting, for every $x \in X, \gamma \in \Gamma, \varphi_{n}(x)(\gamma)=\sigma_{n}(\gamma(x))$. Then $\varphi_{n}(\gamma(x))=$ $T_{\gamma} \varphi_{n}(x)$ for every $x \in X, \gamma \in \Gamma$, and $\operatorname{ker}\left(\varphi_{n}\right)$ is a closed, $\Gamma$-invariant, normal subgroup of $X$. Since $\operatorname{ker}\left(\varphi_{1}\right) \supset \operatorname{ker}\left(\varphi_{2}\right) \supset \cdots \supset \operatorname{ker}\left(\varphi_{n}\right) \supset \cdots$ for every $n \geq 1$ and $\bigcap_{n \geq 1} \operatorname{ker}\left(\varphi_{n}\right)=\left\{\mathbf{1}_{X}\right\}$, the descending chain condition implies that $\operatorname{ker}\left(\varphi_{m}\right)=\left\{\boldsymbol{1}_{X}\right\}$ for some $m \geq 1$, i.e. that $\varphi_{m}$ is a topological isomorphism of $X$ onto a closed, shift-invariant subgroup $V \subset G_{m}^{\Gamma}$, where $G_{m}$ is a Lie group.

The proof is completed by showing that, for every Lie group $G$, and for every finitely generated, abelian group $\Gamma$, the group $Y=G^{\Gamma}$ satisfies the descending chain condition for shift-invariant subgroups. We choose an integer $d \geq 1$ and a surjective homomorphism $\zeta: \mathbb{Z}^{d} \rightarrow \Gamma$ and define a continuous, injective homomorphism $\xi: G^{\Gamma} \rightarrow$ $G^{\mathbb{Z}^{d}}$ by setting, for every $x \in G^{\Gamma}$ and every $\gamma \in \mathbb{Z}^{d}, \xi(x)(\gamma)=x(\zeta(\gamma))$. For every closed, shift-invariant subgroup $Y \subset G^{\Gamma^{\top}}$, the closed subgroup $\xi(Y) \subset G^{\mathbb{Z}^{d}}$ is again shift-invariant, and the descending chain condition for shift-invariant subgroups of $G^{Z^{d}}$ implies that $G^{\Gamma}$ also satisfies the descending chain condition.

For the remainder of this section we assume that $\Gamma$ is an infinite, finitely generated, abelian group. 
Corollary 3.7. Let $X$ be a compact group, and $\Gamma \subset$ Aut $(X)$. The following conditions are equivalent.

(1) $(X, \Gamma)$ satisfies the descending chain condition;

(2) the group $X$ satisfies the descending chain condition for closed, normal, $\Gamma$-invariant subgroups;

(3) there exists a compact Lie group $G$ such that $(X, \Gamma)$ is conjugate to a full, shift-invariant subgroup of $G^{\mathrm{r}}$.

Proof. The implications (3) $\Rightarrow(1) \Rightarrow(2)$ are either obvious or part of Theorem 3.2. The final implication $(2) \Rightarrow(3)$ is a consequence of the first part of the proof of Theorem 3.2.

Corollary 3.8. Let $G$ be a compact Lie group. If $n \geq 1$ and $Y_{1}, \ldots, Y_{n}$ are closed, shift-invariant subgroups of $G^{\Gamma}$ there exists a finite set $D \subset \Gamma$ with the following property: for every $k=1, \ldots, n$,

$$
Y_{k}=\left\{x \in G^{\Gamma}: \pi_{D}\left(T_{\gamma} x\right) \in H_{k} \quad \text { for every } \gamma \in \Gamma\right\},
$$

where

$$
H_{k}=\pi_{D}\left(Y_{k}\right)
$$

Proof. Let $(D(m), m \geq 1)$ be an increasing sequence of finite subsets of $\Gamma$ with $\bigcup_{m} D(m)=\Gamma$. For every $m \geqslant 1$ and $k=1, \ldots, n$, we have that

$$
Y_{k, m}=\left\{x \in G^{\Gamma}: \pi_{D(m)}\left(T_{\gamma} x\right) \in \pi_{D(m)}\left(Y_{k}\right) \text { for every } \gamma \in \Gamma\right\} \supset Y_{k},
$$

and $\bigcap_{m} Y_{k, m}=Y_{k}$. The descending chain condition implies that there exists an $m^{\prime} \geq 1$ with $Y_{k, m^{\prime}}=Y_{k}$ for $k=1, \ldots, n$, and $D=D\left(m^{\prime}\right)$ satisfies (3.6) and (3.7).

COROLlary 3.9. Let $G$ be a compact Lie group. If $Y \subset G^{\Gamma}$ is a closed shift-invariant subgroup there exists a finite set $D \subset \Gamma$ with the following properties.

$$
Y=\left\{x \in G^{\Gamma}: \pi_{D}\left(T_{\gamma} x\right) \in H \text { for every } \gamma \in \Gamma\right\}
$$

where $H=\pi_{D}(Y)$ is a closed subgroup of $G^{D}$;

(2) if $Y^{0}$ and $H^{0}$ denote the connected components of the identity in $Y$ and $H$, respectively, then $\pi_{D}\left(Y^{0}\right)=H^{0}$, and

$$
\begin{gathered}
Y^{0}=\left\{x \in G^{\Gamma}: \pi_{D}\left(T_{\gamma} x\right) \in H^{0} \quad \text { for every } y \in \Gamma\right\} ; \\
C(Y)=\left\{x \in G^{\mathrm{r}}: \pi_{D}\left(T_{\gamma} x\right) \in C(H) \quad \text { for every } \gamma \in \Gamma\right\} .
\end{gathered}
$$

Proof. Corollary 3.8 implies that there exists a finite set $D \subset \Gamma$ such that

$$
\begin{aligned}
Y & =\left\{x \in G^{\mathrm{l}}: \pi_{D}\left(T_{\gamma} x\right) \in \pi_{D}(Y) \quad \text { for every } \gamma \in \Gamma\right\}, \\
Y^{0} & =\left\{x \in G^{\mathrm{l}}: \pi_{D}\left(T_{\gamma} x\right) \in \pi_{D}\left(Y^{0}\right) \quad \text { for every } \gamma \in \Gamma\right\},
\end{aligned}
$$

and

$$
C(Y)=\left\{x \in G^{\mathrm{Y}}: \pi_{D}\left(T_{\gamma} x\right) \in \pi_{D}(C(Y)) \quad \text { for every } \gamma \in \Gamma\right\} .
$$

Clearly $\pi_{D}(C(Y)) \subset C\left(\pi_{D}(Y)\right), C(Y)=\left\{x \in G^{\mathrm{I}}: \pi_{D}\left(T_{\gamma} x\right) \in C\left(\pi_{D}(Y)\right)\right.$ for every $\gamma \in \Gamma\}, \pi_{D}\left(Y^{0}\right) \subset\left(\pi_{D}(Y)\right)^{0}$ (the connected component of the identity in $\pi_{D}(Y)$ ), and $\pi_{D}\left(Y^{0}\right)$ is an open subgroup of $\pi_{D}(Y)$. Hence $\pi_{D}\left(Y^{0}\right)=\left(\pi_{D}(Y)\right)^{0}$.

Remarks 3.10. (1) If $D^{\prime} \subset \Gamma$ is a finite set with $D \subset D^{\prime}$ then $D^{\prime}$ satisfies (3.6)-(3.10). 
(2) Let $G$ be a compact Lie group. The corollaries 3.8-3.9 show that the closed, shift-invariant subgroups of $G^{\Gamma}$ are in a natural sense subshifts of finite type (or Markov subshifts) of $G^{r}$.

Corollary 3.11. Let $K$ be a compact Lie group and let $Y_{1}, \ldots, Y_{n}$ be closed, shift-invariant subgroups of $K^{\mathbb{Z}^{d}}$. Then there exists a compact Lie group $G$ and an isomorphism $\varphi$ of $K^{\mathbb{Z}^{d}}$ into $G^{\mathbb{Z}^{d}}$ such that, for $k=1, \ldots, n$,

$$
\varphi\left(Y_{k}\right)=\left\{x \in G^{\mathbb{Z}^{d}}: \pi_{I(d)}\left(T_{n} x\right) \in H_{k} \quad \text { for every } n \in \mathbb{Z}^{d}\right\}
$$

where

$$
I(d)=\{0,1\}^{d}
$$

and

$$
H_{k}=\pi_{I(d)}\left(\varphi\left(Y_{k}\right)\right) \subset G^{I(d)} .
$$

Proof. Choose $D$ and $H_{k}, 1 \leq k \leq n$, as in Corollary 3.8 and set $G=K^{d}$. We define a continuous, injective homomorphism $\varphi: Y \rightarrow G^{\mathbb{Z}^{\prime \prime}}$ by setting $\varphi(x)(n)=\pi_{D}\left(T_{n} x\right)$ for every $x \in K^{\mathbb{Z}}, n \in \mathbb{Z}^{d}$. According to remark 3.10(1) the groups $\varphi\left(Y_{k}\right)$ satisfy (3.11)-(3.13).

Proposition 3.12. Let $X$ be a zero dimensional compact group. $\Gamma \subset$ Aut $(X)$, and assume that $(X, \Gamma)$ satisfies the descending chain condition. If $G$ is a compact Lie group such that $(X, \Gamma)$ is conjugate to a full, shift-invariant subgroup $Y$ of $G^{\Gamma}$ then $G$ is finite.

Proof. Since $G$ is a Lie group and a homomorphic image of the zero dimensional group $X, G$ must be finite.

For abelian groups $X$ and $\Gamma=\mathbb{Z}$, the descending chain condition on $(X, \Gamma)$ is equivalent to a condition which appears in the literature (cf [Ln] and $[\mathbf{L P}]$ ).

Proposition 3.13. If $X$ is a compact group and $\Gamma \subset$ Aut $(X)$, then $(X, \Gamma)$ satisfies the descending chain condition if and only if there exist finitely many continuous, irreducible, unitary representations $\tau_{1}, \ldots, \tau_{n}$ of $X$ such that the representations $\left\{\tau_{i} \cdot \gamma: \gamma \in \Gamma, 1 \leq i \leq n\right\}$ together separate the points of $X$. In particular, if $X$ is abelian, $(X, \Gamma)$ satisfies the descending chain condition if and only if the dual group $X^{\wedge}$ of $X$ is finitely generated under $\Gamma$ (i.e. there exist characters $\chi_{1}, \ldots, \chi_{n} \in X^{\wedge}$ such that $\left\{\chi_{i} \cdot \gamma: 1 \leq i \leq n, \gamma \in \Gamma\right\}$ generates $\left.X^{\wedge}\right)$.

Proof. If $(X, \Gamma)$ satisfies the descending chain condition we may regard $X$ as a full, shift-invariant subgroup of $G^{\mathrm{I}}$, where $G$ is a compact Lie group (cf Theorem 3.2). Since $G$ possesses finitely many irreducible representations $\sigma_{1}, \ldots, \sigma_{n}$ which together separate points, the representations $\tau_{i}=\sigma_{i} \cdot \pi_{\{0\}}, 1 \leq i \leq n$, of $X$ have the property that $\left\{\tau_{i} \cdot \gamma: \gamma \in \Gamma, 1 \leq i \leq n\right\}$ separates the points of $X$.

Conversely, if $\tau_{1}, \ldots, \tau_{n}$ are continuous, irreducible, unitary representations of $X$ with that property, put $\tau=\tau_{1} \oplus \tau_{2} \oplus \cdots \oplus \tau_{n}$. Then $G=\tau(X)$ is a compact Lie group, and the homomorphism $\tau: X \rightarrow G^{\mathrm{I}}$ given by $\tau(x)(\gamma)=\tau(\gamma(x)), \gamma \in \Gamma, x \in X$, separates points and embeds $X$ as a full, shift-invariant subgroup of $G^{\Gamma}$. By Theorem $3.2,(X, \Gamma)$ satisfies the descending chain condition. 
The statement about abelian groups is verified by noting that, if $X$ is abelian, the characters $\left\{\chi_{i} \cdot \gamma: 1 \leq i \leq n, \gamma \in \Gamma\right\}$ generate $X^{\wedge}$ if and only if they separate the points of $X$.

LEMMA 3.14. Let $X$ be a compact group, $\Gamma \subset$ Aut $(X)$ a countable group, $V \subset X$ a closed, normal, $\Gamma$-invariant subgroup, and let $\mathrm{W} \subset \mathrm{V}$ be a $\Gamma$-invariant subgroup which is open in $V$. Then there exists a $\Gamma$-invariant subgroup $Y \subset W \subset V \subset X$ which is normal in $X$ and open in $V$.

Proof. We choose a (translation invariant) metric $d$ on $X$ and an $\varepsilon>0$ such that $B(\varepsilon)=\left\{x \in V: d\left(1_{X}, x\right)<\varepsilon\right\} \subset W$ and observe that $Y=\bigcap_{x \in X} x W x^{-1}$ is a $\Gamma$ invariant, normal subgroup of $X$, and that $B(\varepsilon) \subset Y \subset W \subset V$.

By specializing Theorem 2.3 we obtain the following result.

TheOREM 3.15. Let $X$ be a compact group, $\Gamma \subset$ Aut $(X)$, and assume that $(X, \Gamma)$ satisfies the descending chain condition. Then there exist closed, $\Gamma$-invariant subgroups $X^{\prime}=V_{n} \subset V_{n-1} \subset \cdots \subset V_{0}=X$ with the following properties.

(1) $\Gamma$ is ergodic (or, equivalently, topologically transitive) on $X^{\prime}$;

(2) for $k=0, \ldots, n-1, V_{k+1}$ is a normal subgroup of $V_{k}, V_{k} / V_{k+1}$ is a Lie group, and the action of $\Gamma$ on $V_{k} / V_{k+1}$ admits an invariant metric;

(3) if $X$ is zero dimensional, then $X^{\prime}$ is an open, normal subgroup of $X$.

Proof. The descending chain condition implies that the ordinal $\omega$ in Theorem 2.3 is finite, which proves (1) and (2). If $X$ is zero dimensional, $X^{\prime}$ has finite index in $X$ by (2), and Lemma 3.14 shows that $X^{\prime}$ has a $\Gamma$-invariant subgroup $Y$ of finite index which is normal in $X$. The ergodicity of $\Gamma$ on $X^{\prime}$ implies that $X^{\prime}=Y$.

Our last theorem in this section shows that every pair $(X, \Gamma)$ is a projective limit of pairs $\left(X_{n}, \Gamma_{n}\right)$ satisfying the descending chain condition.

THEOREM 3.16. Let $X$ be a compact group and $\Gamma \subset$ Aut $(X)$. Then there exists a sequence $X \supset V_{1} \supset V_{2} \supset \cdots$ of closed, normal, $\Gamma$-invariant subgroups of $X$ with the following properties:

(1) $\bigcap_{n} V_{n}=\left\{\mathbf{1}_{X}\right\}$;

(2) for every $n \geq 1$, put $X(n)=X / V_{n}$. Then $\left(X(n), \Gamma_{X(n)}\right)$ satisfies the descending chain condition.

Proof. As in the proof of Theorem 3.2 we choose a sequence of continuous, irreducible, unitary representations $\left(\rho_{n}, n \geq 1\right)$ of $X$ which together separate the points of $X$. For every $n \geq 1$ put $\sigma_{n}=\rho_{1} \oplus \rho_{2} \oplus \cdots \oplus \rho_{n}, G_{n}=\sigma_{n}(X)$, and define $\varphi_{n}: X \rightarrow G_{n}^{1}$ by $\varphi_{n}(x)(\gamma)=\sigma_{n}(\gamma(x)), \gamma \in \Gamma, x \in X$. By Theorem 3.2, the groups $V_{n}=$ $\operatorname{ker}\left(\varphi_{n}\right), n \geq 1$, have the required properties.

\section{An approximation theorem}

Lemma 4.1. Let $H$ be a compact Lie group with centre $C(H)$, and let $A \subset C(X)$ be a connected subgroup. For every character $\chi \in A^{\wedge}$ there exists a continuous homomorphism $\zeta_{x}: H \rightarrow S^{1}=\{z \in \mathbb{C}:|z|=1\}$ and an integer $k \geq 1$ such that $\zeta_{x}(a)=\chi(a)^{k}$ for every $a \in A$.

Proof. Let $\varphi: H \rightarrow H / A$ be the quotient map, choose a Borel map $\sigma: H / A \rightarrow H$ with 
$\varphi \cdot \sigma(x)=x$ for every $x \in H / A$, and put $c(h, x)=\sigma(h x)^{-1} h \sigma(x)$ for every $h \in H$, $x \in H / A$. We denote by $U$ the unitary representation of $H$ on the Hilbert space $\mathscr{H}=L^{2}\left(H / A, \lambda_{H / A}\right)$ given by

$$
\left(U_{h} f\right)(x)=\chi\left(c\left(h, h^{-1} x\right)\right) f\left(h^{-1} x\right), \quad h \in H, \quad f \in \mathscr{H}, \quad x \in H / A .
$$

( $U$ is the representation of $H$ induced by $\chi$.) Since $A$ is central, $U_{a}=\chi(a) \cdot I$ for every $a \in A$, where $I$ is the identity operator on $\mathscr{H}$. We choose an irreducible subrepresentation $V$ of $U$ on some finite dimensional subspace $\mathscr{K} \subset \mathscr{H}$ and put $\zeta_{X}(h)=\operatorname{det}\left(V_{h}\right)$ for every $h \in H$. Then $\zeta_{\chi}: H \rightarrow S^{1}$ is a continuous homomorphism, and $\zeta_{\chi}(a)=\chi(a)^{k}$ for every $a \in A$, where $k$ is the dimension of $\mathscr{K}$.

Lemma 4.2. Let $A=\mathbb{J}^{n}, n \geq 2, F \subset$ Aut $(A)$ a finite group, and let $\{0\} \neq B \subsetneq A$ be $a$ closed, connected, F-invariant subgroup. Then there exists a closed, connected, $F$ invariant subgroup $C$ of $A$ such that $B+C=A$ and $B \cap C$ is finite.

Proof. For every $\alpha \in F$ we consider the dual automorphism $\alpha \hat{\alpha} \in \operatorname{Aut}(\hat{A})=$ $G L(n, \mathbb{Z}) \subset G L(n, \mathbb{R})$, and we set $\langle\langle\boldsymbol{u}, \boldsymbol{v}\rangle\rangle=\sum_{\alpha \in F}\left\langle\alpha^{\hat{\gamma}}(\boldsymbol{u}), \alpha^{\hat{\alpha}}(\boldsymbol{v})\right\rangle, \boldsymbol{u}, \boldsymbol{v} \in \mathbb{R}^{n}$, where $\langle\cdot, \cdot\rangle$ denotes the Euclidean inner product on $\mathbb{R}^{n}$. The annihilator subgroup $B^{\perp} \subset \mathbb{Z}^{n} \subset \mathbb{R}^{n}$ of $B$ spans a subspace $V \subset \mathbb{R}^{n}$ of dimension $d$ with $1 \leq d<n$. The subspace $W=$ $\left\{v \in \mathbb{R}^{n}:\langle\langle u, v\rangle\rangle=0\right.$ for all $\left.u \in V\right\}$ is obviously invariant under $F^{\wedge}=\left\{\alpha^{\wedge}: \alpha \in F\right\}$, and we can find a finite subset $S \subset W \cap \mathbb{Q}^{n}$ such that $S$ spans $W$ and $\alpha \hat{\alpha}(v) \in S$ for every $\alpha \in F$ and $v \in S$. Choose $k \geq 1$ such that $k v \in \mathbb{Z}^{n}$ for every $v \in S$ and denote by $\Xi$ the subgroup of $\mathbb{Z}^{n}$ generated by $\{k v: v \in S\}$. The group $B^{\perp}+\Xi$ has finite index in $\mathbb{Z}^{n}, B^{\perp} \cap \Xi=\{0\}$, and the connected component $C$ of $\Xi^{\perp}$ in $A$ is an $F$-invariant, closed, connected subgroup of $A$ such that $B \cap C$ is finite and $B+C=A$.

Lemma 4.3. Let $H$ be a compact Lie group, and let $A \subset H$ be a closed, normal, abelian subgroup. Then there exists a closed, normal subgroup $H^{\prime} \subset H$ such that $H^{\prime} \cap A$ is finite and $H^{\prime} \cdot A=H$. In particular, $H / H^{\prime}$ is abelian.

Proof. If $A^{0}$ is the connected component of the identity in $A$ then it is obviously sufficient to prove the statement of the lemma with $A^{0}$ replacing $A$, and we assume for simplicity that $A$ itself is connected. Let $G \subset H$ be the centralizer of $A$, i.e. $G=\{h \in H: h a=a h$ for every $a \in A\}$. Since the automorphism group of $A$ is discrete and $H$ is compact, the quotient group $K=H / G$ is finite with cardinality $n$, say. The group $A$ is isomorphic to $\mathbb{T}^{d}$ for some $d \geq 1$, and we choose and fix characters $\chi_{1}, \ldots, \chi_{d} \in A^{\wedge}$ such that the map $a \rightarrow\left(\chi_{1}(a), \ldots, \chi_{d}(a)\right)$ from $A$ to $\left(S^{1}\right)^{d}$ is bijective. Lemma 4.1 allows us to find an integer $k \geq 1$ and continuous homomorphisms $\zeta_{i}=\zeta_{X_{i}}: G \rightarrow S^{1}$ such that $\zeta_{i}(a)=\chi_{i}(a)^{k}$ for every $a \in A$ and $i=1, \ldots, d$. Denote by $\varphi: H \rightarrow K$ the quotient map, choose a map $\sigma: K \rightarrow H$ with $\varphi \cdot \sigma(k)=k$ for every $k \in K$, and set $c(h, k)=\sigma(h k)^{-1} h \sigma(k), h \in H, k \in K$.

Let $V$ be the unitary representation of $H$ induced by the representation $\zeta=\zeta_{1} \oplus \cdots \oplus \zeta_{d}$ of $G$ on $\mathbb{C}^{d}$. Then $V$ acts on the Hilbert space $\mathscr{H}=l^{2}(K)^{d}$ and can be written as $V=V^{(1)} \oplus \cdots \oplus V^{(d)}$, where

$$
\left(V_{h}^{(i)} f\right)(k)=\zeta_{i}\left(c\left(h, h^{-1} k\right)\right) f\left(h^{-1} k\right) \quad \text { for every } h \in H, f \in l^{2}(K), k \in K .
$$

For every $k \in K$ we write $e_{k}$ for the unit vector in $l^{2}(K)$ given by $e_{k}\left(k^{\prime}\right)=1$ if $k=k^{\prime}$, and $e_{k}\left(k^{\prime}\right)=0$ otherwise. Then $\left\{e_{k}: k \in K\right\}$ is an orthonormal basis of $l^{2}(K)$, 
and we identify each $V_{h}^{(i)}, h \in H, 1 \leq i \leq d$, with its representation as an $n \times n$-matrix in this basis. This allows us to regard $V^{(i)}, 1 \leq i \leq d$, as continuous homomorphisms from $H$ into the group $U(n)$ of unitary $n \times n$-matrices, and to consider the representation $V$ as a continuous homomorphism $V=V^{(i)} \oplus \cdots \oplus V^{(d)}: H \rightarrow U(n)^{d} \subset U(n d)$.

The restriction of $V$ to $A$ has finite kernel, and $V_{a} \in D(n d)$ for every $a \in A$, where $D(n d)$ is the group of diagonal matrices in $U(n d)$. Eq. (4.1) shows that, for every $h \in H$, there exist unique matrices $D_{h}^{(i)}, 1 \leq i \leq d$, and $P_{h}$ in $U(n)$ such that $P_{h}$ is a permutation matrix, $D_{h}^{(i)}$ is diagonal, and

$$
V_{h}^{(i)}=D_{h}^{(i)} \cdot P_{h} \text { for every } i=1, \ldots, d \text {. }
$$

We set $D_{h}=D_{h}^{(1)} \oplus \cdots \oplus D_{h}^{(d)} \in D(n d), Q_{h}=P_{h} \oplus \cdots \oplus P_{h}$, and observe that

$$
V_{h}=D_{h} \cdot Q_{h} \text {, }
$$

and that the map $h \rightarrow Q_{h}$ is a (continuous) homomorphism from $H$ into the group of permutation matrices in $U(n d)$. For every $h \in H$ we denote by $\alpha_{h}$ the automorphism of $D(n d)$ given by $\alpha_{h}(D)=V_{h} D V_{h}^{-1}=Q_{h} D Q_{h}^{-1}$. Since the group $F=$ $\left\{\alpha_{h}: h \in H\right\} \subset$ Aut $(D(n d))$ is finite and $\mathbb{J}^{d} \cong \theta=\left\{V_{a}: a \in A\right\} \subset D(n d) \cong \mathbb{J}^{\text {nd }}$ is a closed, connected, $F$-invariant subgroup, Lemma 4.2 implies the existence of a closed, connected, $F$-invariant subgroup $C \subset D(n d)$ such that $C \cdot \theta=D(n d)$ and $C \cap \theta$ is finite. Put $\Lambda=\left\{V_{h}: h \in H\right\} \subset U(n d), \Lambda=\Lambda \cdot D(n d) \subset U(n d)$, and note that $C$ is a normal subgroup of $\Lambda$. The quotient map $\xi: \Lambda \rightarrow \Lambda / C$ satisfies that

$$
\xi(\boldsymbol{\theta})=D(n d) / C=\theta / C=\xi(\theta) \subset \xi(\Lambda),
$$

and we define a continuous homomorphism $\omega: H \rightarrow \xi(\Lambda)$ by setting

$$
\omega(h)=\xi\left(Q_{h}\right)=\xi\left(D_{h}\right)^{-1} \xi\left(V_{h}\right)
$$

for every $h \in H$ (cf. (4.2)-(4.3)).

Put $H^{\prime}=V^{-1}\left(\xi^{-1}(\omega(H))=\left\{h \in H: V_{h} \in \omega(H)\right\}\right.$. From (4.3) and (4.4) it is clear that there exists, for every $h \in H$, an element $a \in A$ with $a h \in H^{\prime}$, i.e. that $A \cdot H^{\prime}=$ $H^{\prime} \cdot A=H$. Furthermore, since $\omega(A)=\left\{\mathbf{1}_{\wedge}\right\}$, and since $\operatorname{ker}(\xi) \cap \theta=C \cap \theta$ and $\operatorname{ker}(V) \cap A$ are both finite, the group $H^{\prime} \cap A$ is finite.

LEMMA 4.4. Let $H$ be a compact Lie group, $A \subset H$ a closed normal, abelian subgroup, and let $H^{\prime} \subset H$ be a closed, normal subgroup such that $H^{\prime} \cap A$ is finite and $H \cdot A=H$. If $\eta: H \rightarrow H / H^{\prime}=H^{\prime \prime}$ is the quotient map and $n \geq 1$, put

$$
H_{h}=\eta^{-1}\left(\left\{u \in H^{\prime \prime}: u^{n !}=1_{H^{\prime \prime}}\right\}\right) \text {. }
$$

Then $\left(H_{n}, n \geq 1\right)$ is an increasing sequence of closed, normal subgroups of $H, \cup_{n} H_{n}$ is dense in $H$, and $H_{n} \cap A$ is finite for every $n \geq 1$.

Proof. For every $n \geq 1$ we set $H^{\prime \prime}(n)=\left\{u \in H^{\prime \prime}: u^{n !}=1_{H^{\prime \prime}}\right\}$. Then $H^{\prime \prime}(n)$ is a finite subgroup of the abelian Lie group $H^{\prime \prime}$, and $\bigcup_{n} H^{\prime \prime}(n)$ is dense in $H^{\prime \prime}$. Hence $H_{n}=\eta^{-1}\left(H^{\prime \prime}(n)\right)$ has finite intersection with $A$, and $\bigcup_{n} H_{n}$ is dense in $H$.

For the remainder of this section $\Gamma$ will denote an infinite, finitely generated, abelian group.

LeMMA 4.5. Let $X$ be a compact, abelian group, $\Gamma \subset$ Aut $(X)$, and assume that $(X, \Gamma)$ satisfies the descending chain condition. For every $n \geq 1$, put $X^{(m)}=\left\{x \in X: x^{m}=1_{X}\right\}$. Then $\bigcup_{m} X^{(m)}$ is dense in $X$. 
Proof. If $X$ is abelian, Theorem 3.2 implies that $(X, \Gamma)$ is conjugate to a closed subgroup $Y$ of $\left(\mathbb{T}^{k}\right)^{\Gamma}$ for some $k \geq 1$, where $\mathbb{T}=\mathbb{R} / \mathbb{Z}$. Hence the dual group $Y^{\wedge}$ is isomorphic to a quotient group of the direct sum of infinitely many copies of $\mathbb{Z}$, and we can find a decreasing sequence $\left(V_{n}, n \geq 1\right)$ of subgroups of $\boldsymbol{Y}^{\hat{A}}$ such that $Y^{\wedge} / V_{n}$ is finite for every $n \geq 1$ and $\bigcap_{n \geq 1} V_{n}=\{0\}$. Put $A_{n}=V_{n}^{\perp} \subset X$, where $V_{n}^{\perp}$ is the annihilator subgroup of $V_{n}$. Since $\hat{A}_{n}=Y^{\wedge} / V_{n}, A_{n}$ is finite, and $\bigcup_{n} A_{n}$ is dense in $X$. For every $n \geq 1$ we can find an $m \geq 1$ such that $A_{n} \subset X^{(m)}$, and this proves that $\bigcup_{m} X^{(m)}$ is dense in $X$.

LEMMA 4.6. Let $G$ be a compact Lie group, $Y \subset G^{\prime}$ a full, shift-invariant subgroup, and let $B \subset Y$ be a closed, normal, abelian, shift invariant subgroup. Choose a finite set $D \subset \Gamma$ such that

$$
Y=\left\{y \in G^{\mathrm{r}}: \pi_{D}\left(T_{y} y\right) \in H \quad \text { for every } y \in \Gamma\right\}
$$

and

$$
B=\left\{y \in G^{\mathrm{r}}: \pi_{D}\left(T_{\gamma} y\right) \in A \quad \text { for every } \gamma \in \Gamma\right\},
$$

where $H=\pi_{D}(Y)$ and $A=\pi_{D}(B)$ (cf. Corollary 3.8). Then $A \subset H$ is a closed, normal, abelian subgroup. Let $H^{\prime} \subset H$ be chosen as in Lemma 4.3, denote by $\eta: H \rightarrow H / H^{\prime}=H^{\prime \prime}$ the quotient map, and define $\left(H_{n}, n \geq 1\right)$ by (4.5). For every $n \geq 1$ put

$$
Y_{n}=\left\{y \in G^{\Gamma}: \pi_{D}\left(T_{\gamma} y\right) \in H_{n} \quad \text { for every } \gamma \in \Gamma\right\} .
$$

Then $\left(Y_{n}, n \geq 1\right)$ is an increasing sequence of closed, normal, shift-invariant subgroups of $Y, \cup_{n} Y_{n}$ is dense in $Y$, and $Y_{n} \cap B$ is zero dimensional for all $n \geq 1$.

Proof. Consider the homomorphism $\eta: Y \rightarrow H^{\prime \prime}$ given by $\eta(y)(\gamma)=\eta\left(\pi_{D}\left(T_{\gamma} y\right)\right)$, $\gamma \in \Gamma, y \in Y$. Since $H^{\prime \prime}$ is abelian, the group $\eta(Y)=Z \subset H^{\prime \prime \Gamma}$ is abelian. Furthermore $Y_{1}=\operatorname{ker}(\eta)$, and our choice of $H^{\prime}$ implies that $Y_{1} \cap B$ is zero dimensional. Lemma 4.5 shows that the elements of finite order are dense in $Z$. Hence the subgroups $Z^{(n)}=\left\{z \in Z: z^{n}=1_{Z}\right\}, n \geq 1$, have dense union in $Z$. From (4.5) it is clear that $\eta^{-1}\left(Z^{(n)}\right) \subset Y_{n}$, and hence $\bigcup_{n} Y_{n}$ is dense in $Y$. Since $H_{n} \cap A$ is finite we also know that $Y_{n} \cap B$ is zero dimensional for every $n \geq 1$.

THEOREM 4.7. Let $X$ be a compact group, $\Gamma \subset$ Aut $(X)$ a finitely generated, abelian group, and assume that $(X, \Gamma)$ satisfies the descending chain condition. Suppose that $B \subset X$ is a closed, normal, abelian, $\Gamma$-invariant subgroup. Then there exist a compact Lie group $H$, a closed, normal, abelian subgroup $A \subset H$, an increasing sequence $\left(H_{n}, n \geq 1\right)$ of closed, normal subgroups of $H$ and a continuous conjugacy $\varphi$ of $(X, \Gamma)$ onto a full, shift invariant subgroup $Y$ of $H^{r}$ with the following properties.

(1) For every $n \geq 1, H_{n} \cdot A=H$, and $H_{n} \cap A$ is finite;

(2) if $X_{n}=\varphi^{-1}\left(Y \cap H_{n}^{\Gamma}\right), n \geq 1$, then $\left(X_{n}, n \geq 1\right)$ is an increasing sequence of closed, normal, $\Gamma$-invariant subgroups of $X$ such that $\bigcup_{n} X_{n}$ is dense in $X$, and $\varphi\left(X_{n}\right)$ is a full, shift-invariant subgroup of $H_{n}^{\Gamma}$ for every $n \geq 1$;

(3) $\varphi(B)=Y \cap A^{\mathrm{l}}$, and $\varphi(B)$ is a full subgroup of $A^{\mathrm{l}}$;

(4) for every $n \geq 1, X_{n} \cap B \subset \varphi^{-1}\left(H_{n} \cap A\right)^{r}$ and hence zero dimensional, since $H_{h} \cap A$ is finite.

Proof. Use Theorem 3.2 to find a compact Lie group $G$ and a continuous conjugacy 
$\varphi: X \rightarrow G^{\Gamma}$ of $X$ into $G^{\Gamma}$ such that $Y=\varphi(X)$ is a full, shift-invariant subgroup of $G^{\Gamma}$. We choose $D \subset \Gamma, H \subset G^{D}$, and $A \subset H$ as in Lemma 4.6 and define $\left(H_{n}, n \geq 1\right)$ by (4.5) and $\left(Y_{n}, n \geq 1\right)$ by (4.6). If $Y_{n} \subset\left(H_{n}\right)^{\Gamma}$ is not full we re-define $H_{n}$ as $H_{n}=\pi_{\left\{1_{1}\right\}}\left(Y_{n}\right), n \geq 1$, without affecting any of the other assertions. The proof is completed by setting $X_{n}=\varphi^{-1}\left(Y_{n}\right), n \geq 1$.

Corollary 4.8. Let $X$ be a compact group such that $X^{0}$ is abelian, where $X^{0}$ is the connected component of the identity in $X, \Gamma \subset$ Aut $(X)$, and assume that $(X, \Gamma)$ satisfies the descending chain condition. Then there exists an increasing sequence $\left(X_{n}, n \geq 1\right)$ of closed, normal, zero dimensional, $\Gamma$-invariant subgroups of $X$ such that $\cup_{n} X_{n}$ is dense in $X$.

Proof. Apply Theorem 4.7 to $B=X^{0}$ and observe that $A$ is open in $H, H / A$ is finite, and hence $H_{n}$ is finite and $X_{n}$ is zero dimensional for every $n \geq 1$.

COROllary 4.9. Let $X$ be a compact, connected group, $\Gamma \subset$ Aut $(X)$, and assume that $(X, \Gamma)$ satisfies the descending chain condition. Then there exists a compact, connected Lie group $H$, an increasing sequence $\left(H_{n}, n \geq 1\right)$ of closed, normal, subgroups of $H$, and a continuous conjugacy $\varphi$ of $(X, \Gamma)$ onto a full, shift invariant subgroup $Y$ of $H^{\Gamma}$ such that the following conditions are satisfied for every $n \geq 1$ :

(1) the centre $C\left(H_{n}\right)$ of $H_{n}$ is finite;

(2) $Y_{n}=Y \cap\left(H_{n}\right)^{\Gamma}$ is a full subgroup of $\left(H_{n}\right)^{\mathrm{Y}}$;

(3) if $X_{n}=\varphi^{-1}\left(Y_{n}\right), n \geq 1$, then $\bigcup_{n} X_{n}$ is dense in $X$.

Proof. Put $B=C(X)$ in Theorem 4.7 and observe that, if $H$ is a compact, connected Lie group and $K \subset H$ a closed, normal subgroup, then $C(K) \subset C(H)$.

\section{Expansive automorphisms of compact groups}

Definition 5.1. Let $X$ be a compact group, and let $\Gamma \subset$ Aut $(X)$ be a countable group. The pair $(X, \Gamma)$ is expansive (or $\Gamma$ acts expansively on $X$ ) if there exists a neighbourhood $N$ of the identity in $X$ such that $\bigcap_{\gamma \in \Gamma} \gamma(N)=\left\{\mathbf{1}_{X}\right\}$. If $G$ a compact group, and $X \subset G^{\Gamma}$ a closed, shift-invariant subgroup, then $X$ will be called expansive if the shifts $T_{1}$ act expansively on $X$.

TheOREM 5.2. Let $X$ be a compact group, $\Gamma \subset$ Aut $(X)$ a finitely generated, abelian group, and assume that $(X, \Gamma)$ is expansive. Then $(X, \Gamma)$ satisfies the descending chain condition. Conversely, if $X$ is zero dimensional and $(X, \Gamma)$ satisfies the descending chain condition then $(X, \Gamma)$ is expansive.

Proof. Since the action of $\Gamma$ on $X$ is expansive, there exists a neighbourhood $N$ of $\mathbf{1}_{X}$ with $\bigcap_{\gamma \in \Gamma^{\prime}} \gamma(N)=\left\{\mathbf{1}_{X}\right\}$. The compactness of $X$ implies the existence of a finite dimensional, continuous, unitary representation $\sigma$ of $X$ and of an $\varepsilon>0$ such that $N^{\prime}=\left\{x \in X:\left\|\sigma(x)-\sigma\left(1_{X}\right)\right\|<\varepsilon\right\} \subset N$, where $\|\cdot\|$ denotes the operator norm. We put $G=\sigma(X)$, observe that $G$ is a compact Lie group, and define a continuous, injective homomorphism $\varphi: X \rightarrow G^{\Gamma}$ by $\varphi(x)(\gamma)=\sigma(\gamma(x))$ for every $x \in X, \gamma \in \Gamma$. By Theorem 3.2, $(X, \Gamma)$ satisfies the descending chain condition. The converse follows from Proposition 3.12.

If $X$ is a compact group and $\alpha \in \operatorname{Aut}(X)$ we say that $(X, \alpha)$ is expansive or satisfies the descending chain condition if the corresponding condition holds for 
$\left(X,\left\{\alpha^{n}: n \in \mathbb{Z}\right\}\right)$. Similarly we call $(X, \alpha)$ conjugate to a shift-invariant subgroup $Y$ of $G^{\mathbb{Z}}$, where $G$ is a compact Lie group, if $\left(X,\left\{\alpha^{n}: n \in \mathbb{Z}\right\}\right)$ is conjugate to $Y$.

THEOREM 5.3. Let $X$ be a compact group and let $\alpha \in$ Aut $(X)$ be expansive. Then there exists a compact Lie group $G$ whose connected component $G^{0}$ of the identity is abelian, and such that $(X, \alpha)$ is conjugate to a full, shift-invariant subgroup of $G^{\mathbb{Z}}$. In particular, the connected component $X^{0}$ of the identity in $X$ is abelian.

The assertion that $X^{0}$ must be abelian was proven in [La, Corollary 3.3]. We shall derive Theorem 5.3 from certain properties of shift-invariant subgroups of $G^{\mathbb{Z}}$, where $G$ is a compact group. The following result shows that every automorphism $\alpha$ of a compact group $X$ which satisfies the descending chain condition can be represented as a one step Markov shift.

Proposition 5.4. Let $X$ be a compact group, $\alpha \in$ Aut $(X)$, and assume that $(X, \alpha)$ satisfies the descending chain condition. Then there exist a compact Lie group $G, a$ full subgroup $H \subset G \times G$, and a continuous conjugacy $\varphi$ of $X$ into $G^{\mathbb{Z}}$ with the following properties.

(1) $\varphi(X)=Y_{H}$, where

$$
Y_{H}=\left\{y \in G^{\mathbb{Z}}:(y(k), y(k+1)) \in H \quad \text { for every } k \in \mathbb{Z}\right\}
$$

(2) if $G^{0}, H^{0}$ and $X^{0}$ are the connected components of the identity in $G, H$, and $X$, respectively, then $H^{0}$ is a full subgroup of $G^{0} \times G^{0}$, and

$$
\varphi\left(X^{0}\right)=\left\{y \in G^{\mathbb{Z}}:(y(k), y(k+1)) \in H^{0} \quad \text { for every } k \in \mathbb{Z}\right\} .
$$

In particular, $X$ is connected if and only if $H$ is connected.

Proof. This is a consequence of the Corollaries 3.9 and 3.11, applied to the groups $X$ and $X^{0}$. If $G, \varphi$ and $H \subset G \times G$ satisfy $(5.1)$ then $\varphi\left(X^{0}\right) \subset\left(G^{0}\right)^{\mathbb{Z}}$. Since $X / X^{0}$ is zero dimensional and $G$ and $H$ are Lie groups, $\pi_{\{0\}}\left(\varphi\left(X^{0}\right)\right)$ and $\pi_{\{0,1\}}\left(\varphi\left(x^{0}\right)\right)$ are open subgroups of $G^{0}$ and $H^{0}$, respectively, hence $\pi_{\{0\}}\left(\varphi\left(X^{0}\right)\right)=G^{0}$, and $\pi_{\{0,1\}}\left(\varphi\left(X^{0}\right)\right)=H^{0}$ is a full subgroup of $G^{0} \times G^{0}$. This proves (5.2).

Definition 5.5. Let $G$ be a compact group. A full, shift-invariant subgroup $X \subset G^{\mathbb{Z}}$ is called a Markov subgroup if there exists a (necessarily full) subgroup $H \subset G \times G$ such that $X=Y_{H}$, where $Y_{H}$ is defined by (5.1).

Proposition 5.4 implies in particular that every expansive automorphism of a compact group $X$ is conjugate to the shift on a Markov subgroup of $G^{\mathbb{Z}}$, where $G$ is a compact Lie group. For the following lemma we fix a compact group $G$ (not necessarily Lie) and a Markov subgroup $Y_{H} \subset G^{\mathbb{Z}}$, where $H \subset G \times G$ is a full subgroup (cf. (5.1)). For every $g \in G$ and $n \in \mathbb{Z}$, put

$$
F_{H}(g, n)=\left\{y(n): y \in Y_{H} \text { and } y(0)=g\right\} \subset G
$$

and

$$
F_{H}(n)=F_{H}\left(\mathbf{1}_{G}, n\right) \text {. }
$$

Then $F_{H}(n)$ is a closed, normal subgroup of $G$ with

$$
F_{H}(n) \subset F_{H}(n+1) \text { and } F_{H}(-n) \subset F_{H}(-n-1)
$$


for every $n \geq 0$, since $\left(1_{G}, 1_{G}\right) \in H$. Note that $F_{H}(g, n), n \geq 1$, are the usual follower sets of symbolic dynamics, and $F_{H}(g, n), n \leq-1$, are the predecessor sets. From the definition of $F_{H}(g, n)$ it is clear that the map $\theta_{n}^{\prime}: G \rightarrow G / F_{h}(n)$, obtained by setting

$$
\theta_{n}^{\prime}(g)=F_{H}(g, n) / F_{H}(n),
$$

is a well defined, continuous group homomorphism with kernel $F_{H}(-n)$ and induces a continuous isomorphism

$$
\theta_{n}: G / F_{H}(-n) \rightarrow G / F_{H}(n) .
$$

The next lemma (and its proof) is largely contained in Lemma 4 in [MT] (cf. also [Ki]). The reason for presenting it in such detail is that it provides one of the main steps in many of the later proofs in this paper, where the precise identification (in terms of predecessor and follower sets) of the various subgroups and maps involved will be of crucial importance.

LEMMA 5.6. Put $\Lambda=F_{H}(-1) \cap F_{H}(1), \quad G^{\prime}=G / F_{H}(1), \quad H^{\prime}=$ $H /\left(F_{H}(1) \times F_{H}(1)\right) \subset G^{\prime} \times G^{\prime}$, and denote by $\eta: G \rightarrow G^{\prime}$ the quotient map. The shift commuting homomorphism $\eta: Y_{H} \rightarrow G^{\prime \mathbb{Z}}$ given by $\eta(y)(n)=\eta(y(n)), n \in \mathbb{Z}, y \in Y_{H}$, has the following properties.

$$
\boldsymbol{\eta}\left(Y_{H}\right)=Y_{H^{\prime}}
$$

where $Y_{H^{\prime}} \subset G^{\prime \mathbb{Z}}$ is defined by (5.1) with $H^{\prime}$ replacing $H$.

$$
Y_{H} \cap F_{H}(1)^{\mathbb{Z}}=\operatorname{ker}(\eta)=\Lambda^{\mathbb{Z}} \text {. }
$$

(3) If $F_{H^{\prime}}(n), n \in \mathbb{Z}$, is defined by (5.4) with $Y_{H^{\prime}} \subset G^{\prime \mathbb{Z}}$ replacing $Y_{H}$, then

$$
F_{H} \cdot(n)=F_{H}(n+1) / F_{H}(1)
$$

for every $n \geq 1$.

(4) There exists a Borel isomorphism $\varphi: Y_{H} \rightarrow \Lambda^{\mathbb{Z}} \times Y_{H^{\prime}}$ which carries the shift on $Y_{H}$ to the cartesian product of the shifts on $\Lambda^{\mathbb{Z}}$ and $Y_{H^{\prime}}$.

(5) If $G$ is finite the map $\varphi: Y_{H} \rightarrow \Lambda^{\mathbb{Z}} \times Y_{H^{\prime}}$ in (4) can be chosen to be a homeomorphism.

(6) If $Y_{H}$ is expansive then $\Lambda$ is finite and $Y_{H^{\prime}}$ is again expansive.

Proof. From the definition of $F_{H}( \pm 1)$ it is clear that $h \in F_{H}(1)\left(h \in F_{H}(-1)\right)$ if and only if $\left(\mathbf{1}_{G}, h\right) \in H\left(\left(h, \mathbf{1}_{G}\right) \in H\right)$. In particular, if $\eta^{\prime}: H \rightarrow H^{\prime}$ denotes the quotient map, and if $(g, h) \in H$ and $\eta^{\prime}(g, h)=(\eta(g), \eta(h))=(u, v) \in H^{\prime}$, then $(g, h k) \in H$ and $\eta^{\prime}(g, h k)=(u, v)$ for every $k \in F_{H}(1)$. It follows that there exists, for every $(u, v) \in H^{\prime}$, and for every $h \in G$ with $\eta(h)=v$, an element $g \in G$ with $\eta(g)=u$ and $(g, h) \in H$. This implies (5.8).

Now assume that $(g, h) \in \operatorname{ker}\left(\eta^{\prime}\right)$. Then $g, h \in F_{H}(1)$, i.e. $\left\{\left(1_{G}, h\right),\left(g, 1_{G}\right)\right\} \subset$ $\operatorname{ker}\left(\eta^{\prime}\right) \subset H$. In particular, $g \in F_{H}(-1) \cap F_{H}(1)$. This shows that $y \in \operatorname{ker}(\eta) \subset Y_{H}$ if and only if $y(n) \in \Lambda$ for all $n \in \mathbb{Z}$. From the definition of $Y_{H}$ it is clear that $\Lambda^{\mathbb{Z}} \subset Y_{H}$. We have proved (5.9), and (5.10) follows from the definition of $F_{H}(n), n \geq 1$.

We denote by $\tau: G \rightarrow G / \Lambda$ the quotient map, choose a Borel map $\omega: G / \Lambda \rightarrow G$ with $\omega\left(\mathbf{1}_{G / \Lambda}\right)=1_{G}$ and $\tau \cdot \omega=\mathrm{id}_{G / \Lambda}$, and define a Borel map $\omega:(G / \Lambda)^{\mathbb{Z}} \rightarrow Y_{H} \subset G^{\mathbb{Z}}$ by $\omega(u)(k)=\omega(u(k)), k \in \mathbb{Z}, u \in(G / \Lambda)^{\mathbb{Z}}$. Equation (5.9) shows that the continuous homomorphism $\tau: Y_{H} \rightarrow(G / \Lambda)^{\mathbb{Z}}$ with $\tau(y)(k)=\tau(y(k)), k \in \mathbb{Z}, y \in Y_{H}$, has the 
property that $\operatorname{ker}(\tau)=\operatorname{ker}(\boldsymbol{\eta})$. It follows that map $\boldsymbol{\theta}: Y \rightarrow \Lambda^{\mathbb{Z}} \times Y_{H^{\prime}}$, given by $\boldsymbol{\theta}(\boldsymbol{y})=$ $\left(y \cdot \boldsymbol{\omega}(\boldsymbol{\tau}(y))^{-1}, \boldsymbol{\eta}(y)\right), y \in Y$, is a Borel isomorphism, and that $\boldsymbol{\theta} \cdot T_{1}=\left(T_{1} \times T_{1}^{\prime}\right) \cdot \boldsymbol{\theta}$, where $T_{1}^{\prime}$ is the shift on $Y_{H^{\prime}}$. If $G$ is finite, then $\omega$ and $\omega$ are both continuous, and we have proved (4) and (6).

If $Y_{H}$ is expansive, then $\Lambda^{\mathbb{Z}} \subset Y_{H}$ is again expansive, which implies that $\Lambda$ is finite. We write $\rho: G \rightarrow G / \Lambda$ for the quotient map and observe that $\rho$ is a homeomorphism of a neighbourhood $N$ of the identity in $G$ onto a neighbourhood $N^{\prime}$ of the identity in $G / \Lambda$. By decreasing $N$, if necessary, we may assume that $Y_{H} \cap N^{\mathbb{Z}}=$ $\left\{\mathbf{1}_{G^{\mathbf{z}}}\right\}$ (the existence of such a neighbourhood $N \subset G$ is equivalent to the expansiveness of $\left.Y_{H}\right)$. Define a shift commuting homomorphism $\rho: Y_{H} \rightarrow(G / \Lambda)^{\mathbb{Z}}$ by $\rho(y)(n)=$ $\rho(y(n)), n \in \mathbb{Z}$, and put $Y^{\prime}=\rho\left(Y_{H}\right)$. If $Y^{\prime}$ is not expansive there exists a point $u \in Y^{\prime} \cap N^{\prime \mathbb{Z}}$ such that $u \neq \mathbf{1}_{(G / \Lambda)^{2}}$. If $u=\rho(y)$ for some $y \in Y_{H}$ we can choose a point $z \in \Lambda^{\mathbb{Z}}$ with $u(n) z(n)^{-1} \in N$ for all $n \in \mathbb{Z}$. Since $u z^{-1} \in Y_{H} \cap N^{\mathbb{Z}}$, but $u z^{-1} \neq \mathbf{1}_{G^{\mathbb{Z}}}$ we have arrived at a contradiction. Hence $Y^{\prime}$ is expansive. The continuous homomorphism $\eta: Y_{H} \rightarrow G^{\prime \mathbb{Z}}$ has kernel $\Lambda^{\mathbb{Z}}$ and therefore induces a continuous, shift commuting isomorphism $\eta^{\prime}: Y^{\prime}=Y / \Lambda^{\mathbb{Z}} \rightarrow Y_{H^{\prime}}$, and the expansiveness of $Y^{\prime}$ implies that $Y_{H^{\prime}}$ is expansive. This proves (5).

Proposition 5.7. Let $G$ be a compact group and let $Y=Y_{H} \subset G^{\mathbb{Z}}$ be a Markov subgroup. We define $F_{H}(n), n \in \mathbb{Z}$, by (5.4). For every $k \geq 0$ we set $Y_{k}=Y \cap F_{H}(k)^{\mathbb{Z}}$, $G_{k}=G / F_{H}(k)$, denote by $\eta^{(k)}: G \rightarrow G_{k}$ the quotient map, and define a shift commuting map $\boldsymbol{\eta}^{(k)}: Y \rightarrow G_{k}^{\mathbb{Z}}$ with $\operatorname{ker}\left(\boldsymbol{\eta}^{(k)}\right)=Y_{k}$ by $\boldsymbol{\eta}^{(k)}(y)(n)=\eta^{(k)}(y(n)), n \in \mathbb{Z}$. The maps $\boldsymbol{\eta}^{(k)}, k \geq 0$, have the following properties.

(1) $\eta^{(k)}(Y)=Y_{H_{k}} \subset G_{k}^{\mathbb{Z}}$, where $H_{k}=H /\left(F_{H}(k) \times F_{H}(k)\right)$, and where $Y_{H_{k}}$ is defined by (5.1) with $H_{k}$ and $G_{k}$ replacing $H$ and $G$.

(2) $Y_{k+1} / Y_{k} \cong \eta^{(k)}\left(Y_{k+1}\right)=\Lambda_{k+1}^{\mathbb{Z}}$ for some closed, normal subgroup $\Lambda_{k+1} \subset$ $F_{H}(k+1) / F_{H}(k)$.

If $G$ is a compact Lie group with finite centre then the following stronger assertions are true.

(3) There exists an integer $K \geq 1$ with $F_{H}(k)=F_{H}(K)$ for every $k \geq K$, and a continuous automorphism $\tau$ of the group $G_{K}$ such that $H_{K}=\left\{(u, \tau(u)): U \in G_{K}\right\}$. Hence

$Y / Y_{K} \cong \boldsymbol{\eta}^{(K)}(Y)=Y_{H_{K}}=\left\{v \in G_{K}^{\mathbb{Z}}: v(n+1)=\tau(v(n))\right.$ for every $\left.n \in \mathbb{Z}\right\} \cong G_{K}$,

and this isomorphism sends the shift $T_{1}$ on $\eta^{(K)}(Y)$ to the automorphism $\tau$ on $G_{K}$.

(4) There exists a Borel isomorphism

$$
\varphi: Y \rightarrow \Lambda_{1}^{\mathbb{Z}} \times \Lambda_{2}^{\mathbb{Z}} \times \cdots \times \Lambda_{K}^{\mathbb{Z}} \times G_{K}
$$

which carries $T_{1}$ to $T_{1}^{(1)} \times \cdots \times T_{1}^{(K)} \times \tau$, and $\varphi$ can be chosen to be a homeomorphism whenever $G$ is finite.

(5) The restriction of $T_{1}$ to $Y_{k}$ is ergodic, and $T_{1}$ is ergodic on $X$ if and only if $G_{K}=\{1\}$.

(6) If $Y_{H}$ is expansive, then $G$ is finite.

Proof. In order to prove (1) we apply Lemma 5.6 to see that $\boldsymbol{\eta}^{(1)}(Y)=Y_{H_{1}}$ and note that the groups $F_{H_{1}}(n), n \in \mathbb{Z}$, defined by (5.4) with $Y_{H_{1}}$ and $G_{1}$ replacing $Y_{H}$ and $G$, satisfy that $F_{H_{1}}(n)=F_{H}(n+1) / F_{H}(1)$ for all $n \geq 1$. Repeated application of 
Lemma 5.6 shows that $\eta^{(k)}(Y)=Y_{H_{k}} \subset G_{k}^{\mathbb{Z}}$ and $F_{H_{k}}(n)=F_{H}(n+k) / F_{H}(k)$ for $k$, $n \geq 1$, where $F_{H_{k}}(n)$ is again obtained from (5.4) by using $Y_{H_{k}}$ and $G_{k}$ instead of $Y_{H}$ and $G$. All the assertions in (1) are now obvious.

For every $k \geq 0$, put $\Lambda_{k+1}=F_{H_{k}}(-1) \cap F_{H_{k}}(1) \subset F_{H}(k+1) / F_{H}(k) \subset G_{k}$, where $H_{0}=H$ and $G_{0}=G$. From Lemma 5.6 we know that $\eta^{(k)}\left(Y_{k+1}\right)=\eta^{(k)}(Y) \cap F_{H_{k}}(1)^{\mathbb{Z}}=$ $\Lambda_{k+1}^{\mathbb{Z}}$, and this proves (2).

If $G$ is a compact Lie group with finite centre then $G$ has only finitely many closed, normal subgroups. In particular the sequence $\left(F_{H}(n), n \geq 1\right)$ must eventually become constant. The first part of the proof shows that $F_{H_{K}}(n)=$ $F_{H}(n+K) / F_{H}(K)=\left\{1_{G_{k}}\right\}$ for every $n \geq 1$. Since $G_{K}$ is a quotient of a compact Lie group with finite centre, $G_{K}$ again has finite centre, and we conclude that there exists an $L \geq 1$ with $F_{H_{K}}(-n)=F_{H_{K}}(-L)$ for all $n \geq L$. For every $n \geq 1$ we denote by $\theta_{n}: G_{K} / F_{H_{K}}(-n) \rightarrow G_{K} / F_{H_{K}}(n)=G_{K}$ the isomorphism described in (5.7) and put $\tau=\theta_{L+1} \theta_{L}^{-1}: G_{K} \rightarrow G_{K}$. From the definition of $\tau$ it is clear that $H_{K}=$ $\left\{(u, \tau(u)): u \in G_{K}\right\}$, and the other statements in (3) are immediate consequences of this.

Repeated application of (4) and (5) in Lemma 5.6 yields (4), and (5) is obvious from (4), since $G_{K}$ has no ergodic automorphisms. Finally, if $Y$ is expansive, Lemma 5.6(6) implies that $Y_{H_{k}}$ is expansive and $\Lambda_{k}$ is finite for $k=1, \ldots, K$. In particular, $Y_{H_{K}}=\left\{v \in G_{K}^{\mathbb{Z}}: v(n+1)=\tau(v(n))\right.$ for all $\left.n \in \mathbb{Z}\right\}$ is expansive. Since $G_{K}$ is a compact Lie group with finite centre, Aut $\left(G_{K}\right)$ is compact, and hence there exists a metric $d$ on $G_{K}$ which is invariant under Aut $\left(G_{K}\right)$ and in particular under $\tau$. For every $\varepsilon>0$, denote $B(\varepsilon) \subset G_{K}$ the $\varepsilon$-ball in the metric $d$ centred at $\mathbf{1}_{G_{K}}$. If $G_{K}$ is not finite, there exists, for every $\varepsilon>0$, a point $\mathbf{1}_{G_{K}} \neq U_{\varepsilon} \in B(\varepsilon)$, and we define $V_{F} \in Y_{H_{k}} \cap$ $B(\varepsilon)^{\mathbb{Z}}$ by setting $V_{\varepsilon}(n)=\tau^{n}\left(u_{\varepsilon}\right)$ for every $n \in \mathbb{Z}$. This contradicts the expansiveness of $Y_{H_{K}}$, and we conclude that $G_{K}$ must be finite. From (1)-(3) se see that $Y$ is zero dimensional, and Proposition 3.12 shows that $G$ is finite.

Corollary 5.8. (cf. [Ki] and Theorem A in [MT].) Let $X$ be a compact, zero dimensional group, and let $\alpha \in \operatorname{Aut}(X)$ be an expansive automorphism. Then there exist finite groups $\Lambda_{1}, \ldots, \Lambda_{K}, G_{K}$, an automorphism $\tau$ of $G_{K}$, and a homeomorphism $\varphi: X \rightarrow \Lambda_{1}^{\mathbb{Z}} \times \cdots \times \Lambda_{K}^{\mathbb{Z}} \times G_{K}$ which carries $\alpha$ to $T_{1}^{(1)} \times \cdots \times T_{1}^{(K)} \times \tau$, where $T_{1}^{(i)}$ denotes the shift on $\Lambda_{i}^{\mathbb{Z}}$. The automorphism $\alpha$ is ergodic if and only if $G_{K}=\{1\}$.

Proof. Theorem 5.2 and Proposition 3.12 allow us to regard $X$ as a Markov subgroup $Y=Y_{H} \subset G^{\mathbb{Z}}$, where $G$ is finite. Now apply Proposition 5.7(4) and (5).

Proof of Theorem 5.3. We have to show that $X^{0}$ is abelian. Assume for simplicity that $X=X^{0}$ and that $\alpha$ is expansive. By Theorem $5.2(X, \alpha)$ satisfies the descending chain condition. We apply Corollary 4.9 and choose a compact, connected Lie group $G$, an increasing sequence $\left(G_{n}, n \geq 1\right)$ of closed, normal subgroups of $G$ with finite centres such that $\bigcup_{n} G_{n}$ is dense in $G$, and a continuous conjugacy $\varphi$ of $(X, \alpha)$ onto a full, shift invariant subgroup $Y \subset G^{\mathbb{Z}}$ such that $\bigcup_{n} X_{n}$ is dense in $X$, where $X_{n}=\varphi^{-1}\left(Y_{n}\right)$ and $Y_{n}=Y \cap G_{n}^{\mathbb{Z}}$. Then $Y_{n}$ is expansive, and Proposition 5.4 and Proposition $5.7(6)$ together imply that $G_{n}$ is finite for every $n \geq 1$. Since $G$ is 
connected and $G_{n}$ is normal in $G$ we conclude that $G_{n} \subset C(G)$, and the density of $\cup_{n} G_{n}$ in $G$ implies that $G$ is abelian.

Proposition 5.9. Let $X$ be a compact group, and let $\alpha \in$ Aut $(X)$. Denote by $X^{0}$ the connected component of the identity in $X$ and write $\alpha_{0}$ and $\alpha^{\prime}$ for the automorphisms induced by $\alpha$ on $X^{0}$ and $X / X^{0}$, respectively. Then there exists a homeomorphism $\psi: X \rightarrow X^{0} \times X / X^{0}$ such that $\psi \cdot \alpha \cdot \psi^{-1}=\alpha_{0} \times \alpha^{\prime}$.

Proof. First assume that $(X, \alpha)$ satisfies the descending chain condition. We apply Proposition 5.4, use the notation employed there, and assume for simplicity that $X=Y_{H}$ and that $\alpha=T_{1}$ (cf. (5.1)). We can find a homeomorphism of $G$ onto $G^{0} \times G / G^{0}$ which acts as the identity on $G^{0}$, and this homeomorphism induces a homeomorphism of $H$ and $H^{0} \times H / H^{0}$, since $H^{0}=H \cap\left(G^{0} \times G^{0}\right)$. From Proposition 5.4(2) we see that $Y_{H}$ is homeomorphic to $Y^{0} \times Y_{H} / Y^{0}$, and this homeomorphism conjugates $T_{1}$ in the required manner.

If $(X, \alpha)$ does not satisfy the descending chain condition, Theorem 3.16 implies the existence of a decreasing sequence $(V(n), n \geq 1)$ of closed, normal, $\alpha$-invariant subgroups of $X$ such that $\bigcap_{n} V(n)=\left\{\mathbf{1}_{X}\right\}$ and $\left(X / V(n), \alpha_{X / V(n)}\right)$ satisfies the descending chain condition for every $n \geq 1$. For every $n \geq 1, X / V(n)$ is homeomorphic to $X^{0} / V(n) \times[X / V(n)] /\left[X^{0} / V(n)\right]$ in the manner just described, and it is not difficult to verify that this splitting can be chosen consistently and therefore extends to the projective limit $(X, \alpha)$.

Corollary 5.10. Let $X$ be a compact group, and let $\alpha \in$ Aut $(X)$ be ergodic. Then $\alpha_{0}$ is ergodic on $X^{0}$.

Remark 5.11. The last statement in Theorem 5.3 generalizes a result in [ $\mathbf{W u}$ ].

\section{The structure of expansive automorphisms of compact groups}

Let $X$ be a zero dimensional compact group, and let $\alpha$ be a continuous automorphism of $X$ such that $(X, \alpha)$ satisfies the descending chain condition. From Theorem 5.2 we know that $\alpha$ is expansive. In [Ki] the first named author proved that the dynamical system $(X, \alpha)$ is topologically conjugate to one of the following three models, depending on the entropy $h(\alpha)$ and the topological transitivity of $\alpha$ (cf. Corollary 5.8):

(1) if $h(\alpha)=0$ then $\alpha$ is an automorphism of a finite group $X$;

(2) if $h(\alpha)>0$ and $\alpha$ is topologically transitive (or ergodic) then $h(\alpha)=\log m$ for some $m \geq 2$, and $(X, \alpha)$ is topologically conjugate to a full $m$-shift;

(3) if $h(\alpha)>0$ and $\alpha$ is not topologically transitive then $(\mathrm{X}, \alpha)$ is topologically conjugate to the direct product of a full $m$-shift and an automorphism of a finite group.

We refer to [Ki] for examples. If the group $X$ is connected we obtain a much more intricate class of automorphisms. The first description of an expansive automorphism of a compact, connected group $X$ (which has to be abelian by 
Theorem 5.3) was given by Williams [W1] in 1955, and Reddy [Re] gave examples of expansive automorphisms of finite dimensional tori in 1965. Later studies of expansive automorphisms of compact, connected abelian groups are due to Lawton [Ln], Aoki and Dateyama [AD], Aoki [Ao], Miles and Thomas [MT], Wilson [Ws], Arov [Ar] and Yuzvinskii [Yu].

When describing the general form of an automorphism $\alpha$ of a compact,connected, abelian group $X$ such that $(X, \alpha)$ satisfies the descending chain condition, Proposition 5.4 allows us to assume that

$$
X=Y_{H}=\left\{x \in\left(\mathbb{T}^{n}\right)^{\mathbb{Z}}:\left(x_{k}, x_{k+1}\right) \in H \text { for every } k \in \mathbb{Z}\right\},
$$

and that $\alpha=T_{1}$ (the shift on $X$ ), where $n \geq 1$ and $H \subset \mathbb{T}^{n} \times \mathbb{T}^{n}$ is a full, connected subgroup. If $h(\alpha)<\infty$ or, equivalently, if the topological dimension of $Y_{H}$ is finite, the two coordinate projections $\pi_{i}: H \rightarrow \mathbb{T}^{n}, i=1,2$, must be finite-to-one. In this case an elementary argument shows that there exists a unique matrix $A \in G L(n, \mathbb{Q})$ such that $H$ is the image of the subspace $L_{A}=\left\{(v, A v): v \in \mathbb{R}^{n}\right\} \subset \mathbb{R}^{n} \times \mathbb{R}^{n}$ under the quotient map $\kappa: \mathbb{R}^{n} \times \mathbb{R}^{n} \rightarrow \mathbb{J}^{n} \times \mathbb{J}^{n}$. Conversely, if $A \in G L(n, \mathbb{Q})$, define $L_{A}$ as above, and set

$$
H(A)=\kappa\left(\left\{(v, A v): v \in \mathbb{R}^{n}\right\}\right) .
$$

Then $H(A)$ is an $n$-dimensional subtorus of $\mathbb{T}^{n} \times \mathbb{T}^{n}$, and the two projection maps $\pi_{i}: H \rightarrow \mathbb{T}^{n}, i=1,2$, are finite-to-one. We denote by $Y_{H(A)} \subset\left(\mathbb{J}^{n}\right)^{\mathbb{Z}}$ the group defined by (6.1) with $H=H(A)$. By combining this discussion with Theorem 5.3 we have proved the following theorem.

THEOREM 6.1. Suppose that either of the following two conditions is satisfied:

(1) $X$ is a compact, connected group and $\alpha \in$ Aut $(X)$ is expansive, or

(2) $X$ is a compact, connected, abelian group, and $\alpha \in$ Aut $(X)$ is an automorphism with finite entropy such that $(X, \alpha)$ satisfies the descending chain condition.

Then there exists an integer $n \geq 1$, a matrix $A \in G L(n, \mathbb{Q})$, and a continuous isomorphism $\varphi: X \rightarrow Y_{H(A)}$ such that $\varphi(\alpha(x))=T^{A} \varphi(x)$, where $T^{A}$ denotes the shift on the group $Y_{H(A)}$ defined by (6.1) and (6.2).

The assertion of Theorem 6.1 under assumption (1) is contained in [Ln], as is our next result.

Proposition 6.2. Let $n \geq 1, A \in G L(n, \mathbb{Q})$, and let $H(A)$ and $Y_{H(A)}$, be defined by (6.1) and (6.2). The shift $T=T^{A}$ is topologically transitive (or, equivalently, ergodic) on $X=Y_{H(A)}$ if and only if $A$ has no eigenvalues which are unit roots, and $T$ is expansive if and only if $A$ has no eigenvalues of modulus 1.

Proof. In order to prove the proposition we have to compute the action of $\alpha \hat{\text { on }}$ the dual group $X^{\wedge}$ of $X$. Put

$$
\mathbb{Z}^{n}\left[A^{\prime},\left(A^{\prime}\right)^{-1}\right]=\left\langle\bigcup_{m \in \mathbb{Z}}\left(A^{\prime}\right)^{m} \mathbb{Z}^{n}\right\rangle \subset \mathbb{Q}^{n},
$$


where $\left\langle\bigcup_{m \in \mathbb{Z}}\left(A^{\prime}\right)^{m} \mathbb{Z}^{n}\right\rangle$ denotes the subgroup of $\mathbb{Q}^{n}$ generated by $\bigcup_{m \in \mathbb{Z}}\left(A^{\prime}\right)^{m} \mathbb{Z}^{n}$, furnished with the discrete topology. Let $\Xi=\sum_{\mathbb{Z}} \mathbb{Z}^{n}$ denote the dual group of $\left(\mathbb{T}^{n}\right)^{\mathbb{Z}}$. The annihilator $X^{\perp} \subset \Xi$ of $X \subset\left(\mathbb{J}^{n}\right)^{\mathbb{Z}}$ is generated by

$$
\left\{\mathbf{k}=\left(\ldots, k_{-1}, k_{0}, k_{1}, \ldots\right) \in \Xi: l \in \mathbb{Z}, d k_{l}=-B^{T} k_{l+1} \text {, and } k_{i}=0 \text { for } i \notin\{l, l+1\}\right\} \text {, }
$$

where $d \geq 1$ is an integer such that $B=d \cdot A$ has integer entries. Now consider the homomorphism $\psi: \Xi \rightarrow \mathbb{Z}^{n}\left[A^{\prime},\left(A^{\prime}\right)^{-1}\right]$ defined by

$$
\psi(\mathbf{k})=\sum_{m \in \mathbb{Z}}\left(A^{t}\right)^{m} k_{m}
$$

for every $\mathbf{k}=\left(\ldots, k_{-1}, k_{0}, k_{1}, \ldots\right) \in \Xi$. It is clear that $\psi$ is well defined, and that $\operatorname{ker}(\psi)=X^{\perp}$. Hence $\psi$ defines an isomorphism $\eta$ of $X^{\wedge}=\Xi / X^{\perp}$ onto $\mathbb{Z}^{n}\left[A, A^{-1}\right]$, and

$$
\eta\left(\hat{T^{\prime} \chi}\right)=\hat{A}^{\prime} \eta(\chi) \text { for every } \chi \in \hat{\chi},
$$

where $T^{\wedge}$ is the automorphism of $X^{\wedge}$ dual to the shift $T$ on $X$. We may thus assume that

$$
X^{\wedge}=\mathbb{Z}^{n}\left[A^{\prime},\left(A^{t}\right)^{-1}\right]
$$

and that $T^{\wedge}$ is multiplication by $A^{t}$ on $\mathbb{Z}^{n}\left[A^{\prime},\left(A^{t}\right)^{-1}\right]$. The first assertion in Proposition 6.2 is now obvious: $T$ is ergodic if and only if $A^{t}$ has no finite orbits on $\mathbb{Z}^{n}\left[A^{t},\left(A^{t}\right)^{-1}\right]$ other than $\{0\}$, i.e. if and only if $A^{t}$ - and hence $A$ - has no unit roots as eigenvalues. The second assertion is Theorem 21 in [Ln] (the equivalence of topological transitivity and ergodicity is proved in Theorem 2.1 ).

When $\alpha$ is expansive it has local product structure, shadowing, and hence Markov partitions (cf. [Bo] and [Fr]). The classification of toral automorphisms by topological conjugacy is achieved by observing that the action of a toral automorphism given by a matrix $A \in G L(n, \mathbb{Z})$ on the first homotopy group of $\mathbb{T}^{n}$ is isomorphic to the action of $A^{t}$ on $\mathbb{Z}^{n}$. Hence two such matrices $A, A^{\prime}$ induce topologically conjugate automorphisms of $\mathbb{T}^{n}$ if and only $A$ and $A^{\prime}$ are similar over $\mathbb{Z}$, i.e. if $A$ and $A^{\prime}$ lie in the same conjugacy class of $G L(n, \mathbb{Z})$. In fact, any topological conjugacy of two ergodic toral automorphisms is given by an algebraic isomorphism of the underlying tori (cf. [AP]). Arov has obtained an analogous result for automorphisms $T^{A}$ of $Y_{H(A)}$. In this context the action of $A^{t}$ on $\mathbb{Z}^{n}$ can be replaced by the action of $A$ on the Čech homology group $H_{1}\left(Y_{H(A)}, \mathbb{T}\right)$.

LEMMA 6.3. $H_{1}\left(Y_{H(A)}, \mathbb{T}\right) \cong Y_{H(A)}$, and the automorphism induced by $T^{A}$ on $H_{1}\left(Y_{H(A)}, \mathbb{T}\right)$ is equal to $T^{A}$.

Proof. For every $k \geq 0$, the group $\Omega_{k}=\left(A^{\prime}\right)^{-k} \mathbb{Z}^{n}+\cdots+\mathbb{Z}^{n}+\cdots+\left(A^{t}\right)^{k} \mathbb{Z}^{n}$ is a finitely generated subgroup of $\mathbb{Q}^{n}$ and hence isomorphic to $\mathbb{Z}^{n}$. The map $A^{\prime}: \Omega_{k} \rightarrow \Omega_{k+1}$ is injective, and $\mathbb{Z}^{n}\left[A^{\prime},\left(A^{t}\right)^{-1}\right]$ is equal to the direct limit

$$
\Omega_{1} \stackrel{A^{T}}{\longrightarrow} \Omega_{2} \stackrel{A^{T}}{\longrightarrow} \Omega_{3} \stackrel{A^{T}}{\longrightarrow} \cdots .
$$

We obtain a corresponding inverse limit for the dual group $Y_{H(A)}$ of $\mathbb{Z}^{n}\left[A^{t},\left(A^{\prime}\right)^{-1}\right]$ :

$$
X_{1} \stackrel{A}{\longleftarrow} X_{2} \stackrel{A}{\longleftarrow} X_{3} \stackrel{A}{\longleftarrow} \cdots,
$$


where each $X_{k}=\Omega_{k}{ }^{\wedge} \cong \mathbb{T}^{n}$. Since $H_{1}\left(\mathbb{T}^{n}, \mathbb{T}\right) \cong \mathbb{T}^{n}$ (cf. [ES]), we conclude from (6.4) and (6.5) that $H_{1}\left(Y_{H(A)}, \mathbb{T}\right) \cong Y_{H(A)}$. The automorphism $\left(T^{A}\right)^{\wedge}$ of $\mathbb{Z}^{n}\left[A^{t},\left(A^{\prime}\right)^{-1}\right]$ dual to $T^{A}$ corresponds to multiplication by $A^{t}$ in the direct limit (6.4). Hence $T^{A}$ corresponds to the map $\mathrm{A}$ in the projective limit (6.5), and the automorphism induced by $T^{A}$ on $H_{1}\left(Y_{H(A)}, \mathbb{J}\right)$ is equal to $T^{A}$.

THEOREM 6.4. Let $A \in G L(m, \mathbb{Q}), A^{\prime} \in G L(n, \mathbb{Q})$, and consider the shifts $T^{A}$ and $T^{A^{\prime}}$ on $Y_{H(A)}$ and $Y_{H\left(A^{*}\right)}$, respectively. The following conditions are equivalent.

(1) $\left(Y_{H(A)}, T^{A}\right)$ and $\left(Y_{H\left(A^{\prime}\right)}, T^{A^{\prime}}\right)$ are topologically conjugate;

(2) $m=n$ and there exists a continuous, shift commuting group isomorphism $\varphi: Y_{H(A)} \rightarrow$ $Y_{H\left(A^{\prime}\right)}$

(3) $m=n$ and there exists a group isomorphism $\psi^{\wedge}: \mathbb{Z}^{n}\left[A^{\prime \prime},\left(A^{\prime \prime}\right)^{-1}\right] \rightarrow \mathbb{Z}^{m}\left[A^{\prime},\left(A^{t}\right)^{-1}\right]$ such that $\hat{\psi^{\wedge} \cdot A^{\prime \prime}}=A^{t} \cdot \hat{\psi}$.

Proof. For $A \in G L(m, \mathbb{Z})$ this result is due to Adler and Palais (cf. [AP]), and for $A \in G L(m, \mathbb{Q})$, the equivalence of (1) and (2) was proved by Arov. The implications $(3) \Leftrightarrow(2) \Rightarrow(1)$ are obvious. By Lemma 6.3, any homeomorphism $\chi: Y_{H(A)} \rightarrow Y_{H\left(A^{\prime}\right)}$ with $\chi \cdot T^{A}=T^{A^{\prime}} \cdot \chi$ induces a group isomorphism $\psi: H_{1}\left(Y_{H(A)}, \mathbb{T}\right) \rightarrow H_{1}\left(Y_{H\left(A^{\prime}\right)}, \mathbb{T}\right)$ such that $\psi \cdot T^{A}=T^{A^{\prime}} \cdot \psi$. The dual isomorphism $\psi \hat{*}: \mathbb{Z}^{n}\left[A^{\prime \prime},\left(A^{\prime \prime}\right)^{-1}\right] \rightarrow$ $\mathbb{Z}^{m}\left[A^{\prime},\left(A^{\prime}\right)^{-1}\right]$ satisfies that $\psi^{\hat{\prime}} \cdot A^{\prime \prime}=A^{\prime} \cdot \hat{\psi}$, as claimed in (3). The groups $\mathbb{Z}^{m}\left[A^{\prime},\left(A^{t}\right)^{-1}\right] \subset \mathbb{Q}^{m}$ and $\mathbb{Z}^{m}\left[A^{\prime \prime},\left(A^{\prime \prime}\right)^{-1}\right] \subset \mathbb{Q}^{n}$ have dimension $m$ and $n$, respectively, over $\mathbb{Q}$. Since $\mathbb{Z}^{m}\left[A^{\prime},\left(A^{t}\right)^{-1}\right]$ and $\mathbb{Z}^{n}\left[A^{\prime \prime},\left(A^{\prime \prime}\right)^{-1}\right]$ are isomorphic as groups we conclude that $m=n$. This proves that $(1) \Rightarrow(3)$.

Remarks 6.5. (1) For every $A \in G L(n, \mathbb{Q})$, the path-connected component of the indentity in $Y_{H(A)}$ is an immersed copy of $\mathbb{R}^{k} \times \mathbb{T}^{n-k}$ for some $k$ with $0 \leq k \leq n$ : the dual group $\mathbb{Z}^{n}\left[A^{\prime},\left(A^{\prime}\right)^{-1}\right] \subset \mathbb{Q}^{n} \subset \mathbb{R}^{n}$ spans $\mathbb{R}^{n}$, hence there exists a homomorphism from $\mathbb{R}^{n}$ into $Y_{H(A)}$ whose kernel is discrete in $\mathbb{R}^{n}$, and the integer $n$ is clearly maximal with respect to this property. It is not difficult to check that the topological dimension of $Y_{H(A)}$ is, in fact, equal to $n$ (the homological dimension of $Y_{H(A)}$ is equal to $n$ by Lemma 6.3).

(2) The (topological or metric) entropy $h\left(T^{A}\right)$ of $T^{A}$ has been computed by Abramov [Ab], Arov [Ar], and Yuzvinskii [Yu]: if $P(x)=x^{n}+\alpha_{n-1} x^{n-1}+\cdots+\alpha_{0}$ is characteristic polynomial of the matrix $A \in G L(n, \mathbb{Q})$ then

$$
h\left(T^{A}\right)=\log s+\sum_{|\lambda(i)|>1} \log |\lambda(i)|,
$$

where $s$ is the least common denominator of $\left\{\alpha_{0}, \ldots, \alpha_{n-1}\right\}$ and $\{\lambda(1), \ldots, \lambda(n)\}$ is the set of eigenvalues of $\boldsymbol{A}$. This computation follows easily if we know that $T^{A}$ has Markov partitions: the entropy $h\left(T^{A}\right)$ is the growth rate of the periodic points which can easily be computed. The same formula holds, however, for the entropy $h(\alpha)$ of the automorphism $\alpha$ defined by $A$ on the group $X=\left(\mathbb{Q}^{n}\right)^{\wedge}$, although $\alpha$ will in general have no periodic points other than the identity (cf. Example 7.7(1)). A recent proof of Yuzvinskii's formula can be found in [LW].

(3) According to Theorem 6.4, two automorphisms $T^{A}$ and $T^{B}$ arising from matrices $A, B \in G L(n, \mathbb{Q})$ are topologically conjugate if and only if there exists a 
group isomorphism $\psi^{\wedge}: \mathbb{Z}^{n}\left[A^{\prime},\left(A^{\prime}\right)^{-1}\right] \rightarrow \mathbb{Z}^{n}\left[B^{T},\left(B^{T}\right)^{-1}\right]$ such that $\psi^{\wedge} \cdot A^{t}=B^{T} \cdot \psi^{\wedge}$. This problem has been shown to be algorithmically decidable by Grunewald and Segal [GS].

(4) As we have seen in (6.5), the group $Y_{H(A)}$ is a finite dimensional, projective limit of tori, i.e. a generalized solenoid (it may, of course, degenerate to a torus, as Example 6.6(1) shows).

(5) Arov has proved that, if $A, A^{\prime} \in G L(m, \mathbb{Q})$ have no eigenvalues of modulus one, every topological conjugacy between $\left(Y_{H(A)}, T^{A}\right)$ and $\left(Y_{H\left(A^{\prime}\right)}, T^{A^{\prime}}\right)$ is given by a continuous group isomorphism of $Y_{H(A)}$ and $Y_{H\left(A^{\prime}\right)}$. This can also be verified by using Theorem 6.4 and the argument in $[\mathbf{A P}]$.

Examples 6.6. (1) Let $A \in G L(n, \mathbb{Z})$. Then $H(A)=\left\{(s, t) \in \mathbb{T}^{n} \times \mathbb{T}^{n}: t=A s\right\}, X^{\wedge}=$ $\mathbb{Z}^{n}\left[A^{t},\left(A^{t}\right)^{-1}\right]=\mathbb{Z}^{n}, X=\mathbb{T}^{n}$, and $T^{A}$ is the toral automorphism associated with the matrix $A$.

(2) Let $A=\left[\begin{array}{ll}6 & 8 \\ 2 & 2\end{array}\right]$. Then $\mathbb{Z}^{2}\left[A^{t},\left(A^{t}\right)^{-1}\right]=\mathbb{Z}^{2}[1 / 2]$.

(3) Let $A=\left[\begin{array}{ll}3 & 4 \\ 1 & 1\end{array}\right], B=\left[\begin{array}{ll}3 & 2 \\ 2 & 1\end{array}\right]$. Both matrices lie in $G L(2, \mathbb{Z})$, so topological conjugacy is the same as similarity over $\mathbb{Z}$. These matrices are similar over $\mathbb{Q}$, but a simple computation shows that if $A U=U B$ and $U$ has integer entries then $\operatorname{det}(U) \in 2 \mathbb{Z}$. Hence the shifts on $Y_{H(A)}$ and $Y_{H(B)}$ are not conjugate. This example is due to Williams [W2].

(4) Let $A=\left[\begin{array}{ll}6 & 8 \\ 2 & 2\end{array}\right], B=\left[\begin{array}{ll}6 & 4 \\ 4 & 2\end{array}\right]$. As in (2), $\mathbb{Z}^{2}\left[A^{\prime},\left(A^{\prime}\right)^{-1}\right]=\mathbb{Z}^{2}\left[B^{T},\left(B^{T}\right)^{-1}\right]=\mathbb{Z}^{2}[1 / 2]$. The matrix $U=\left[\begin{array}{ll}2 & 0 \\ 0 & 1\end{array}\right]$ is an automorphism of $\mathbb{Z}^{2}[1 / 2]$ which conjugates $A$ and $B$, so the shifts $T^{A}$ and $T^{B}$ are topologically conjugate.

The following description of arbitrary, expansive automorphisms of compact groups can also be deduced from Theorem A in [MT].

TheOREM 6.7. Let $X$ be a compact group and let $\alpha \in$ Aut $(X)$ be expansive. Then $(X, \alpha)$ is topologically conjugate to a cartesian product of the form $(F, \tau) \times\left(\Sigma_{m}, T\right)$ or $(F, \tau) \times\left(\Sigma_{m}, T\right) \times\left(Y_{H(A)}, T^{A}\right)$, where

(1) $F$ is a finite group and $\tau$ an automorphism of $F$;

(2) $\Sigma_{m}$ is the full $m$-shift for some $m \geq 1$ and $T$ the shift on $\Sigma_{m}$;

(3) $n \geq 1, A \in G L(n, \mathbb{Q})$ is a matrix without eigenvalues of modulus 1 , and $Y_{M(A)}$ and $T^{A}$ are defined by (6.1) and (6.2).

Proof. By Theorem $5.2(X, \alpha)$ satisfies the descending chain condition. The assertion follows from Proposition 5.9, Theorem 6.1, Proposition 6.2, and Corollary 5.8 .

\section{Periodic points of automorphisms of compact groups}

Definition 7.1. Let $X$ be a compact group and let $\Gamma \subset$ Aut $(X)$ be a countable group. A point $x \in X$ is a periodic point of $\Gamma$ (or $\Gamma$-periodic) if $\Gamma x=\{\gamma(x): \gamma \in \Gamma\}$ is finite. If $\alpha \in$ Aut $(X)$, then $x \in X$ is a periodic point of $\alpha$ (or $\alpha$-periodic) if $\alpha^{n}(x)=x$ for some $n \geq 1$.

THEOREM 7.2. Let $X$ be a compact group such that $X^{0}$ is abelian, where $X^{0}$ is the connected component of the identity in $X$. If $\Gamma \subset$ Aut $(X)$ is a finitely generated, abelian 
group and $(X, \Gamma)$ satisfies the descending chain condition then the set of $\Gamma$-periodic points is dense in $X$.

TheOREM 7.3. [La]. Let $X$ be a compact group, and let $\Gamma \subset$ Aut $(X)$ be a finitely generated, abelian group which acts expansively on $X$. Then $X^{0}$ is abelian.

Corollary 7.4. If $\Gamma \subset$ Aut $(X)$ acts expansively on $X$ then the set of $\Gamma$-periodic points is dense in $X$.

THEOREM 7.5. Let $X$ be a compact group, and let $\Gamma \subset$ Aut $(X)$ be a finitely generated, abelian group such that $(X, \Gamma)$ satisfies the descending chain condition. If $\alpha \in \Gamma$ is ergodic then the set of $\alpha$-periodic points is dense in $X$.

Corollary 7.6. Let $X$ be a compact group, and let $\alpha \in$ Aut $(X)$ be ergodic. Suppose that there exists a finitely generated, abelian group $\Gamma \subset$ Aut $(X)$ which commutes with $\alpha$, and such that $(X, \Gamma)$ satisfies the descending chain condition. Then the set of $\alpha$-periodic points is dense in $X$.

The proofs of the Theorems 7.2, 7.3 and 7.5 will occupy following three sections. Corollary 7.4 is an immediate consequence of the Theorems 5.2, 7.2 and 7.3, and Corollary 7.6 is obvious. We illustrate these results with a few examples which show that the hypotheses in these theorems cannot be removed in general.

Examples 7.7. (1) (Furstenberg - cf. also Example 2 in [MT].) Let $X=\hat{Q} \hat{~ b e ~ t h e ~}$ solenoid, and let $\alpha \hat{}$ be the automorphism of $\mathbb{Q}$ given by $\alpha^{\wedge}(q)=q \cdot 3 / 2$ for every $q \in \mathbb{Q}$. The automorphism $\alpha$ of $X$ dual to $\alpha^{\wedge}$ has no periodic points other than the fixed point $1_{X}$. Indeed, for every $n \geq 1$, the set $\left\{x \in X: \alpha^{n}(x)=x\right\}=S_{n}$ is a closed subgroup of $X$, and its annihilator is $S_{n}^{\perp}=\left\{q \cdot\left(3^{n} / 2^{n}-1\right): q \in \mathbb{Q}\right\}=\mathbb{Q}$. Hence $S_{n}=$ $\left\{\mathbf{1}_{X}\right\}$ for every $n \geq 1$. Clearly $\mathbb{Q}$ is not finitely generated under $\hat{\alpha}$. If $Y=(\mathbb{Z}[1 / 6]) \hat{\text {, }}$ where $\mathbb{Z}[1 / 6]=\left\{k / 6^{l}: k \in \mathbb{Z}, l \geq 1\right\}$, then $\mathbb{Z}[1 / 6]$ is finitely generated under the automorphism $\alpha_{\gamma}$ induced by $\alpha$ on $Y$, and the periodic points of $\alpha_{\gamma}$ are dense by Proposition 3.13 and Theorem 7.2, or by [LP].

(2) For every $A \in G L(n, \mathbb{Q})$, the shift $T^{A}$ on $Y_{H(A)}$ defined in (6.1) and (6.2) has a dense set of periodic points by Theorem 7.2 , since $\left(Y_{H(A)}, T^{A}\right)$ satisfies the descending chain condition.

(3) Let $X=\operatorname{SU}(2)$, and let $h \in X$ be an element of infinite order (i.e. $h^{n} \neq 1$ for all $n \neq 0$ ). The inner automorphism $\alpha(x)=h x h^{-1}, x \in X$ has no periodic points other than fixed points. The set of fixed points of $\alpha$ is the closure of $\left\{h^{n}: n \in \mathbb{Z}\right\}$ in $X$, which is a circle. Clearly $(X, \alpha)$ satisfies the descending chain condition, but $\alpha$ is not topologically transitive (or ergodic) (cf. Theorem 7.5).

(4) Let $Y=\mathbb{Z}_{/ 2}^{\mathbb{Z}}$, where $\mathbb{Z}_{/ 2}=\mathbb{Z} / 2 \mathbb{Z}$. For every $n \geq 1$, define a continuous, shift commuting, surjective homomorphism $h_{n}: Y \rightarrow Y$ by $h_{n}(y)(i)=\sum_{i \leq m \leq n+i} y(m)$. We set $f_{n}=h_{n}$ for every $n \geq 1$ and denote by $X$ the projective limit

$$
Y \stackrel{f_{1}}{\longleftarrow} Y \stackrel{f_{2}}{\longleftarrow} \ldots \stackrel{f_{n}}{\longleftarrow} Y \stackrel{f_{n+1}}{\longleftarrow} \ldots \text {. }
$$

The shift on $Y$ induces a continuous automorphism $\alpha$ of the compact, zero dimensional group $X$, and it is not difficult to verify that $\alpha$ has no periodic points 
other than the identity in $X$. The dual group $X^{\wedge}$ of $X$ is not finitely generated under $\alpha$, since $\alpha$ is not expansive. However, if $f_{n}=h_{1}$ for all $n \geq 1$, the automorphism $\alpha$ of the projective limit $X$ in (7.1) does have a dense set of periodic points as a consequence of Theorem 7.2: the homomorphism $h_{1}$ induces an automorphism $\beta$ of the projective limit $X$, and $(X, \Gamma)$ satisfies the descending chain condition, where $\Gamma \subset$ Aut $(X)$ is the abelian group generated by $\alpha$ and $\beta$. More generally, let $(k(n), n \geq 1)$ be a sequence of positive integers, put $f_{n}=h_{k(n)}, n \geq 1$, and denote by $\alpha$ the shift on the projective limit $X$ in (7.1). The proof of Theorem 7.2 can be adapted to show that the following conditions are equivalent:

(i) for every prime $p \geq 2, p$ divides $2 k(n)$ for infinitely many $n \geq 1$;

(ii) Fix $\left(\alpha^{n}\right)=\left\{1_{x}\right\}$ for every $n \geq 1$.

If $\Gamma \cong \mathbb{Z}^{d}$ with $d>1$, the topological transitivity (or ergodicity) of the $\Gamma$-action is not sufficient to ensure the density of periodic points, even if the descending chain condition is satisfied (cf. Theorem 7.5 and Corollary 7.6).

Example 7.8. Let $X=\mathrm{SU}(2)^{\mathbb{Z}}$, and let $h \in \mathrm{SU}(2)$ be an element of infinite order. Define $S \in$ Aut $(X)$ by $(S x)(n)=h x(n) h^{-1}, n \in \mathbb{Z}$, for every $x \in X$. If $T=T_{1}$ (the shift on $X)$, the abelian group $\Gamma \subset \operatorname{Aut}(X)$ generated by $(S, T\}$ does not have a dense set of periodic points (cf. Example 7.7(3)). Note that $\Gamma$ is ergodic on $X$.

Remark 7.9. Let $X$ be a compact group, $\Gamma \subset$ Aut $(X)$ a finitely generated, abelian group such that $(X, \Gamma)$ satisfies the descending chain condition, and assume that $\alpha \in$ Aut $(X)$ commutes with $\Gamma$. If $\alpha$ is ergodic the set of $\alpha$-periodic points is dense in $X$ by Corollary 7.6, but the set of $\Gamma$-periodic points need not be dense in $X$ (cf. Example 7.8). However, if $\Gamma$ satisfies the stronger condition of expansiveness, or if $X / C(X)$ is zero dimensional, then the set of $\alpha$-periodic points is dense if $\alpha$ is nonergodic, and the set of $\Gamma$-periodic points is again dense (cf. Theorem 7.2 and Corollary 7.4).

\section{The proof of Theorem 7.2}

Throughout this section $\Gamma$ will denote an infinite, finitely generated, abelian group. If $X$ is a compact group and $\alpha \in \operatorname{Aut}(X)$ we set

$$
\text { Fix }(\alpha)=\{x \in X: \alpha(x)=x\} \text {. }
$$

LEMMA 8.1. Let $X$ be a compact group, $\alpha \in$ Aut $(X)$, and let $V \subset X$ be a closed, normal, $\alpha$-invariant subgroup. Suppose that the following conditions are satisfied:

(1) for every $m \geq 1$ and $v \in V$ there exists $a w \in V$ with $v=\alpha^{m}(w) w^{-1}$;

(2) the set of $\alpha$-periodic points is dense in $V$;

(3) the set of $\alpha_{X / V}$-periodic points is dense in $X / V$;

Then the set of $\alpha$-periodic points is dense in $X$.

Proof. Let $d$ be a metric on $X$ and let $d^{\prime}$ be the metric on $X / V$ induced by $d$. We fix $x \in X$ and $\varepsilon>0$, and choose a point $u \in X / V$ such that $d^{\prime}(u, \eta(x))<\varepsilon / 2$ and $\alpha_{X / V}^{m}(u)=u$ for some $m \geq 1$, where $\eta: X \rightarrow X / V$ is the quotient map. If $y \in X$ and $w \in V$ satisfy that $\eta(y)=u$ and $\alpha^{m}(w) w^{-1}=\alpha^{m}(y) y^{-1} \in V$ then $\alpha^{m}\left(w^{-1} y\right)=w^{-1} y$ and $\eta\left(w^{-1} y\right)=u$. By assumption there exists an $\alpha$-periodic point $z \in V$ such that 
$d\left(w^{-1} y z, x\right)<\varepsilon$, and $w^{-1} y z$ is $\alpha$-periodic. Hence the set of $\alpha$-periodic points is dense in $X$.

Remark 8.2. If $V$ is a compact, abelian group and $\alpha \in$ Aut $(V)$, then $\alpha$ is ergodic if and only if $V=\left\{\alpha^{m}(w) w^{-1}: w \in V\right\}$ for every $m \geq 1$ (cf. Lemma 8.1(1)). This is a consequence of the well known fact that $\alpha$ is ergodic if and only if $\chi \cdot \alpha^{m} \neq \chi$ for every $m \geq 1$ and every nontrivial character $\chi$ of $V$ ([Ha]).

LEMMA 8.3. Let $G$ be a compact group, $H \subset G \times G$ a full subgroup, and let $Y=Y_{H} \subset G^{\mathbb{Z}}$ be the Markov subgroup given by (5.1). For every $n \in \mathbb{Z}$, define $F_{H}(n) \subset G$ by (5.3) and set $Y_{n}=Y_{H} \cap F_{H}(n)^{\mathbb{Z}}$. Then the following is true for every $n \geq 1$.

(1) The shift $T_{1}$ is ergodic on $Y_{n}$;

(2) for every $y \in Y_{n}$ and every $m \geq 1$, there exists a point $v \in Y_{n}$ such that $y=\left(T_{m} v\right) v^{-1}$;

(3) the set of shift-periodic points is dense in $Y_{n}$.

Proof. From Lemma 5.6(4) we know that there exists a Borel isomorphism $\varphi: Y_{n} \rightarrow$ $\Lambda_{1}^{\mathbb{Z}} \times \cdots \times \Lambda_{n-1}^{\mathbb{Z}}$ which carries the shift on $Y$ to the cartesian product of the shifts on $\Lambda_{1}^{\mathbb{Z}} \times \cdots \times \Lambda_{n-1}^{\mathbb{R}}$, and (1) is an immediate consequence of this.

The assertions (2) and ( 3 ) are obviously true if $n=1$, since $Y_{1} \approx \Lambda_{1}^{\mathbb{Z}}$ (cf. Proposition 5.7(1)-(2)). Using induction, we assume that (2) and (3) have been established for $n=l \geq 1$. We define $G_{k}=G / F_{H}(k)$ and $\eta^{(k)}: Y \rightarrow G_{k}^{\mathbb{Z}}, k \geq 1$, as in Proposition 5.7 and fix $y \in Y_{t+1}$. According to Proposition 5.7(2), $\boldsymbol{\eta}^{(l)}\left(Y_{l+1}\right)=\Lambda_{l+1}^{\pi}$ for some compact group $\Lambda_{l+1}$, and we conclude that there exists, for every (fixed) $m \geq 1$, a point $u \in Y_{t+1}$ such that $\boldsymbol{\eta}^{(l)}(y)=\left(S_{m} \boldsymbol{\eta}^{(l)}(u)\right) \eta^{(l)}(u)^{-1}$, where $S_{m}$ is the automorphism of $Y_{l} / Y_{l-1}$ induced by the shift $T_{m}, m \in \mathbb{Z}$. Hence $\left(T_{m} u\right)^{-1} y u \in \operatorname{ker}\left(\mathbf{y}^{(l+1)}\right)=Y_{l}$, and our induction hypothesis implies that there exists a $v \in Y_{l}$ with $\left(T_{m} u\right)^{-1} y u=$ $\left(T_{m} v\right) v^{-1}$, i.e. $y=\left(T_{m}(u v)\right)(u v)^{-1}$. This proves (2) for $n=l+1$ and hence for all $n \geq 1$. Since (2) and (3) hold for $n=l$ and $Y_{l+1} / Y_{l} \cong \eta^{(l)}\left(Y_{l+1}\right)=\Lambda_{l+1}^{\mathbb{Z}}, Y_{l+1} / Y_{l}$ has a dense set of shift-periodic points, and Lemma 8.1 establishes (3) for $n=l+1$ and hence for every $n \geq 1$.

LEMMA 8.4. Let $G$ be a compact Lie group with finite centre, and let $H \subset G \times G$ be a full subgroup. If $Y=Y_{H} \subset G^{\mathbb{Z}}$ is the Markov subgroup defined in (5.1) then the following conditions are equivalent:

(1) the set of shift-periodic points is dense in $Y$;

(2) the automorphism $\tau$ of the group $G_{k}=G / F_{H}(K)$ defined in Proposition 5.7(3) has finite order.

Proof. We use the same notation as in Proposition 5.7. There exists a $K \geq 1$ such that $F_{H}(k)=F_{H}(K)$ for all $k \geq K$, and we define $Y_{K}$ as in Proposition 5.7. If the automorphism $\tau$ induced by the shift on $G_{K}=G / F_{H}(K) \cong Y / Y_{K}$ has finite order, the set of $\tau$-periodic points is obviously dense in $Y / Y_{K}$, and the Lemmas 8.1 and 8.3 together imply that the shift-periodic points are dense in $Y_{H}$.

Now assume that $\tau$ has infinite order. Since the group of inner automorphisms of $G_{K}$ has finite index in Aut $\left(G_{K}\right)$ we can find an $M \geq 1$ such that $\tau^{M}(g)=h g h^{-1}$ for every $g \in G_{K}$, where $h \in G_{K}$ must have infinite order. We denote by $A$ the connected component of the identity in the closure of $\left\{h^{n}: n \in \mathbb{Z}\right\}$ and note that 
$A=\mathbb{R}^{d} / \mathbb{Z}^{d}$ for some $d \geq 1$. Every $g \in G_{K}$ which is periodic under $\tau$ must commute with $A$, and it follows that the closure $P$ of the set of periodic points of $\tau$ is not equal to $G_{K}$, since $A \not \subset C\left(G_{K}\right)$. The set of shift-periodic points in $Y$ is thus contained in the closed, shift-invariant set $\left(\boldsymbol{\eta}^{(K)}\right)^{-1}(P)$, and this shows that the shift-periodic points cannot be dense in $Y$.

LEMMA 8.5. Let $X$ be a compact, zero dimensional group, and let $\alpha$ be an expansive automorphism of $X$. Then the set of $\alpha$-periodic points is dense in $X$. If $\alpha$ is ergodic and $d$ a metric on $X$ there exists, for every $\varepsilon>0$, an integer $N(\varepsilon) \geq 1$ such that Fix $\left(\alpha^{n}\right)$ is $\varepsilon$-dense in $X$ for every $n \geq N(\varepsilon)$ (a set $B \subset X$ is $\varepsilon$-dense if $d(x, B)<\varepsilon$ for every $x \in X$ ).

Proof. By Theorem 5.2, Theorem 3.2, Proposition 3.12, and Proposition 5.4 we may assume that $X=Y_{H} \subset G^{\mathbb{Z}}$, where $G$ is a finite group and $H \subset G \times G$ is a full subgroup. Since $G_{K}=G / F_{H}(K)$ is finite (cf. Proposition 5.7(3)), Lemma 8.4 implies that the set of $\alpha$-periodic points is dense in $X$. If $\alpha$ is ergodic then $G_{K}=\{\mathbf{1}\}$ (cf. Proposition 5.7(5)), and there exists, for every $m \geq 1$ and $x=(x(i)) \in X$, a point $y \in$ Fix $\left(\alpha^{2 m+2 k}\right)$ with $y(i)=x(i)$ for $|i| \leq m, y(m+K)=y(-m-K)=1_{X}$. By fixing $\varepsilon>0$ and choosing $M$ sufficiently large we see that Fix $\left(\alpha^{n}\right)$ is $\varepsilon$-dense in $X$ for every $n \geq N(\varepsilon)=2 M+2 K$.

Lемма 8.6. Let $X$ be a compact, zero dimensional group, and let $\alpha \in$ Aut $(X)$ be ergodic. Then there exists, for every $x \in X$ and $m \geq 1, a v \in X$ with $x=\alpha^{m}(v) v^{-1}$.

Proof. As we have seen in Theorem 3.16, there exists a sequence $X \supset V_{1} \supset V_{2} \supset \cdots$ of closed, normal, $\alpha$-invariant subgroups of $X$ such that $\left(X(n), \alpha_{X(n)}\right)$ satisfies the descending chain condition, where $X(n)=X / V_{n}$ for every $n \geq 1$. We fix $m, n \geq 1$ and $x \in X$ for the moment. Theorem 3.2, Proposition 3.12, and Proposition 5.4 allow up to assume that $X(n)=Y_{H} \subset G^{\mathbb{Z}}$, where $G$ is a finite group and $H \subset G \times G$ a full subgroup, and that $\alpha_{X(n)}$ is the shift $T_{1}$ on $Y_{H}$. From Proposition 5.7(5) we know that $X(n)=Y_{K}$ for some $K \geq 1$, and Lemma 8.3 implies the existence of an element $v \in X$ such that $\eta_{n}(x)=\left(T_{m} \eta_{n}(v)\right) \eta_{n}(v)=\eta_{n}\left(\alpha^{m}(v) v^{-1}\right)$, where $\eta_{n}: X \rightarrow X(n)$ is the quotient map. We conclude that the set

$$
B(n)=\left\{v \in X: \eta_{n}(x)=\eta_{n}\left(\alpha^{m}(v) v^{-1}\right)\right\}
$$

is closed and nonempty for every $n \geq 1$. Since $B(1) \supset B(2) \supset \cdots \supset B(n) \supset \ldots$, the set $\bigcap_{n} B(n)=\left\{v \in X: X=\alpha^{m}(v) v^{-1}\right\}$ is nonempty. This proves our assertion.

Lemma 8.7. Let $X$ be a compact, zero dimensional group, $\Gamma \subset$ Aut $(X), V \subset X a$ $\Gamma$-invariant, closed, normal subgroup, and $\Delta \subset \Gamma$ a subgroup which is nonergodic on $V$. Then there exists a closed, $\Gamma$-invariant subgroup $W \subsetneq V$ such that $W$ is normal in $X$ and $\Delta_{V / W}$ is finite.

Proof. Let $\sigma$ be a nontrivial, continuous, irreducible, unitary representation of $V$ such that $\Delta_{\sigma}$ has finite index in $\Delta$ (cf. (2.6)). The proof of Lemma 2.2 (in particular of the implication $(2) \Rightarrow(3)$ ) yields an open, normal, $\Delta$-invariant subgroup $V^{\prime} \subsetneq V$, and Lemma 3.14 implies that $V^{\prime}$ contains a $\Delta$-invariant subgroup $W^{\prime}$ which is normal in $X$ and open in $V$. We set $F=V / W^{\prime}$ and write $\eta: V \rightarrow F$ for the quotient map. The continuous homomorphism $\eta: V \rightarrow F^{I^{\prime}}$, defined by $\eta(x)(y)=\eta(\gamma(x)), x \in V$, 
$\gamma \in \Gamma$, satisfies that $\boldsymbol{\eta}(\gamma(x))=T_{\gamma} \eta(x)$ for every $x \in X, \gamma \in \Gamma$, where $T_{\gamma}$ denotes the shift (2.3) on $F^{\Gamma}$. Furthermore,

$$
\delta_{F}\left(\eta(\gamma(x))=\eta(\gamma \delta(x))=\left(T_{\delta} \eta(x)\right)(\gamma)\right.
$$

for every $x \in V, \gamma \in \Gamma, \delta \in \Delta$. Equation (8.2) implies that the group of automorphisms $\left\{T_{\delta}: \delta \in \Delta\right\}$ is finite on $\eta(X)$. Note that $W=\operatorname{ker}(\eta)=\bigcap_{\gamma \in \Gamma} \gamma\left(W^{\prime}\right)$ is a closed, normal, $\Gamma$-invariant subgroup of $X$, and that $\Delta_{v / w} \cong\left\{T_{\delta}: \delta \in \Delta\right\}$.

Lemma 8.8. Let $X$ be a compact, zero dimensional group, $\Gamma \subset$ Aut $(X), V \subset X$ a closed, normal, $\Gamma$-invariant subgroup such that $\left(V, \Gamma_{V}\right)$ satisfies the descending chain condition, and let $\Delta \subset \Gamma$ be a subgroup such that $\Delta_{V}$ is nonergodic. Then there exists closed, normal, $\Gamma$-invariant subgroup $W \subset V \subset X$ such that $\Delta_{W}$ is ergodic and $\Delta_{V / W}$ is finite.

Proof. This follows from Lemma 8.7 and the descending chain condition: if $\Delta_{V}$ is nonergodic, there exists an open, normal, $\Delta$-invariant subgroup $W(1) \subset V=V(0)$, and we set $F(1)=V / W(1)$ and $\eta(1): X \rightarrow F^{\Gamma}$ and $V(1)=\operatorname{ker}(\eta(1))$ as in the proof of Lemma 8.7. If $\Delta_{V(1)}$ is nonergodic, there exists an open, normal, $\Delta$-invariant subgroup $W(2) \subset V(1)$, and we define $F(2), \boldsymbol{\eta}(2)$, and $V(2)=\operatorname{ker}(\boldsymbol{\eta}(2))$ as before. The descending chain condition implies that this procedure has to stop after finitely many steps: there exists an $n \geq 1$ such that $\Delta_{V(n)}$ is ergodic.

We may regard $V$ as a closed, shift-invariant subgroup of $G^{\Gamma}$, where $G$ is a finite group (cf. Theorem 3.2 and Proposition 3.12), and use Corollary 3.8 to find a finite set $D \subset \Gamma$ and normal subgroups $H(i) \subset G^{D}$ such that, for every $i=0, \ldots, n, V(i)=$ $\left\{x \in G^{\Gamma}: \pi_{D}\left(T_{\gamma} x\right) \in H(i)\right.$ for every $\left.\gamma \in \Gamma\right\}$. The open, normal subgroups $\pi_{D}^{-1}(H(i)) \subset$ $V$ contain open, normal, $\Delta$-invariant subgroups $Y(i)$ such that $V=$ $Y(0) \supset Y(1) \supset Y(2) \supset \cdots \supset Y(n)\left(\right.$ cf. Lemma 3.14), and we set $F^{\prime}=V / Y(n)$, denote by $\eta^{\prime}: V \rightarrow F^{\prime}$ the quotient map, and define $\eta^{\prime}: Y \rightarrow F^{\prime \Gamma}$ by $\eta^{\prime}(y)(\gamma)=\eta^{\prime}(\gamma(y)), \gamma \in \Gamma$, $y \in V$. As in (8.2) we see that $\Delta_{Y(n)}$ is finite, and the ergodicity of $\Delta_{V(n)}$ implies that $W=Y(n)=V(n)$. This proves that $\Delta_{V / W}$ is finite.

Lемма 8.9. Let $X$ be a compact, zero dimensional group, $\alpha \in$ Aut $(X)$, and let $V \subset X$ be a closed, normal, $\alpha$-invariant subgroup. If $\alpha_{V}$ is ergodic and $m \geq 1$ then $\eta\left(\right.$ Fix $\left.\left(\alpha^{m}\right)\right)=$ Fix $\left(\alpha_{X / V}^{m}\right)$.

Proof. Let $u \in$ Fix $\left(\alpha_{X / V}^{m}\right)$, and let $u=\eta(x)$ for some $x \in X$. Then $\alpha^{m}(x) x^{-1} \in V$, and Lemma 8.6 implies the existence of a point $v \in V$ with $\alpha^{m}(x) x^{-1}=\alpha^{m}(v) v^{-1}$. Then $v^{-1} x \in$ Fix $\left(\alpha^{m}\right)$ and $\eta\left(v^{-1} x\right)=u$. This proves our assertion.

If $X$ is a compact group, let

$$
r(X)=\infty \text { if }\left\{x \in X: x^{r} \neq 1_{X}\right\} \neq \varnothing \quad \text { for every } r \geq 1,
$$

and

$$
r(X)=\min \left\{r \geq 1: x^{r}=1_{X} \text { for every } x \in X\right\} \text { otherwise. }
$$

If the group $X$ is zero dimensional, $\Gamma \subset \operatorname{Aut}(X)$, and $(X, \Gamma)$ satisfies the descending chain condition then Theorem 3.2 and Proposition 3.12 together imply that $r(X)<\infty$.

LEMMA 8.10. Let $X$ be a compact, zero dimensional group with $r(X)<\infty$, and let $\alpha \in \operatorname{Aut}(X)$ be an automorphism with the following property: there exists an integer 
$Q \geq 1$ and closed, normal, $\alpha$-invariant subgroups

$$
X \supset V=V(0) \supset V(1) \supset \cdots \supset V(n) \supset \cdots
$$

of $X$ such that $\bigcap_{n} V(n)=\left\{\mathbf{1}_{X}\right\}$ and, for every $k \geq 1, \alpha_{V(k) / V(k+1)}$ is either ergodic, or $\alpha_{V(k) / v(k+1)}^{Q}=\mathrm{id}_{V(k) / v(k+1)}$.

Let $p \geq 2$ be an integer with $(p, \operatorname{Qr}(X))=1$ (i.e. $p$ and $\operatorname{Qr}(X)$ are relatively prime). If $\eta: X \rightarrow X / V$ is the quotient map and $\beta=\alpha_{X / V}$, then

$$
\eta\left(F i x\left(\alpha^{p}\right)\right) \supset\left\{\beta(u) u^{-1}: u \in \text { Fix }\left(\beta^{p}\right) \subset X / V\right\} .
$$

Proof. Let $\eta_{l}: X \rightarrow X / V(l)$ and $\eta_{k, l}: X / V(k) \rightarrow X / V(l)$ be the quotient maps and $\beta_{k}$ the automorphism induced by $\alpha$ on $X / V(k), 0 \leq l \leq k \leq n$. Fix $u \in$ Fix $\left(\beta^{p}\right) \subset X / V$ and choose $x(0) \in X$ with $\eta(x(0))=\eta_{0}(x(0))=u$. We construct inductively points $x(k) \in X, k \geq 1$, such that

$$
\eta_{l}(x(k))=\eta_{l}(x(l))
$$

and

$$
\beta_{k}^{Q r(X)^{k}}(u(k)) u(k)^{-1} \in \operatorname{Fix}\left(\beta_{k}^{p}\right)
$$

for all $l \geq k \geq 1$, where $u(k)=\eta_{k}(x(k))$.

Suppose that we have found $x(0), \ldots, x(j)$ with the required properties, where $j \geq 0$. If $\alpha_{V(j) / V(j+1)}$ is ergodic we apply Lemma 8.9 and choose a point $w \in$ Fix $\left(\beta_{j+1}^{p}\right) \subset X / V(j+1)$ such that $\eta_{j+1, j}(w)=\beta_{j}^{Q r(X)^{j}}(u(j)) u(j)^{-1}$. Then

$$
w=\beta_{j+1}^{Q r(X)^{j}}\left(\eta_{j+1}(x(j)) v \eta_{j+1}(x(j))^{-1}\right)
$$

for some $v \in V(j) / V(j+1)$, and Lemma 8.6 allows us to find a $v^{\prime} \in V(j) / V(j+1)$ with

$$
v=\beta_{j+1}^{\operatorname{Qr}(X)^{i}}\left(v^{\prime}\right) v^{\prime-1}=\left(\alpha \underset{v(j) / V(j+1)}{\operatorname{Qr}(X)^{j}}\right)\left(v^{\prime}\right) v^{-1} \text {. }
$$

We choose $x(j+1) \in X$ such that $u(j+1)=\eta_{j+1}(x(j+1))=\eta_{j+1}(x(j)) v^{\prime}$ and note that $\eta_{k}(x(j+1))=\eta_{k}(x(j))$ for $k \leq j$ and

$$
\beta_{j+1}^{Q r(X)^{i}}(u(j+1)) u(j+1)^{-1}=w \in \operatorname{Fix}\left(\beta_{j+1}^{p}\right)
$$

and hence

$$
\beta_{j+1}^{Q r(X)^{j+1}}(u(j+1)) u(j+1)^{-1} \in \operatorname{Fix}\left(\beta_{j+1}^{p}\right)
$$

This construction yields the required point $x(j+1)$ under the assumption that $\alpha_{v(j) / v(j+1)}$ is ergodic. If $\alpha_{v(j) / v(j+1)}$ is nonergodic then $\alpha_{V(j) / v(j+1)}^{Q}=\mathrm{id}_{v(j) / v(j+1)}$. We put $u^{\prime}=\eta_{j+1}(x(j))$ and observe that

$$
\beta_{j+1}^{p}\left(\beta_{j+1}^{Q r(X)^{\prime}}\left(u^{\prime}\right) u^{\prime-1}\right)=\beta_{j+1}^{Q r(X)^{i}}\left(u^{\prime}\right) v u^{\prime-1}
$$

for some $v \in V(j) / V(j+1)$. Hence

$$
\beta_{j+1}^{Q r(X)^{\prime}}\left(u^{\prime-1} \beta_{j+1}^{p}\left(u^{\prime}\right)\right)=v u^{\prime-1} \beta_{j+1}^{p}\left(u^{\prime}\right),
$$

and

$$
\beta_{j+1}^{Q i r(X)^{\prime}}\left(u^{\prime-1} \beta_{j+1}^{p}\left(u^{\prime}\right)\right)=v^{i} u^{\prime-1} \beta_{j+1}^{p}\left(u^{\prime}\right)
$$

for every $i \geq 1$, since $\beta_{j+1}^{Q r(X)^{\prime}}(v)=\beta_{j+1}^{Q}(v)=\alpha_{v(j) / v(j+1)}^{Q}(v)=v$. By setting $i=r(X)$ we see that

$$
\beta_{j+1}^{\operatorname{Qr}(X)^{\prime+1}}\left(u^{\prime-1} \beta_{j+1}^{p}\left(u^{\prime}\right)\right)=u^{\prime-1} \beta_{j+1}^{p}\left(u^{\prime}\right)
$$

or, equivalently, that

$$
\beta_{j+1}^{p}\left(\beta_{j+1}^{Q r(X)^{j+1}}\left(u^{\prime}\right) u^{\prime-1}\right)=\beta_{j+1}^{Q r(X)^{j+1}}\left(u^{\prime}\right) u^{\prime-1}
$$


It follows that the point $x(j+1)=x(j)$ satisfies (8.5) and (8.6).

This induction process yields, for every $n \geq 1$, a point $x(n) \in X$ satisfying (8.5) and (8.6). We fix $n$ for the moment and note that, since $(p, \operatorname{Qr}(X))=1$, there exist integers $a, b$ such that $a \operatorname{Qr}(X)^{n}+b p=1$. Then

and

$$
\beta_{n}^{a Q r(X)^{n}}(u(n)) u(n)^{-1}=\eta_{n}\left(\alpha^{a Q r(X)^{n}}(x(n)) x(n)^{-1}\right) \in \operatorname{Fix}\left(\beta_{n}^{p}\right)
$$

$$
\eta\left(\alpha^{a Q r(X)^{\prime \prime}}(x) x^{-1}\right)=\beta^{a Q r(X)^{\prime \prime}+b p}(u) u^{-1}=\beta(u) u^{-1} .
$$

We have proved that

$$
F(n)=\left\{x \in X: \eta_{n}(x) \in \text { Fix }\left(\beta_{n}^{p}\right) \text { and } \eta(x)=\beta(u) u^{-1}\right\} \neq \varnothing
$$

for every $n \geq 1$. Clearly $F(1) \supset \cdots \supset F(n) \supset \cdots, F=\bigcap_{n} F(n) \neq \varnothing$, and $F \subset$ Fix $\left(\alpha^{p}\right) \cap \eta^{-1}\left(\left\{\beta(u) u^{-1}\right\}\right)$. This proves (8.4), since $u \in \operatorname{Fix}\left(\beta^{p}\right)$ was arbitrary.

LеммA 8.11. Let $X$ be a compact, zero dimensional group and let $\Gamma \subset$ Aut $(X)$ be expansive. Then the set of $\Gamma$-periodic points is dense in $X$.

Proof. (1) Initial reduction. According to Theorem 3.2, Proposition 3.12, and Theorem 5.2 we may regard $X$ as a closed, shift-invariant subgroup of $G^{\Gamma}$, where $G$ is a finite group, and the last step in the proof of Theorem 3.2 allows us to assume that $\Gamma=\mathbb{Z}^{d}$ for some $d \geq 1$. If $d=1$, and if $d$ is a metric on $X \subset G^{\mathbb{Z}}$, Lemma 8.5 shows that the set of shift-periodic points is dense in $X$, and that, for every $\varepsilon>0$, Fix $\left(T_{n}\right)$ is $\varepsilon$-dense in $X$ for all sufficiently large $n \geq 1$.

We use induction and assume that, for some $d \geq 1$, every finite group $G$, and every closed, shift-invariant subgroup $X \subset G^{\mathbb{Z}^{d}}$, the following conditions are satisfied.

The set of shift-periodic points is dense in $X$;

if $d$ is a metric on $X$, and if $T_{m}$ is ergodic on $X$ for some $m \in \mathbb{Z}^{d}$, there exists an integer $L(m)$ such that Fix $\left(T_{k m}\right)$ is $\varepsilon$-dense in $X$ for all sufficiently large $k \geq 1$ with $(k, L(\boldsymbol{m}))=1$ (i.e. $k$ and $L(\boldsymbol{m})$ are relatively prime).

Let $G$ be a finite group, $X \subset G^{\mathbb{Z}^{d+1}}$ a closed, shift-invariant subgroup, $d$ a metric on $X$, and let $\boldsymbol{m}=\left(m_{1}, \ldots, m_{d+1}\right) \in \mathbb{Z}^{d+1}$. We denote by $a=\left(m_{1}, \ldots, m_{d+1}\right)$ the highest common factor of $\left\{m_{1}, \ldots, m_{d+1}\right\}$ and set $\boldsymbol{m}^{\prime}=\left(m_{1}^{\prime}, \ldots, m_{d+1}^{\prime}\right)=$ $\left(m_{1} / a, \ldots, m_{d+1} / a\right)$. Suppose that we can prove the following:

(1) the set of $T_{m^{\prime}}$-periodic points is dense in $X$;

(2) if $T_{\boldsymbol{m}^{\prime}}$ is ergodic on $X$ there exists an integer $L\left(\boldsymbol{m}^{\prime}\right) \geq 1$ such that, for every $\varepsilon>0$, Fix $\left(T_{k m^{\prime}}\right)$ is $\varepsilon$-dense in $X$ for all $k \geq 1$ with $\left(k, L\left(m^{\prime}\right)\right)=1$.

Then $T_{\boldsymbol{m}}$ satisfies (8.10) with $\boldsymbol{m}$ replacing $\boldsymbol{m}^{\prime}$ and with $L(\boldsymbol{m})=a L\left(\boldsymbol{m}^{\prime}\right)$. Let $\Delta^{\prime} \subset \mathbb{Z}^{d+1}$ be a subgroup such that $\Delta^{\prime} \cong \mathbb{Z}^{d}$ and $\left\{n+k m^{\prime}: n \in \Delta^{\prime}\right.$ and $\left.k \in \mathbb{Z}\right\}=\mathbb{Z}^{d+1}$. For every $n \geq 1$, the set $Y(n)=$ Fix $\left(T_{n m^{\prime}}\right)$ is a closed, shift-invariant subgroup of $X$, and $\left(Y(n), T_{\Delta^{\prime}}\right)$ satisfies the descending chain condition. Our induction hypothesis (8.7) implies that the set of $T_{\Delta}$-periodic points is dense in $Y(n)$ for every $n \geq 1$, and we conclude that $X$ satisfies (8.7). Hence (8.7) and (8.8) are satisfied with $d+1$ replacing $d$, and the lemma is proved.

There exists a matrix $A \in G L(d+1, \mathbb{Z})$ with $A m^{\prime}=e=(0, \ldots, 0,1) \in \mathbb{Z}^{d+1}$, and the automorphism $\xi \in$ Aut $\left(G^{\mathbb{Z}^{d+1}}\right)$ given by $(\xi y)(\boldsymbol{k})=y\left(A^{-1} k\right), k \in \mathbb{Z}^{d+1}, y \in G^{\mathbf{Z}^{d+1}}$, 
satisfies that $\xi T_{e} \xi^{-1}=T_{m}$. Furthermore $\xi$ sends the set of shift-periodic points in $X$ to the set of shift-periodic points in $\xi(X)$. If we can prove (8.9) and (8.10) for $\xi(X)$ and $T_{e}$ then the corresponding statements hold for $X$ and $T_{m}$, with $L\left(m^{\prime}\right)=L(e)$. This allows us to assume that $\boldsymbol{m}^{\prime}=\boldsymbol{e}$ in (8.9) and (8.10).

(2) The proof of (8.9) and (8.10) in the ergodic case. Assume that $T_{e}$ is ergodic. According to Corollary 3.11 we may also assume that

$$
X=\left\{x \in G^{\mathbb{Z}^{\prime \prime+1}}: \pi_{I(d+1)}\left(T_{n} x\right) \in H \text { for every } n \in \mathbb{Z}^{d+1}\right\},
$$

where $I(d+1)=\{0,1\}^{d+1}$ and $H=\pi_{I(d+1)}(X) \subset G^{I(d+1)}$. Put

$$
\Delta=\left\{\boldsymbol{n}=\left(n_{1}, \ldots, n_{d+1}\right) \in \mathbb{Z}^{d+1}: n_{1}=0\right\}
$$

and set, for every $k \geq 0, \Delta(k)=\left\{n=\left(n_{1}, \ldots, n_{d+1}\right) \in \mathbb{Z}^{d+1}:\left|n_{1}\right| \leq k\right\}$ and $Y(k)=$ $\pi_{\Delta(k)}(X)(\mathrm{cf} .(2.2))$.

Let $F^{\prime} \subset F \subset \mathbb{Z}^{d+1}$. In order to keep notation simple we shall not distinguish notationally between the projection $\pi_{F^{\prime}}: X \subset G^{\mathbb{Z}^{\prime \prime+1}} \rightarrow G^{F^{\prime}}$ and the map from $\pi_{F}(X)$ to $G^{F^{\prime}}$ induced by $\pi_{F^{\prime}}$, and we write $T_{د}=\left\{T_{n}: n \in \Delta\right\}$ for the shift-action of $\Delta$ on $\boldsymbol{X}$ as well as on any $T_{\Delta}$-invariant subgroup or quotient group of $\boldsymbol{X}$. For every $k \geq 0$, put $W(k)=\operatorname{ker}\left(\pi_{\Delta(k)}\right)$ and $Y(k)=\pi_{\Delta(k)}(X)$, and observe that $\left(Y(k), T_{\Delta}\right)$ satisfies the descending chain condition. According to (8.11), the pairs $\left(W(k) / W(k+1), T_{e}\right)$, $k \geq 0$, are all conjugate (cf. (2.1)). Lemma 8.8, applied to $W(0) / W(1)$, allows us to choose a closed, normal, $T_{\perp}$-invariant subgroup $W(1) \subset V(1) \subset V(0)=W(0) \subset X$ such that $T_{e}$ is ergodic on $V(1) / W(1)$ and has finite order $Q$ on $V(0) / V(1)$. From the inclusions $W(0) \supset W(1) \supset \cdots \supset W(k) \supset \cdots$ we obtain a sequence

$$
W(0)=V(0) \supset V(1) \supset W(1)=V(2) \supset \cdots \supset V(2 n)=W(n) \supset \cdots
$$

of closed, normal, $T_{د}$-invariant subgroups of $X$ such that $\left(V(i) / V(i+1), T_{e}\right)$ is conjugate to $\left(V(i+2 k) / V(i+1+2 k), T_{e}\right)$ for all $i=0,1$ and $k \geq 1$. We can thus apply Lemma 8.10 and conclude that

$$
\pi_{\Delta(k)}\left(\left\{x \in X: T_{p e} x=x\right\}\right) \supset\left\{T_{e}(u) u^{-1}: u \in Y(K) \text { and } T_{p e} u=u\right\}
$$

for all $K, l \geq 1$, and for all $p \geq 2$ with $(p, Q r(X))=1$.

Let $\varepsilon>0$. There exists an integer $K \geq 1$ such that $d\left(x, x^{\prime}\right)<\varepsilon / 2$ for all $x, x^{\prime} \in X$ with $\pi_{\Delta(K)}(x)=\pi_{\Delta(K)}\left(x^{\prime}\right)$. Since $T_{e}$ is ergodic on $Y(K)$ the map $\partial_{e}: Y(K) \rightarrow Y(K)$ defined by $\partial_{e}(y)=\left(T_{e} y\right) y^{-1}, y \in Y(K)$, is continuous and surjective (Lemma 8.6). Hence there exists an $\varepsilon^{\prime}>0$ such that $\partial_{e}(B)$ is $\varepsilon / 2$-dense in $X$ for every $\varepsilon^{\prime}$-dense set $B \subset Y(K)$. The pair ( $Y(K), T_{د}$ ) satisfies the descending chain condition, and the induction hypothesis (8.8) implies that there exists an integer $L \geq 1$ such that $\left\{y \in Y(K): T_{n e} y=y\right\}$ is $\varepsilon^{\prime}$-dense in $Y(K)$ for all sufficiently large $n \geq 1$ with $(n, L)=$ 1. Then $\left\{T_{e}(u) u^{-1}: u \in Y(K)\right.$ and $\left.T_{n e} u=u\right\}$ is $\varepsilon / 2$-dense in $Y(K)$, and from (8.13) and the choice of $K$ it is clear that Fix $\left(T_{n e}\right)$ is $\varepsilon$-dense in $X$ for all sufficiently large $n \geq 1$ with $(n, L Q r(X))=1$. By setting $L\left(T_{e}\right)=L Q r(X)$ we have proved (8.9) and (8.10), and hence (8.7), if $T_{e}$ is ergodic.

(3) The proof of (8.9) in the nonergodic case. If $T_{e}$ is nonergodic on $X$ we apply Lemma 8.8 and find a closed, normal, shift-invariant subgroup $W \subset X$ such that $T_{e}$ has finite order on $X / W$ and is ergodic on $W$. From part (2) of this proof we know 
that the set of $T_{e}$-periodic points is dense in $W$, and the Lemmas 8.1 and 8.6 show that the set of $T_{e}$-periodic points is dense in $X$. This proves $(8.9)$ if $T_{e}$ is nonergodic.

The proof of Lemma 8.11 is complete.

Proof of Theorem 7.2. Corollary 4.8 and Lemma 8.11 .

\section{The ascending chain condition and the proof of Theorem 7.3}

Although Theorem 7.3 is a special case of Theorem 3.2 in [La], where $\Gamma \subset \operatorname{Aut}(X)$ is assumed to be an arbitrary semigroup, we present here an alternative proof based on the fact that, if $G$ is a compact, connected Lie group with trivial centre, then the closed, normal, shift-invariant subgroups of $G^{\Gamma}$ satisfy both the ascending and descending chain conditions.

LEMMA 9.1. Let $X$ be a compact, connected group with trivial centre, $\Gamma \subset$ Aut $(X)$, and assume that $(X, \Gamma)$ satisfies the descending chain condition. Then there exists a compact, connected Lie group $G$ with trivial centre and a continuous, injective homomorphism $\varphi: X \rightarrow G^{\Gamma}$ such that $\varphi(X)$ is a full, shift-invariant subgroup of $G^{r}$, and $\varphi(\gamma(x))=T_{\gamma} \varphi(x)$ for every $x \in X, \gamma \in \Gamma$.

Proof. According to Theorem 3.2 we may regard $X$ as a full, shift-invariant subgroup of $H^{\mathbf{r}}$, where $H$ is a compact (and necessarily connected) Lie group. We denote by $\eta: H \rightarrow H / C(H)=G$ the quotient map and define a continuous homomorphism $\boldsymbol{\eta}: H^{\Gamma} \rightarrow G^{\Gamma^{*}}$ by $\boldsymbol{\eta}(x)(\gamma)=\eta(x(\gamma)), \gamma \in \Gamma, x \in H^{\Gamma^{*}}$. The restriction $\varphi$ of $\boldsymbol{\eta}$ to $X$ is injective, since $\operatorname{ker}(\eta) \cap X=C(X)=\left\{1_{X}\right\}$, and has the required properties.

LEMMA 9.2. Let $G$ be a compact Lie group with finite centre, $G^{\prime}=G / C(G), \eta: G \rightarrow G^{\prime}$ the quotient map, and define $\boldsymbol{\eta}: G^{\Gamma} \rightarrow G^{I^{\prime}}$ by setting $\boldsymbol{\eta}(x)(\gamma)=\eta(x(\gamma)), \gamma \in \Gamma, x \in X$. If $X \subset G^{\Gamma}$ is a closed, shift-invariant, expansive subgroup then $\eta(X)$ is a closed, shift-invariant, expansive subgroup of $G^{\prime \mathrm{I}^{\mathrm{T}}}$.

Proof. Let $d$ be a metric on $G$ and denote by $d^{\prime}$ the induced metric on $G^{\prime}$. We choose and fix $\delta>0$ such that $d\left(g, g^{\prime}\right)>2 \delta$ whenever $g, g^{\prime} \in C(G)$ and $g \neq g^{\prime}$, and set

$$
B(\varepsilon)=\{x \in X: d(x(\gamma), C(G))<\varepsilon \text { for every } \gamma \in \Gamma\}
$$

for every $\varepsilon>0$. Now assume that $2 \varepsilon<\delta$. For every $x \in B(\varepsilon)$ there exists a unique element $z_{x} \in C(G)^{\top}$ such that $d\left(x(\gamma), z_{x}(\gamma)\right)<\varepsilon$ for every $\gamma \in \Gamma$. If $\varepsilon$ is small (e.g. $\varepsilon<\delta / 4)$ then $z_{x x^{\prime}}=z_{x} z_{x^{\prime}}$ for all $x, x^{\prime} \in B(\varepsilon)$. We denote the order of $C(G)$ by $M$ and observe that, for $\varepsilon<\delta / 2 M, z_{x}{ }^{M}=\left(z_{x}\right)^{M}=1_{G^{\prime}}$ for every $x \in Z(\varepsilon)$. Since $X$ is expansive we conclude that $\boldsymbol{x}^{M}=\mathbf{1}_{X}$ for every $x \in B(\varepsilon)$ with $\varepsilon<\delta / 2 M$. By decreasing $\varepsilon$, if necessary, we may assume that

$$
\left\{g \in G: g^{M}=\mathbf{1}_{G} \text { and } d\left(g, \mathbf{1}_{G}\right)<\varepsilon\right\}=\left\{\mathbf{1}_{G}\right\}
$$

and hence that $B(\varepsilon) \subset C(G)^{\mathrm{F}}$. This proves that

$$
\boldsymbol{\eta}(B(\varepsilon))=\left\{x^{\prime} \in \boldsymbol{\eta}(X): d^{\prime}\left(x^{\prime}(\gamma), \mathbf{1}_{G^{\prime}}\right)<\varepsilon \text { for all } \gamma \in \Gamma\right\}=\left\{\mathbf{1}_{\boldsymbol{\eta}(X)}\right\}
$$

for all sufficiently small $\varepsilon>0$, i.e. that $\boldsymbol{\eta}(X)$ is expansive.

LEMMA 9.3. Let $G$ be a compact, connected Lie group with trivial centre and let $X \subset G^{\mathbf{r}}$ 
be a full, connected, shift-invariant subgroup. For every closed, normal, shift invariant subgroup $V \subset X$ we put

$$
V^{*}=\{x \in X: x v=v x \text { for all } v \in V\}
$$

Then $V^{*} \cap V=\left\{\mathbf{1}_{X}\right\}, V \cdot V^{*}=X$, and $V^{* *}=\left(V^{*}\right)^{*}=V$ for every closed, normal, shift-invariant subgroup $V \subset X$. Furthermore every closed, normal, shift-invariant subgroup $V \subset X$ is connected.

Proof. First assume that $V=X$. Since $C(X) \subset C(G)^{1}=\left\{\mathbf{1}_{G^{\prime}}\right\}$ we have that $X^{*}=\left\{\mathbf{1}_{X}\right\}$ and $X^{* *}=X$. If $V \subsetneq X$ is a closed, normal, shift-invariant subgroup of $X$ we apply Corollary 3.8 and choose a finite set $D \subset \Gamma$ such that

$$
X=\left\{x \in G^{\Gamma}: \pi_{D}\left(T_{\gamma} x\right) \in \pi_{D}(X) \text { for every } \gamma \in \Gamma\right\}
$$

and

$$
V=\left\{x \in G^{\mathrm{r}}: \pi_{D}\left(T_{\gamma} x\right) \in \pi_{D}(V) \text { for every } \gamma \in \Gamma\right\} .
$$

Let $(E(n), n \geq 1)$ be an increasing sequence of finite sets such that $D \subset E(n)$ for every $n \geq 1$ and $\bigcup_{n} E(n)=\Gamma$. We fix $n \geq 1$ for the moment and note that, since $G$ has trivial centre and $X \subset G^{1}$ is full, $\pi_{E(n)}(X) \subset G^{E(n)}$ is a compact, connected Lie group with trivial centre. Furthermore $\pi_{E(n)}(V)$ is a closed, normal subgroup of $\pi_{E(n)}(X)$. Clearly

$$
H_{n}=\left\{h \in \pi_{E(n)}(X): h u=u h \text { for every } u \in \pi_{E(n)}(V)\right\}
$$

is a closed, connected, normal, subgroup of $\pi_{E(n)}(X), \pi_{E(n)}(V) \cap H_{n}=\{\mathbf{1}\}$, and $\pi_{E(n)}(X)=\pi_{E(n)}(V) \cdot H_{n}$. Since the Lie groups $\pi_{E(k)}(X), k \geq 1$, all satisfy the descending chain condition on closed (normal) subgroups there exists, for every $k \geq 1$, an integer $m(k) \geq k$ with $\pi_{E(k)}\left(H_{n}\right)=\pi_{E(k)}\left(H_{m(k)}\right)$ for all $n \geq m(k)$. Put

$$
C_{n}=\left\{x \in X: \pi_{E(n)}(x) \in H_{n}\right\}=\left\{x \in X: \pi_{E(n)}(x v)=\pi_{E(n)}(v x) \text { for every } v \in V\right\} \text {. }
$$

The sequence $\left(C_{n}, n \geq 1\right)$ of closed, normal subgroups of $X$ decreases, $V^{*}=\bigcap_{n} C_{n}$, $\pi_{E(k)}\left(V^{*}\right)=\pi_{E(k)}\left(H_{m(k)}\right)$ for every $k \geq 1$, and $V^{*}$ is a closed, normal, shift-invariant subgroup of $X$.

From the definition of $V^{*}$ it is clear that $V^{* *} \supset V$. If $V \subsetneq V^{* *}$ then $\pi_{E(k)}(V) \subsetneq$ $\pi_{E(k)}\left(V^{* *}\right) \subset \pi_{E(k)}\left(\pi_{E(m(k))}(V)\right)=\pi_{E(k)}(V)$ for all sufficiently large $k \geq 1$, which is absurd. Hence $V^{* *}=V$, as claimed. Finally we note that, for every $k \geq 1$,

$$
\begin{aligned}
\pi_{E(k)}\left(V \cdot V^{*}\right) & =\pi_{E(k)}(V) \cdot \pi_{E(k)}\left(V^{*}\right)=\pi_{E(k)}\left(\pi_{E(m(k))}(V) \cdot H_{m(k)}\right) \\
& =\pi_{E(k)}\left(\pi_{E(m(k))}(X)\right)=\pi_{E(k)}(X) .
\end{aligned}
$$

We conclude that, for every $x \in X, P_{k}(x)=\left\{(v, u) \in V \times V^{*}: \pi_{E(k)}(v u)=\pi_{E(k)}(x)\right\} \neq$ $\varnothing$ and $P_{k}(x) \supset P_{k+1}(x)$ for every $k \geq 1$. Hence $\bigcap_{k} P_{k}(x)=P(x) \neq \varnothing$, and $x=v u$ for every $(v, u) \in P(x) \subset V \times V^{*}$. This proves that $V \cdot V^{*}=X$ and $V \cap V^{*}=\left\{1_{X}\right\}$. In particular $V \cong X / V^{*}$ and hence connected.

LEMмA 9.4. Let $G$ be a compact, connected Lie group with trivial centre, and let $X \subset G^{\mathrm{I}}$ be a full, connected, shift-invariant subgroup of $X$. Then $X$ satisfies both the ascending and the descending chain conditions on closed, normal, shift-invariant subgroups (the ascending chain condition is defined in analogy to the descending chain condition for increasing sequences of shift-invariant subgroups). 
Proof. From Theorem 3.2 we know that $X$ satisfies the descending chain condition on closed, shift-invariant subgroups. If $\left(V_{n}, n \geq 1\right)$ is an increasing sequence of closed, normal, shift-invariant subgroups of $X$ we define $V_{n}^{*}, n \geq 1$, by (9.1) and obtain a decreasing sequence $\left(V_{n}^{*}, n \geq 1\right)$ of closed, normal, shift-invariant subgroups of $X$. The descending chain condition implies that there exists a $k \geq 1$ with $V_{n}^{*}=V_{k}^{*}$ for every $n \geq k$, and hence $V_{n}=V_{n}^{* *}=V_{k}^{* *}=V_{k}$ for every $n \geq k$. This proves the ascending chain condition.

The Lemmas 9.1 and 9.4 have the following interesting consequence.

Proposition 9.5. Let $X$ be a compact, connected group with trivial centre, $\Gamma \subset$ Aut $(X)$, and assume that $(X, \Gamma)$ satisfies the descending chain condition. Then $X$ satisfies both the ascending and the descending chain conditions on closed, normal, $\Gamma$-invariant subgroups.

Let $X$ be a compact, connected group with trivial centre and let $\alpha \in \operatorname{Aut}(X)$ be an expansive automorphism. Then Theorem 5.3 implies that $X$ is trivial (i.e. $X=\{1\}$ ). We proceed by induction and assume that $d \geq 2$, and that we have proved the following: if $X$ is a compact, connected group with trivial centre and $\Gamma \subset$ Aut $(X)$ a group such that $\Gamma \cong \mathbb{Z}^{d-1}$ and $\Gamma$ acts expansively on $X$ then $X=\{1\}$.

LемMA 9.6. Let $G \neq\{1\}$ be a compact, connected Lie group with trivial centre and let $X \subset G^{\mathbb{Z}^{\prime \prime}}$ be a connected, expansive, full, shift-invariant subgroup. Then $T_{n}$ is ergodic on $X$ for every $\mathbf{0} \neq \boldsymbol{n} \in \mathbb{Z}^{d}$.

Proof. We fix a primitive element $\boldsymbol{n}=\left(n_{1}, \ldots, n_{d}\right) \in \mathbb{Z}^{d}$ (i.e. $\boldsymbol{n} \neq 0$, and the highest common factor of $\left\{n_{1}, \ldots, n_{d}\right\}$ is 1$)$, and choose a subgroup $\Delta \subset \mathbb{Z}^{d}$ with $\Delta \cong \mathbb{Z}^{d-1}$ and $\{\boldsymbol{m}+k \boldsymbol{n}: \boldsymbol{m} \in \Delta, k \in \mathbb{Z}\}=\mathbb{Z}^{d}$. If $T_{\boldsymbol{n}}$ is nonergodic on $X$ we apply Lemma 2.2 and find a closed, normal, $T_{n}$-invariant subgroup $V \subsetneq X$ and a metric $d^{\prime}$ on $X / V$ which is invariant under the automorphism $S_{n}$ induced by $T_{n}$ on $W=X / V$. We write $\eta: X \rightarrow W$ for the quotient map and define a continuous homomorphism $\eta: X \rightarrow W^{\mathbb{Z}^{d}}$ by setting $\boldsymbol{\eta}(x)(\boldsymbol{m})=\boldsymbol{\eta}(x(\boldsymbol{m})), \boldsymbol{m} \in \mathbb{Z}^{d}, x \in X$. Since

$$
\left(T_{n} \eta(x)\right)(\boldsymbol{m})=\boldsymbol{\eta}(x)(\boldsymbol{m}+\boldsymbol{n})=\eta\left(T_{n} T_{m} x\right)=S_{n} \eta\left(T_{m} x\right)
$$

for every $m \in \mathbb{Z}^{d}, x \in X$, it is clear that there exists a metric on $\eta(X) \subset W^{\mathbb{Z}^{\prime \prime}}$ which is invariant under $T_{n}$ (we are using the same symbol $T_{m}$ to denote the shift on $X \subset G^{\mathbb{Z} d}$ and on $\left.\eta(X) \subset W^{\mathbb{Z}^{d}}\right)$.

Now consider the closed, normal, shift-invariant subgroup $Y=\operatorname{ker}(\boldsymbol{\eta}) \subset X$ and define the closed, connected, normal, shift-invariant subgroup $Y^{*} \subset X$ by (9.1). Lemma 9.3 implies that $X=Y \cdot Y^{*}$ and $Y \cap Y^{*}=\left\{\mathbf{1}_{X}\right\}$. In particular the group $Y^{*}$ has trivial centre, and there exists a continuous group isomorphism $\psi: \eta(X) \cong$ $X / Y \rightarrow Y^{*}$ such that $\psi \cdot T_{m}=T_{m} \cdot \psi$ for every $m \in \mathbb{Z}^{d}$.

By combining this with the first part of this proof we see that there exists a metric on the subgroup $Y^{*} \subset X$ which is invariant under $T_{n}$, and conclude that $T_{د}=$ $\left\{T_{\boldsymbol{m}}: \boldsymbol{m} \in \Delta\right\}$ acts expansively on $Y^{*}$. Since $Y^{*}$ is connected and has trivial centre and $\Delta \cong \mathbb{Z}^{d-1}$, our induction hypothesis implies that $Y^{*}=\{1\}$, i.e. that $Y=X$ (cf. Lemma 9.3). This violates our earlier assertion that $V \subsetneq X$, and the resulting contra- 
diction implies that $T_{n}$ is ergodic - and hence mixing - on $X$ for every primitive $\boldsymbol{n} \in \mathbb{Z}^{d}$ (cf. Theorem 2.4). It follows that $T_{\boldsymbol{m}}$ is ergodic on $X$ for every $\mathbf{0} \neq \boldsymbol{m} \in \mathbb{Z}^{d}$.

LEMMA 9.7. Let $G$ be a compact, connected Lie group with trivial centre and let $X$ $\subset G^{\mathbb{Z}^{d}}$ be a connected, expansive, full, shift-invariant subgroup. Then $G=\{1\}$.

Proof. Without loss in generality we assume that

$$
X=\left\{x \in G^{\mathbb{Z}^{d}}: \pi_{I(d)}\left(T_{n} x\right) \in \pi_{I(d)}(X) \text { for every } n \in \mathbb{Z}^{d}\right\},
$$

where $I(d)=\{0,1\}^{d} \subset \mathbb{Z}^{d}$ (cf. Corollary 3.11). Put $e=(1,0, \ldots, 0) \in \mathbb{Z}^{d}, \Delta=$ $\left\{\boldsymbol{n}=\left(n_{1}, \ldots, n_{d}\right) \in \mathbb{Z}^{d}: n_{1}=0\right\}$, and $W=\pi_{\Delta}(X) \subset G^{\mathbb{Z}^{l-1}}$. Then $W$ is a full, connected, shift-invariant subgroup of $G^{\mathbb{Z}^{\prime-1}}$, and we define a continuous, injective homomorphism $\eta: X \rightarrow W^{\mathbb{Z}}$ by setting $\eta(x)(n)=\pi_{\Delta}\left(T_{n e} x\right), n \in \mathbb{Z}, x \in X$. The group $\eta(X) \subset W^{\mathbb{Z}}$ is full, connected, and shift-invariant, and (9.2) implies that $\eta(X)=Y_{H}$, where $H=\pi_{\{0,1\}}(\eta(X))$ and $Y_{H}$ is the Markov subgroup of $W^{\mathbb{Z}}$ given by (5.1). We define the closed, normal, shift-invariant subgroups $F_{H}(n) \subset W, n \in \mathbb{Z}$, as in (5.4) (with $W$ replacing $G$ ) and conclude from (5.5) and Lemma 9.3 that there exists an integer $K \geq 1$ such that $F_{H}(k)=F_{H}(K)$ for all $k \geq K$.

Next we prove that $F_{H}(K)=W$. Indeed, if $D \subset \Delta$ is a finite set, then $\pi_{D}(W) \subset G^{D}$ is a full, connected subgroup and hence a compact, connected Lie group with trivial centre. We define a continuous homomorphism $\psi: Y_{H} \rightarrow\left(\pi_{D}(W)\right)^{\mathbb{Z}}$ by $\psi(x)(n)=$ $\pi_{D}(x(n)), n \in \mathbb{Z}, x \in Y_{H} \subset W^{\mathbb{Z}}$, and note that $\psi \cdot T_{1}=S_{1} \cdot \psi$, where $T_{1}$ and $S_{1}$ are the shifts on $W^{\mathbb{Z}}$ and $\left(\pi_{D}(W)\right)^{\mathbb{Z}}$, respectively. Since $T_{e}$ is ergodic on $Y_{H}$ by Lemma 9.6, $S_{1}$ is ergodic on $\psi\left(Y_{H}\right)$. For every $n \geq 1$ we put $F^{\prime}(n)=\pi_{D}\left(F_{H}(n)\right)$, and the ergodicity of $S_{1}$ on $\psi\left(Y_{H}\right)$ implies that there exists an $L \geq 1$ such that $\pi_{D}(W)=$ $F^{\prime}(L)=\pi_{D}\left(F_{H}(L)\right)=\pi_{D}\left(F_{H}(K)\right)$ (cf. Proposition 5.7(5)). We have thus shown that $\pi_{D}\left(F_{H}(K)\right)=\pi_{D}(W)$ for every finite set $D \subset \Delta$ and hence that $F_{H}(K)=W$, as claimed.

From the definition of $Y_{H}$ and $F_{H}(n), n \in \mathbb{Z}$, it is clear that there exists, for every $w \in W$, a point $y \in Y_{H}$ with $y(0)=y(2 K)=1_{W}, y(K)=w$, and $T_{2 K} y=y$. In other words, the set $U=\left\{y \in Y_{H}: T_{2 K} y=y\right\}$ is a full subgroup of $Y_{H}$. For every $n \geq 1$, the group $F_{H}(n)$ is connected by Lemma 9.3, and this is easily seen to imply the connectedness of $U$. Hence $V=\left\{x \in X: T_{2 K e} x=x\right\} \subset G^{\mathbb{Z}^{d}}$ is a full, connected, shiftinvariant subgroup of $G^{\mathbb{Z}^{\prime \prime}}$ and has trivial centre. The group $T_{\Delta}=\left\{T_{n}: n \in \Delta\right\} \cong \mathbb{Z}^{d-1}$ acts expansively on $V$, and our induction hypothesis implies that $V=\{\mathbf{1}\}$. Hence $X=G=\{\mathbf{1}\}$, since $V$ was a full, connected subgroup of $G^{\mathbb{Z}^{\prime \prime}}$.

In view of the induction process described in the paragraph preceding Lemma 9.6, the Lemmas 9.1 and 9.7 imply the following result.

LEMMA 9.8. Let $d \geq 1$, and let $X$ be a compact, connected group with trivial centre and $\Gamma \subset$ Aut $(X)$ a group such that $\Gamma \cong \mathbb{Z}^{d}$ and $\Gamma$ acts expansively on $X$. Then $X=\{1\}$.

Proof of Theorem 7.3. Let $X$ be a compact, connected group and assume that $\Gamma \subset$ Aut $(X)$ acts expansively on $X$. We have to show that $X$ is abelian. In order to prove this claim we note that $\Gamma \cong \Gamma^{\prime} \times \Gamma^{\prime \prime}$, where $\Gamma^{\prime}$ where $\Gamma^{\prime \prime}$ are subgroups of $\Gamma$, $\Gamma^{\prime} \cong \mathbb{Z}^{d}$ for some $d \geq 1$, and $\Gamma^{\prime \prime}$ is finite. Clearly $\Gamma^{\prime}$ still acts expansively on $X$, hence $\left(X, \Gamma^{\prime}\right)$ satisfies the descending chain condition (cf. Theorem 5.2), and Theorem 3.2 
allows us to find a compact (and necessarily connected) Lie group $H$ such that $X$ can be regarded as a full, shift-invariant subgroup of $H^{\mathbb{Z}^{\prime \prime}}$. An application of Corollary 4.9 allows us to find an increasing sequence $\left(H_{n}, n \geq 1\right)$ of closed, normal subgroups of $H$ with finite centres such that $H / C(H)=H_{n} / C(H)$ for each $n \geq 1$ and $\bigcup_{n} X_{n}$ is dense in $X$, where $X_{n}=X \cap H_{n}^{\mathbb{Z}{ }^{\prime \prime}}$. We fix $n \geq 1$ for the moment, denote by $Y$ the connected component of the identity in $X_{n}$, and put $G=\pi_{\{0\}}(Y) \subset H_{n}$. Since $X$ is full in $H^{\mathbb{Z}^{\prime \prime}}$ and $Y$ is normal in $X, G$ is a closed, connected, normal subgroup of $H$ and hence of $H_{n}$. In particular the centre of $G$ is finite, $G^{\prime}=G / C(G)$ is connected and has trivial centre, and $C(G) \subset C(H)$. We write $\eta: G \rightarrow G^{\prime}$ for the quotient map, define the homomorphism $\boldsymbol{\eta}: G^{\mathbb{Z}^{d}} \rightarrow G^{\prime \mathbb{Z}^{\prime \prime}}$ by $\boldsymbol{\eta}(x)(\boldsymbol{m})=\eta(x(\boldsymbol{m})), \boldsymbol{m} \in \mathbb{Z}^{d}, x \in G^{\mathbb{Z}^{\prime \prime}}$, and conclude from Lemma 9.2 that $\eta(Y) \subset G^{\prime \mathbb{Z}^{d}}$ is a connected, expansive, full, shift-invariant subgroup of $G^{\prime \mathbb{Z}^{d}}$. Lemma 9.8 shows that $\boldsymbol{\eta}(Y)=\{1\}$, i.e. that $Y \subset$ $C(G)^{\mathbb{Z}^{d}} \cap C(X)$. Furthermore $X_{n} / Y$ is a zero dimensional, closed, normal subgroup of the connected group $X / Y$, and we conclude that $X_{n} / Y \subset C(X / Y)=C(X) / Y$ and therefore $X_{n} \subset C(X)$. Hence $X$ is abelian, since $\bigcup_{n} X_{n}$ is dense in $X$. This completes the proof of Theorem 7.3.

\section{The proof of Theorem 7.5}

LEMMA 10.1. Let $G$ be a compact, connected Lie group, $d \geq 1$, and let $X \subset G^{\mathbb{Z}^{\prime \prime}}$ be a full, shift-invariant subgroup. If $n \in \mathbb{Z}^{d}$ and $T_{n}$ is ergodic on $X$, then the set of $T_{n}$-periodic points is dense in $X$.

Proof. We put $G^{\prime}=G / C(G)$, write $\kappa: G \rightarrow G^{\prime}$ for the quotient map, and define a homomorphism $\kappa: G^{\mathbb{Z}^{\prime \prime}} \rightarrow G^{\mathbb{Z}^{\prime \prime}}$ by $\boldsymbol{\kappa}(x)(\boldsymbol{m})=\kappa(x(\boldsymbol{m})), \boldsymbol{m} \in \mathbb{Z}^{d}, x \in G^{\mathbb{Z}^{\prime}}$. According to Corollary 3.11 we may assume without loss in generality that

$$
X=\left\{x \in G^{\mathbb{Z}^{d}}: \pi_{I(d)}\left(T_{m} x\right) \in \pi_{I(d)}(X) \text { for every } m \in \mathbb{Z}^{d}\right\}
$$

and

$$
X^{\prime}=\boldsymbol{\kappa}(X)=\left\{x \in G^{\prime \mathbb{Z}^{\prime}}: \pi_{I(d)}\left(T_{m} x\right) \in \pi_{I(d)}\left(X^{\prime}\right) \text { for every } \boldsymbol{m} \in \mathbb{Z}^{d}\right\},
$$

where $I(d)=\{0,1\}^{d} \subset \mathbb{Z}^{d}$. We fix $n=\left(n_{1}, \ldots, n_{d}\right) \in \mathbb{Z}^{d}$ such that $T_{n}$ is ergodic on $X$, denote by $n^{\prime}=\left(n_{1}^{\prime}, \ldots, n_{d}^{\prime}\right) \in \mathbb{Z}^{d}$ the primitive element with $n=l \boldsymbol{n}^{\prime}$ for some $l \geq 1$, and choose a group $\Delta \cong \mathbb{Z}^{d-1}$ in $\mathbb{Z}^{d}$ such that $\left\{\boldsymbol{m}+k \boldsymbol{n}^{\prime}: \boldsymbol{m} \in \Delta, k \in \mathbb{Z}\right\}=\mathbb{Z}^{d}$. Let $W=$ $\pi_{\Delta}(X) \subset G^{\Delta}$ and $W^{\prime}=\pi_{\Delta}\left(X^{\prime}\right) \subset G^{\prime \Delta}$. Then $W, W^{\prime}$ are connected, full, $T_{\Delta}$-invariant subgroups of $G^{\Delta}$ and $G^{\prime \Delta}$, respectively. Exactly as in the proof of Lemma 9.7 we define continuous, injective homomorphisms $\eta: X \rightarrow W^{\mathbb{Z}}, \eta^{\prime}: X^{\prime} \rightarrow W^{\prime \mathbb{Z}}$, by $\eta(x)$ $(k)=\pi_{\Delta}\left(T_{k n} x\right)$ and $\eta^{\prime}\left(x^{\prime}\right)(k)=\pi_{\Delta}\left(T_{k n} x^{\prime}\right), k \in \mathbb{Z}, x \in X, x^{\prime} \in X^{\prime}$, and observe that $\eta(X)=Y_{H}, \eta^{\prime}\left(X^{\prime}\right)=Y_{H^{\prime}}$ with $H=\pi_{\{0,1\}}(\eta(X)), H^{\prime}=\pi_{\{0,1\}}\left(\eta^{\prime}\left(X^{\prime}\right)\right)$. If $\kappa^{\prime}: G^{\Delta} \rightarrow G^{\prime \Delta}$ is the obvious quotient map arising from $\kappa: G \rightarrow G^{\prime}$, then it is clear that

$$
H^{\prime} \subset\left(\boldsymbol{\kappa}^{\prime} \times \boldsymbol{\kappa}^{\prime}\right)(H) \text {. }
$$

Consider the closed, normal, $T_{\Delta}$-invariant subgroups $F_{H}(n) \subset W, F_{H^{\prime}}(n) \subset W^{\prime}$, $n \in \mathbb{Z}$, defined by (5.4). From (10.3) we know that $F_{H^{\prime}}(n) \subset \kappa^{\prime}\left(F_{H}(n)\right)$ for every $n \in \mathbb{Z}$. As in the proof of Lemma 9.7 we conclude that there exists an integer $K \geq 1$ such that

$$
\boldsymbol{\kappa}^{\prime}\left(F_{H}(K)\right)=F_{H^{\prime}}(K)=W^{\prime},
$$


and we conclude that $V=W / F_{H}(K)$ is abelian. Put $Y_{K}=Y_{H} \cap F_{H}(K)^{\mathbb{Z}}$ and apply Lemma 8.3 to see that the shift $T=T_{1}$ is ergodic on $Y_{K}$, that the set of shift-periodic points is dense in $Y_{K}$, and that there exists, for every $y \in Y_{K}$ and every $m \geq 1$, a point $v \in Y_{K}$ such that $y=\left(T_{m} v\right) v^{-1}$. We write $\theta: W \rightarrow V$ for the quotient map and define a homomorphism $\theta: Y_{H} \rightarrow V^{\mathbb{Z}}$ by setting $\theta(u)(n)=\theta(u(n))$ for every $u \in \eta(X)$. Then $\operatorname{ker}(\boldsymbol{\theta})=Y_{K}$, and $\boldsymbol{\theta}\left(Y_{H}\right) \subset V^{\mathbb{Z}}$ is a closed, ergodic, shift invariant, abelian subgroup.

The group $U=\operatorname{ker}(\theta \cdot \eta) \subset X$ is closed, normal, and shift invariant, $X / U$ is abelian, $T_{n}$ is ergodic on $U$, and the set of $T_{n^{\prime}}$-periodic points is dense in $U$. Furthermore there exists, for every $u \in U$ and $m \geq 1$, a point $v \in U$ such that $y=\left(T_{m n} \cdot v\right) v^{-1}$. From Theorem 7.2 we know that the set of shift-periodic (and hence the set of $T_{n}$-periodic) points is dense is $X / U$, and Lemma 8.1 implies that the set of $T_{n}$-periodic points is dense in $X$.

Proof of Theorem 7.5. Since $(X, \Gamma)$ satisfies the descending chain condition we may assume that $X$ is a full, shift-invariant subgroup of $H^{\Gamma}$, where $H$ is a compact Lie group, and the last part in the proof of Theorem 3.2 allows us to assume that $\Gamma=\mathbb{Z}^{d}$ for some $d \geq 1$. Suppose that $T_{n}$ is ergodic on $X$ for some $n \in \mathbb{Z}^{d}$. By Corollary 5.10 the restriction of $T_{n}$ to $X^{0}$ is ergodic, where $X^{0}$ is the connected component of the identity in $X$. We write $H^{0}$ for the connected component of the identity in $H$ and note that $X^{0}$ is a full, shift invariant subgroup of $\left(H^{0}\right)^{\mathbb{Z}^{d}}$. Theorem 7.2 shows that the set of shift-periodic points (and hence the set of $T_{n}$-periodic points) is dense in $X / X^{0}$, Lemma 10.1 implies that the $T_{n}$-periodic points are dense in $X^{0}$, and Proposition 5.9 yields the density of the set of $T_{n}$-periodic points in $X$.

\section{Commuting automorphisms of compact, abelian groups}

In this section we give a brief description of pairs $(X, \Gamma)$, where $X$ is a compact, abelian group and $\Gamma \subset$ Aut $(X)$ a finitely generated, abelian group. As we have seen in the last part of the proof of Theorem 3.2, it is no real restriction to assume that $\Gamma \cong \mathbb{Z}^{d}$ for some $d \geq 1$, and we shall do so throughout the following discussion. Consider the ring

$$
\boldsymbol{R}=\boldsymbol{R}(d)=\mathbb{Z}\left[u_{1}, \ldots, u_{d}, u_{1}^{-1}, \ldots, u_{d}^{-1}\right]
$$

of all Laurent polynomials in the variables $u_{1}, \ldots, u_{d}$ with coefficients in $\mathbb{Z}$. If $\mathcal{M}$ is a finitely generated $\boldsymbol{R}$-module and $\left\{f_{1}, \ldots, f_{n}\right\} \subset \mathcal{M}$ we write $\left\langle f_{1}, \ldots, f_{n}\right\rangle=$ $\boldsymbol{R} f_{1}+\cdots+\boldsymbol{R} f_{n}$ for the submodule of $\mathcal{M}$ generated by $\left\{f_{1}, \ldots, f_{n}\right\}$. Let $X=X^{\mathscr{\mu}}=\mathscr{M}^{\hat{\imath}}$ be the dual group of $\mathcal{M}$, where $\mathcal{M}$ is regarded as a countable, discrete, abelian group under addition. Then $\mathbb{Z}^{d}$ acts on $X^{\prime \prime}$ in a natural way as a group of automorphisms. In order to describe this action we denote a typical element of $\mathbb{Z}^{d}$ by $n=\left(n_{1}, \ldots, n_{d}\right)$ and put $u^{n}=u_{1}^{n_{1}} \cdots \cdots u_{d}^{n^{d}}$. The identity element in $\mathbb{Z}^{d}$ will be denoted by 0 . Every $\mathbf{n} \in \mathbb{Z}^{\mathrm{d}}$ defines an automorphism $\beta_{n}$ of $\mathcal{M}$ by

$$
\beta_{n}(f)=\beta_{n}^{\prime \prime}(f)=u^{n} \cdot f, \quad f \in \mathcal{M},
$$

and we set

$$
\alpha_{n}=\alpha_{n}^{u n}=\hat{\beta_{n}}
$$


where $\hat{\beta_{n}}$ is the automorphism of $X^{\cdot \mu}$ dual to $\beta_{n}$, and

$$
\Gamma=\Gamma^{\prime \prime}=\left\{\alpha_{n}^{\prime \prime}: n \in \mathbb{Z}^{d}\right\} \subset \operatorname{Aut}\left(X^{\prime \prime}\right) .
$$

Lemma 11.1. Assume that $d \geq 1, \mathcal{M}=\boldsymbol{R}(d)$, and let $X=\boldsymbol{R}(d) \hat{)}$. Then there exists a continuous isomorphism $\varphi: X \rightarrow \mathbb{J}^{\mathbb{Z}^{\prime}}$ such that $\varphi \cdot \alpha_{n}=T_{n} \cdot \varphi$ for every $n \in \mathbb{Z}^{d}$, where $T_{n}$ denotes the shift by $n$ on $\mathbb{T}^{\mathbb{Z}^{\prime \prime}}($ cf. (2.3) and (11.3)). In particular $(X, \Gamma)$ satisfies the descending chain condition, where $\Gamma$ is defined in (11.4).

Proof. From (11.1) we obtain an isomorphism $\Xi: \sum_{\mathbb{Z}^{\prime \prime}} \mathbb{Z} \rightarrow \boldsymbol{R}(d)$, where $\sum_{\mathbb{Z}^{d}} \mathbb{Z}$ is the direct sum of copies of $\mathbb{Z}$ indexed by $\mathbb{Z}^{d}$ and where

$$
\Xi(c)=\sum_{n \in \mathbb{Z}^{\prime \prime}} c(\boldsymbol{n}) u^{n} \in \boldsymbol{R}(d)
$$

for every $c=\left\{c(\boldsymbol{n}): \boldsymbol{n} \in \mathbb{Z}^{d}\right\} \in \sum_{\mathbb{Z}^{\prime}} \mathbb{Z}$. For every $\boldsymbol{m} \in \mathbb{Z}^{d}$ we write $S_{\boldsymbol{m}}$ for the shift on $\sum_{\mathbb{Z}^{d}} \mathbb{Z}$ defined by

$$
S_{\boldsymbol{m}}(c)(\boldsymbol{n})=\boldsymbol{c}(\boldsymbol{n}-\boldsymbol{m}), \quad \boldsymbol{m}, \boldsymbol{n} \in \mathbb{Z}^{d},
$$

and define $\beta_{m}$ by (11.2) (for $\mathcal{M}=\boldsymbol{R}(d)$ ). Then $\Xi \cdot S_{m}=\beta_{m} \cdot \Xi$ for every $m \in \mathbb{Z}^{d}$. Let $X=\prod_{n \in \mathbb{Z}^{\prime}} \mathbb{J}$ denote the cartesian product of copies of $\mathbb{T}=\mathbb{R} / \mathbb{Z}$ indexed by $\mathbb{Z}^{d}$. For every $c=\left\{c(n): n \in \mathbb{Z}^{d}\right\} \in \sum_{\mathbb{Z}^{d}} \mathbb{Z}$ we obtain a continuous homomorphism $\chi_{c}: X \rightarrow \mathbb{T}$ by

$$
X_{c}(x)=\sum_{n \in \mathbb{Z}^{\prime}} c(\boldsymbol{n}) x(\boldsymbol{n}),
$$

where

$$
x=\left(x(n): n \in \mathbb{Z}^{d}\right) \in X .
$$

Equation (11.7) gives a specific isomorphism $\eta: \sum_{\mathbb{Z}^{d}} \mathbb{T} \rightarrow X^{\wedge}$, and the isomorphism

$$
\varphi: X \rightarrow \boldsymbol{R}(d) \hat{~}
$$

dual to $\eta \cdot \Xi^{-1}: \boldsymbol{R}(d) \rightarrow X^{\wedge}$ allows us to identify $X$ with $\boldsymbol{R}(d) \hat{\wedge}$. Clearly, the automorphisms $T_{m}=\left(S_{m}\right)^{\wedge}$ and $\alpha_{m}=\left(\beta_{m}\right)^{\wedge}$ dual to $S_{m}$ and $\beta_{m}$ correspond under $\varphi$, i.e. $\varphi \cdot \alpha_{m}=T_{m} \cdot \varphi$. The last assertion follows from Theorem 3.2.

THEOREM 11.2. Let $d \geq 1, \mathcal{M}$ is a finitely generated $\boldsymbol{R}(d)$-module, and put $X=X^{\prime \prime}=$ $\hat{M}$. If $\Gamma=\left\{\alpha_{n}: n \in \mathbb{Z}^{d}\right\} \subset$ Aut $(X)$ is the group defined by (11.2)-(11.4), then the following is true.

(1) The pair $(X, \Gamma)$ satisfies the descending chain condition.

(2) There exists an integer $k \geq 1$ and a continuous, injective homomorphism $\varphi: X \rightarrow\left(\mathbb{T}^{k}\right)^{\mathbb{Z}^{\prime}}$ such that

$$
\varphi \cdot \alpha_{n}=T_{n} \cdot \varphi
$$

for every $n \in \mathbb{Z}^{d}$, i.e. $\varphi$ is a conjugacy of $(X, \Gamma)$ into $\left(\mathbb{T}^{k}\right)^{\mathbb{Z}^{d}}$.

(3) The closed, $\Gamma$-invariant subgroups of $X$ (or, equivalently, the closed, shiftinvariant subgroups of $\varphi(X) \subset\left(\mathbb{Z}^{k}\right)^{\mathbb{Z}^{d}}$ ) are in one-to-one correspondence with the submodules of $\mathcal{M}$ : if $V$ is a closed, $\Gamma$-invariant subgroup of $X$ and $V^{\perp} \subset \mathcal{M}=$ $X^{\wedge}$ is the annihilator subgroup of $V$ in $\mathcal{M}$, then $V^{\perp}$ is a submodule of $\mathcal{M}$; conversely, if $\mathcal{N} \subset \mathcal{M}$ is a submodule, then $\mathcal{N}^{\perp}$ is a closed, $\Gamma$-invariant subgroup of $X$.

(4) For every $n \in \mathbb{Z}^{d}$, the automorphism $\alpha_{n}$ is ergodic on $X$ if and only if, for every $l \geq 1$, multiplication by the polynomial $\left(u^{\prime \prime}-1\right)$ on $\mathcal{M}$ is injective. If $\alpha_{n}$ is ergodic on $X$ for every $\mathbf{0} \neq n \in \mathbb{Z}^{d}$ then the action of $\Gamma$ on $X$ is mixing. 
Proof. This follows from Lemma 11.1, Theorem 2.4, Lemma 3.3, and duality considerations.

Remark 11.3. For a compact group $X$ of the form $X=\mu^{\wedge}$, the descending chain condition on $(X, \Gamma)$ is equivalent to the ascending chain (or Noetherian) condition on submodules of $\mathcal{M}$.

THEOREM 11.4. Let $Y$ be a compact, abelian group, and let $\Delta \subset \operatorname{Aut}(Y)$ be a finitely generated, abelian group such that $(Y, \Delta)$ satisfies the descending chain condition. Then there exists an integer $d \geq 1$, a finitely generated $\boldsymbol{R}(d)$-module $\mathcal{M}$, a continuous isomorphism $\psi: X^{\cdot t h} \rightarrow Y$, and a surjective homomorphism $\zeta: \mathbb{Z}^{d} \rightarrow \Delta$ such that $\psi\left(\alpha_{n}^{\prime \prime \prime}(x)\right)=\zeta(n)(\psi(x))$ for every $x \in X^{\prime \prime}$ and $n \in \mathbb{Z}^{d}$, where $\Gamma^{\text {" }}=$ $\left\{\alpha_{n}^{\prime \prime}: n \in \mathbb{Z}^{d}\right\} \subset$ Aut $\left(X^{\prime \prime}\right)$ is defined in (11.2)-(11.4).

Proof. Since $(Y, \Delta)$ satisfies the descending chain condition we may assume without loss in generality that $Y$ is a closed, shift-invariant subgroup of $\left(\mathbb{T}^{k}\right)^{\Delta}$ for some $k \geq 1$ (cf. Theorem 3.2). Furthermore there exists a $d \geq 1$ and a surjective homomorphism $\zeta: \mathbb{Z}^{d} \rightarrow \Delta$, and we define a continuous, injective homomorphism $\xi: Y \rightarrow\left(\mathbb{T}^{k}\right)^{\mathbb{Z}^{d}}$ by setting $\xi(y)(\boldsymbol{n})=y(\zeta(\boldsymbol{n}))$ for every $y \in Y, \boldsymbol{n} \in \mathbb{Z}^{d}$. Note that $\xi \cdot T_{\zeta(n)}^{\prime}=T_{n} \cdot \xi$ for all $n \in \mathbb{Z}^{d}$, where $T_{\perp}^{\prime}$ and $T_{\mathbb{Z}^{\prime}}$ denote the shifts on $\left(\mathbb{T}^{k}\right)^{\Delta}$ and on $\left(\mathbb{T}^{k}\right)^{\mathbb{Z}^{d}}$, respectively. Theorem 11.2(2) yields an isomorphism $\varphi: X=\left(\boldsymbol{R}(d)^{k}\right)^{\wedge} \rightarrow$ $\left(\mathbb{T}^{k}\right)^{\mathbb{Z}^{d}}$ satisfying (11.9). By Theorem 11.2(3) there exists a submodule $\mathcal{N} \subset \boldsymbol{R}(d)^{k}$ with $\varphi^{-1}(\xi(Y))=\left(\boldsymbol{R}(d)^{k} / \mathcal{N}\right)^{\hat{n}}$, and the proof is completed by setting $\mathscr{M}=\boldsymbol{R}(d)^{k} / \mathcal{N}$ and $\psi=\xi^{-1} \cdot \varphi: X^{\cdot t} \rightarrow Y$.

COROLlary 11.5. Let $Y$ be a compact, abelian group, let $\Gamma \subset \operatorname{Aut}(Y)$ be isomorphic to $\mathbb{Z}^{d}$, and assume that $(Y, \Gamma)$ satisfies the descending chain condition. Since $\Gamma \cong \mathbb{Z}^{d}$, we can write it in the form $\Gamma=\left\{\gamma_{n}: n \in \mathbb{Z}^{d}\right\}$. Then there exists an integer $k \geq 1$, a finitely generated $\boldsymbol{R}(d)$-module $\mathcal{M}$, and a continuous isomorphism $\psi: X^{\prime \prime \prime} \rightarrow Y$ such that $\psi\left(\alpha_{n}^{\prime \prime}(x)\right)=\gamma_{n} \psi(x)$ for every $n \in \mathbb{Z}^{d}, x \in X^{\prime \prime \prime}$.

We are grateful to $\mathrm{B}$. Hartley for communicating to us the following simple proof of Theorem 7.2 under the additional assumption that $Y$ is abelian.

THEOREM 11.6. Let $Y$ be a compact, abelian group, and let $\Gamma \subset$ Aut $(Y)$ be a finitely generated, abelian group such that $(Y, \Gamma)$ satisfies the descending chain condition. Then the set of $\Gamma$-periodic points is dense in $Y$.

Proof. By Theorem 11.4 we may assume that $\Gamma=\mathbb{Z}^{d}$ for some $d \geq 1$, and that there exists a finitely generated $\boldsymbol{R}(d)$-module $\mathcal{M}$ such that $X=\mathcal{M}^{\hat{n}}$ and $\Gamma=\Gamma^{\mu}=$ $\left\{\alpha_{n}^{\mathscr{M}}: n \in \mathbb{Z}^{d}\right\}$. Fix $f \neq 0$ in $\mathcal{M}$ and choose a submodule $\mathcal{M}_{f} \subset \mathcal{M}$ which is maximal with respect to the property that $f \notin \mathscr{M}_{f}$. Then the $\boldsymbol{R}(d)$-module $\mathscr{M}^{\prime}=\mathscr{M} / \mathscr{M}_{f}$ has the minimial nonzero submodule $\mathscr{M}_{1}=\left(\langle f\rangle+\mathscr{M}_{f}\right) / \mathscr{M}_{f}$.

We claim that $\mathcal{M}^{\prime}$ is finite. Indeed, if $\mathscr{J} \subset \boldsymbol{R}(D)$ is defined by $\mathscr{J}=$ $\left\{h \in \boldsymbol{R}(d): h \mathcal{M}_{1}=\{0\}\right\}$, then $\mathscr{J}$ is a maximal ideal (cf. Corollary 2.5 in [AM]), and $k=\boldsymbol{R}(d) / \mathscr{J}$ is a field which is a finite $\boldsymbol{R}(d)$-module and hence finite. The Artin-Rees lemma (Corollary 10.10 in [AM]) implies that there exists an integer $m \geq 1$ such that $\mathscr{J}^{m} \mathcal{M}^{\prime} \subset \mathscr{I} \mathscr{M}_{1}=\{0\}$. Since $k=\boldsymbol{R}(d) / \mathscr{J}$ is finite, the decreasing sequence of $\boldsymbol{R}(d)$-modules $\mathscr{M}^{\prime} \supset \mathscr{J} \mathcal{M}^{\prime} \supset \mathscr{J}^{2} \mathcal{M}^{\prime} \supset \cdots \supset \mathscr{J}^{m} \mathcal{M}^{\prime}=\{0\}$ has the property that the quotients $\mathscr{J}^{r} \mathcal{M}^{\prime} / \mathscr{J}^{r+1} \mathcal{M}^{\prime}, 0 \leq r<m$, are all finite, and hence $\mathcal{M}^{\prime}$ is finite, as claimed. 
We have thus found, for every nonzero $f \in \mathcal{M}=\hat{Y}$, a submodule $\mathcal{M}_{f} \subset \mathcal{M}$ such that $f \notin \mathscr{M}_{f}$ and $\mathcal{M} / \mathcal{M}_{f}$ is finite. The subgroup $Y_{f}=\mathscr{M}_{f}^{\perp} \subset Y$ is therefore finite, and is not annihilated by $f$. In particular there exists a $\Gamma$-periodic point $y \in Y_{f}$ which is not annihilated by the character $f$ of $Y$. It follows that the group $\operatorname{Per}(\Gamma)$ of $\Gamma$-periodic points is dense in $Y$.

Remark 11.7. From an algebraic point of view it would have been more natural to put $\boldsymbol{R}=\boldsymbol{R}(d)=\mathbb{Z}\left[u_{1}, \ldots, u_{d}\right]$ instead of (11.2). In this case the maps $\beta_{n}: \mathcal{M} \rightarrow \mathcal{M}$ and $\alpha_{n}: X \rightarrow X, \mathbf{0} \leq n \in \mathbb{Z}^{d}$ (i.e. $n=\left(n_{1}, \ldots, n_{d}\right)$ and $n_{i} \geq 0$ for $i=1, \ldots, d$ ) defined by (11.5) and (11.6), would have been injective and surjective homomorphisms, respectively. Replacing the ring $\mathbb{Z}\left[u_{1}, \ldots, u_{d}\right]$ by its localization $\boldsymbol{R}(d)$ is equivalent to replacing the surjective homomorphisms $\alpha_{n}, 0 \leq n \in \mathbb{Z}^{d}$, by their natural extensions to automorphisms.

\section{Examples of commuting automorphisms of compact, abelian groups}

As we have seen in $\S 8$, there exists a very simple algebraic formalism for describing all pairs $(X, \Gamma)$, where $X$ is a compact, abelian group and $\Gamma \subset \operatorname{Aut}(X)$ a finitely generated, abelian group such that $(X, \Gamma)$ satisfies the descending chain condition. In this section we give some examples of dynamical systems of this form. A more detailed study of dynamical properties of such systems will appear in a subsequent paper.

Let $d \geq 1$ be fixed, put $R=R(d)$ (cf. (11.2)), and let $\mathcal{M}$ be an $R$-module. We put $X=\mathcal{M}^{\wedge}$ and define the group $\Gamma=\left\{\alpha_{n}: n \in \mathbb{Z}^{d}\right\}$ as in (11.2)-(11.4). The conjugacy $\varphi$ of $(X, \Gamma)$ into $\left(\mathbb{T}^{k}\right)^{\mathbb{Z}^{d}}$ (cf. theorem $\left.11.2(2)\right)$ allows us to identify $X$ with a closed, shift-invariant subgroup of $\left(\mathbb{T}^{k}\right)^{\mathbb{Z}^{\prime \prime}}$ and to assume that $\Gamma$ is the group of shifts on $X$. If the module $\mathcal{M}$ is of the form $\mathcal{M}=\boldsymbol{R} /\langle f\rangle=\boldsymbol{R} / \boldsymbol{R} \cdot f$ we shall write $X^{f}, \alpha_{n}^{f}$ and $\Gamma^{f}$ instead of $X^{R /\langle f\rangle}, \alpha_{n}^{R /\langle f\rangle}$ and $\Gamma^{R /\langle f\rangle}$.

Examples 12.1. Let $d=1$ and $\mathcal{M}=\boldsymbol{R} /\langle f\rangle$ for some $f \in \boldsymbol{R}=\boldsymbol{R}(1)$.

(1) Let $a, b \in \mathbb{Z}$ be relatively prime. If $f(u)=a-b u$, then $X^{f}=Y_{H(A)}$, where $A=(a / b) \in G L(1, \mathbb{Q})$, and $\alpha_{1}^{f}=T_{(a / b)}$ (cf. Theorem 6.1 and Proposition 6.2).

(2) Let $f(u)=2-2 u$. Then there exists a homeomorphism $\psi: X^{f} \rightarrow \mathbb{T} \times \mathbb{Z}_{/ 2}^{\mathbb{Z}}$ which sends $\alpha_{1}^{f}$ to the automorphism id $\times T$ of $\mathbb{T} \times \mathbb{Z}_{/ 2}^{\mathbb{Z}}$, where $T$ is the shift on $\mathbb{Z}_{/ 2}^{\mathbb{Z}}$. In particular, $\alpha_{1}^{f}$ is nonergodic.

(3) Let $f(u) \in \boldsymbol{R}$ be arbitrary. We can multiply $f$ by one of the units $\left\{u^{k}: k \in \mathbb{Z}\right\} \subset \boldsymbol{R}$ and assume that $f(u)=c_{0}+c_{1} u+\cdots+c_{n} u^{n}$ with $c_{0} \cdot c_{n} \neq 0$. If $D$ is the highest common factor of $c_{0}, \ldots, c_{n}$, then there exists a homeomorphism $\psi: X^{f} \rightarrow$ $(\mathbb{Z} / D \mathbb{Z})^{\mathbb{Z}} \times X^{f / D}$ which carries $\alpha_{1}^{f}$ to $T \times \alpha_{1}^{f / D}$, where $T$ is the shift on $(\mathbb{Z} / D \mathbb{Z})^{\mathbb{Z}}$. The group $X^{f / D}$ is isomorphic to $Y_{H(A)}$, where $A^{\prime} \in G L(n, \mathbb{Q})$ is the companion matrix of the polynomial $g(u)=c_{0}^{\prime}+c_{1}^{\prime} u+\cdots+u^{n}$ with $c_{i}^{\prime}=c_{i} / c_{n}$, and $Y_{H(A)}$ is defined in (6.1) and (6.2). Clearly $\alpha_{1}^{f / D}$ is conjugate to the shift $T_{A}$ on $Y_{H(A)}$. By using Yuzvinskii's formula (6.6) we can compute the entropy of the automorphism $T_{A}$ and conclude that

$$
h\left(\alpha_{1}^{f}\right)=\log D+h\left(\alpha_{1}^{f / D}\right)=\log c_{n}+\sum_{\mid \lambda(i)>>1} \log |\lambda(i)|,
$$

where $\{\lambda(1), \ldots, \lambda(n)\}$ are the roots of the polynomial $f$. 
Examples 12.2. For the following examples we assume that $d=2$ and $\boldsymbol{R}=\boldsymbol{R}(2)$.

(1) Let $f\left(u_{1}, u_{2}\right)=1+u_{1}+u_{2}$. One can easily see that the polynomial $f$ is prime and not cyclotomic in any of the $u^{n}, n \in \mathbb{Z}^{2}$. Hence $\alpha_{n}^{f}$ is ergodic on $X^{f}$ for every nonzero $n \in \mathbb{Z}^{2}$, and the action of $\Gamma^{f}$ on $X^{f}$ is mixing (cf. Theorem 11.2(4)). Furthermore, $X^{f}=\left\{x=\left(x_{i, j}\right) \in \mathbb{T}^{Z^{2}}: x_{i, j}+x_{i, j+1}+x_{i+1, j}=0(\bmod 1)\right.$ for all $\left.i, j \in \mathbb{Z}\right\}$.

(2) Let $f\left(u_{1}, u_{2}\right)=1+u_{1}+u_{2}+u_{1} u_{2}$. The automorphisms $\alpha_{(1,0)}^{f}$ and $\alpha_{(0,1)}^{f}$ are both nonergodic, but $\alpha_{(1,1)}^{f}$ is ergodic by Theorem 11.2(4). The group $X^{f}$ is given by

$$
\left\{x=\left(x_{i, j}\right) \in X: x_{i, j}+x_{i, j+1}+x_{i+1, j}+x_{i+1, j+1}=0(\bmod 1) \text { for all } i, j \in \mathbb{Z}\right\} .
$$

(3) Let $f\left(u_{1}, u_{2}\right)=\left(2-3 u_{1}\right)\left(5-7 u_{2}\right)$. The polynomial $f$ is not prime, but $\Gamma^{f}$ is mixing on $X^{f}$ by Theorem 11.2(4). Furthermore, $X^{f}=$ $\left\{x=\left(x_{i, j}\right) \in \mathbb{T}^{\mathbf{Z}^{2}}: 10 x_{i, j}-15 x_{i+1, j}-14 x_{i, j+1}+21 x_{i+1, j+1}=0(\bmod 1)\right.$ for all $\left.i, j \in \mathbb{Z}\right\}$.

(4) Let $f_{1}\left(u_{1}, u_{2}\right)=2-3 u_{1}, f_{2}\left(u_{1}, u_{2}\right)=5-7 u_{2}$. If $\mathscr{J}=\left\langle f_{1}, f_{2}\right\rangle \subset \boldsymbol{R}$ is the ideal generated by $f_{1}$ and $f_{2}$ then $X^{\boldsymbol{R} / \mathscr{J}}=\left\{x=\left(x_{i, j}\right) \in \mathbb{T}^{\mathbb{Z}^{2}}: 3 x_{i, j}=2 x_{i+1, j}\right.$ and $7 x_{i, j}=$ $5 x_{i, j+1}(\bmod 1)$ for all $\left.i, j \in \mathbb{Z}\right\}$. Note that $\mathscr{J}$ is a prime ideal in $\boldsymbol{R}$. Since every polynomial $f \in \mathscr{J}$ vanishes at $(2 / 3,5 / 7), \mathscr{J}$ cannot contain any cyclotomic polynomial in the variable $u^{n}$ with $0 \neq n \in \mathbb{Z}^{2}$. Since $\mathscr{J}$ is prime, Theorem 11.2(4) implies that the action of $\Gamma^{\mathscr{I}}$ on $X^{R / \mathscr{J}}$ is mixing.

(5) Let $f_{i}\left(u_{1}, u_{2}\right)=1-u_{i}, i=1,2$. If $\mathscr{J}=\left\langle f_{1}, f_{2}\right\rangle \subset \boldsymbol{R}$ is the ideal generated by $f_{1}$ and $f_{2}$ then $R / \mathscr{J} \cong \mathbb{Z}$, and $X^{R / \mathscr{I}} \cong \mathbb{T}$, and the action of $\Gamma^{R / \mathscr{I}}$ on $X^{R / \mathscr{I}}$ is trivial and hence nonergodic.

(6) Let $f\left(u_{1}, u_{2}\right)=m \geq 2$. Then $X^{f} \cong(\mathbb{Z} / m \mathbb{Z})^{\mathbf{Z}^{2}}$, and $\Gamma^{f}$ corresponds to the shifts $T_{\mathbb{Z}^{2}}$ on $(\mathbb{Z} / m \mathbb{Z})^{\mathbb{Z}^{2}}$.

(7) (Ledrappier [Le].) Let $f\left(u_{1}, u_{2}\right)=1+u_{1}+u_{1}^{-1}+u_{2}+u_{2}^{-1}$, and let $\mathscr{J}=\langle 2, f\rangle$. From Theorem 11.2(4) it is clear that $\Gamma^{R / \mathscr{J}}$ is mixing on $X^{R / \mathscr{J}}$. Furthermore,

$$
X^{R / \mathscr{J}}=\left\{x \in \mathbb{Z}_{/ 2}^{\mathbb{Z}^{2}}: x_{i-1, j}+x_{i, j-1}+x_{i, j}+x_{i+1, j}+x_{i, j+1}=0(\bmod 2) \text { for all } i, j \in \mathbb{Z}\right\} .
$$

The pair $\left(X^{R / \mathscr{J}}, \Gamma^{R / \mathscr{J}}\right)$ is an example of a mixing $\mathbb{Z}^{2}$-action which is not mixing of order 4 . In order to see this note that, for every $l \geq 1$, the polynomial

$$
f^{2^{\prime}}\left(u_{1}, u_{2}\right)=1+u_{1}^{2^{\prime}}+u_{2}^{2^{\prime}}+u_{1}^{-2^{\prime}}+u_{2}^{-2^{\prime}}
$$

lies in $\mathscr{I}$, i.e. every $x \in X^{R / \mathscr{F}}$ satisfies that

$$
x_{i-2^{\prime}, j}+x_{i, j-2^{l}}+x_{i, j}+x_{i+2^{\prime}, j}+x_{i, j+2^{\prime}}=0(\bmod 2)
$$

for every $i, j \in \mathbb{Z}, l \geq 1$. From (12.2) it is clear that $\Gamma^{R / \mathscr{J}}$ cannot be 4-mixing.

(8) (Ledrappier [Le].) A mixing action of $\mathbb{Z}^{2}$ which is not 2-mixing is obtained by setting $f\left(u_{1}, u_{2}\right)=1+u_{1}+u_{2}$ and $\mathscr{J}=\langle 2, f\rangle$. For every $x \in X^{R / g}, i, j \in \mathbb{Z}$ and $l \geq 1$,

$$
x_{i, j}+x_{i+2^{i}, j}+x_{i, j+2^{\prime}}=0(\bmod 2) \text {. }
$$

By Theorem 11.2(4) $\Gamma^{R / \mathscr{F}}$ is mixing, and (12.3) shows that $\Gamma^{R / \mathscr{J}}$ is not 2-mixing.

Examples 12.3. (1) Let $d=1$. If $f \in R$ is given by $f(u)=1-2 u$ and $\mathscr{J}=\langle 4, f\rangle$, then $f^{2}=1$ lies in $\mathscr{J}$, i.e. $\mathscr{J}=\boldsymbol{R}$. Hence $X^{R / \mathscr{J}}=\{0\}$.

(2) Let $d=2$ and $f\left(u_{1}, u_{2}\right)=1+u_{1}+2 u_{2} \in \boldsymbol{R}=\boldsymbol{R}(2)$. If $\mathscr{g}=\langle 4, f\rangle$ then

$$
X^{R / \mathscr{I}}=\left\{x \in\left(\mathbb{Z}_{/ 4}\right)^{\mathbf{Z}^{2}}: x_{i, j}+x_{i+1, j}+2 x_{i, j+1}=0(\bmod 4) \text { for all } i, j \in \mathbb{Z}\right\} \text {. }
$$

Since the polynomials $f^{2}=\left(1+u_{1}\right)^{2}$ and $2 f=2+2 u_{1}$ lie in $\mathscr{J}$ we conclude that $f^{2}-2 f=u_{1}^{2}-1 \in \mathscr{I}$, i.e. that the automorphism $\alpha_{(1,0)}^{R / \mathscr{J}}$ has period 2 and is nonergodic. 
The automorphism $\alpha_{(0,1)}^{R / \mathscr{F}}$, on the other hand, is ergodic on $X^{R / \mathscr{I}}$ and has entropy $\log 4$.

\section{REFERENCES}

[Ab] L. M. Abramov. The entropy of an automorphism of a solenoidal group. Theory Prob. Appl. 4 (1959), 231-236.

[AP] R. L. Adler \& R. Palais. Homeomorphic conjugacy of automorphisms of the torus. Proc. Amer. Math. Soc. 16 (1965), 1222-1225.

[Ao] N. Aoki. A simple proof of the Bernoullicity of ergodic automorphisms of compact abelian groups. Israel J. Math. 38 (1981), 189-198.

[AD] N. Aoki \& M. Dateyama. The relationship between algebraic numbers and expansiveness of automorphisms on compact abelian groups. Fundamenta Math. 117 (1983), 21-35.

[Ar] D. Z. Arov. The computation of the entropy for one class of group endomorphisms. Zap. Mekh.-Matem. Fakultera Kharkov Matem. 30 (1964), 48-69.

[AM] M. F. Atiyah \& I. G. MacDonald. Introduction to Commutative Algebra. Addison-Wesley: Reading, Mass., 1969.

[Be] D. Berend. Ergodic semigroups of epimorphisms. Trans. Amer. Math. Soc. 289 (1985), 393-407.

[Bo] R. Bowen. Equilibrium states and the ergodic theory of Anosov diffeomorphisms. Lecture Notes in Mathematics. 470, Springer: Berlin-Heidelberg-New York, 1975.

[Dy] H. A. Dye. On the ergodic mixing theorem. Trans. Amer. Math. Soc. 118 (1965), 123-130.

[ES] S. Eilenberg \& N. Steenrod. Foundations of Algebraic Topology. Princeton University Press: Princeton, 1952.

[Fr] D. Fried. Finitely presented dynamical systems. Ergod. Th. \& Dynam. Sys. 7 (1987), 489-507.

[GS] F. J. Grunewald \& D. Segal. Decision problems concerning S-arithmetic groups. J. Symbolic Logic 50 (1985), 743-772.

[Ha] P. R. Halmos. On automorphisms of compact groups. Bull. Amer. Math. Soc. 49 (1943), 619-624.

[Ka] I. Kaplansky. Groups with representations of bounded degree. Can. J. Math. 1 (1949), 105-112.

[Ki] B. P. Kitchens. Expansive dynamics on zero-dimensional groups. Ergod. Th. \& Dynam. Sys. 7 (1987), 249-261.

[La] P.-F. Lam. On expansive transformation groups. Trans. Amer. Math. Soc. 105 (1970), 131-138.

[Ln] W. Lawton. The structure of compact connected groups which admit an expansive automorphism. In: Recent advances in Topological Dynamics, Lecture Notes in Mathematics. Springer: BerlinHeidelberg-New York, 1973, pp. 182-196.

[LP] R. Laxton \& W. Parry. On the periodic points of certain automorphisms and a system of polynomial identities. J. Algebra 6 (1967), 388-393.

[Le] F. Ledrappier. Un champ markovien peut être d'entropie nulle et mélangeant. C.R. Acad. Sc. Paris, Ser. A. 287 (1978), 561-562.

[LSW] D. A. Lind, K. Schmidt \& T. Ward. Mahler measures and entropy for automorphisms of compact groups. In preparation.

[LW] D. A. Lind \& T. Ward. Automorphisms of solenoids and p-adic entropy. Ergod. Th. \& Dynam. Sys. 8 (1988), 411-419.

[MT] G. Miles \& R. K. Thomas. The breakdown of automorphisms of compact topological groups. In: Studies in Probability and Ergodic Theory, Advances in Mathematics Supplementary Studies, 2, Academic Press: New York-London, 1978, pp. 207-218.

[Re] W. Reddy. The existence of expansive homeomorphisms on manifolds. Duke Math. J. 32 (1965), 494-509.

[Ro] R. M. Robinson. Undecidability and nonperiodicity for tilings of the plane. Inventiones Math. 12 (1971), 177-209.

[Ru] D. Ruelle. Thermodynamic Formalism. Addison-Wesley: Reading, Mass., 1978.

[S1] K. Schmidt. Asymptotic properties of unitary representations and mixing. Proc. London Math. Soc. 48 (1984), 445-460.

[S2] K. Schmidt. Automorphisms of compact abelian groups and affine varieties. Preprint (1987).

[S3] K. Schmidt. Mixing automorphisms of compact groups and a theorem by Kurt Mahler. Pacific J. Math. (to appear).

[W1] R. F. Williams. A note on unstable homeomorphisms. Proc. Amer. Math. Soc. 6 (1955), 308-309. 
[W2] R. F. Williams. Classification of subshifts of finite type. Annals Math. 98 (1973), 120-153. Errata: 99 (1974), 380-381.

[Ws] A. M. Wilson. On endomorphisms of a solenoid. Proc. Amer. Math. Soc. 55 (1976), 69-74.

[Wu] T. S. Wu. Expansive automorphisms of compact groups. Math. Scand. 18 (1966), 13-24.

[Yu] S. A. Yuzvinskii. Computing the entropy of a group of endomorphisms. Siberian Math. J. 8 (1967), 172-178. 